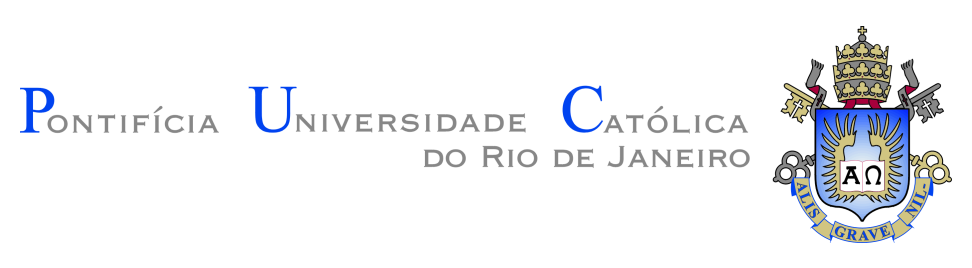

João Miranda Carnevale

\title{
Grupos de homeomorfismos da reta com um número finito de pontos fixos
}

Dissertação apresentada como requisito parcial para obtenção do grau de Mestre pelo Programa de Pós-graduação em Matemática do Departamento de Matemáticado Centro Técnico Científicoda PUC-Rio .

Orientador : Prof. Lorenzo Justiano Díaz Casado Co-orientador: Prof. Christian César Bonatti 


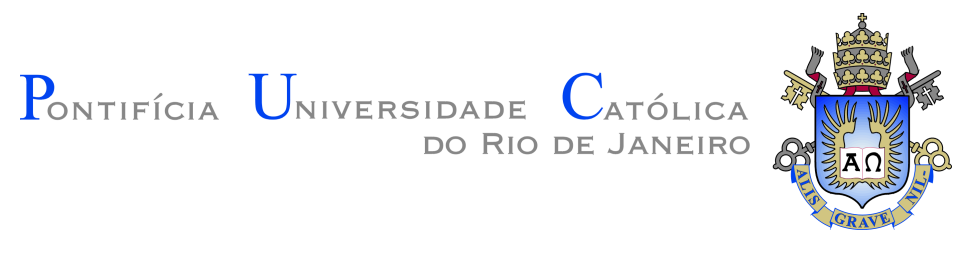

João Miranda Carnevale

\title{
Grupos de homeomorfismos da reta com um número finito de pontos fixos
}

\begin{abstract}
Dissertação apresentada como requisito parcial para obtenção do grau de Mestre pelo Programa de Pós-graduação em Matemática do Departamento de Matemáticado Centro Técnico Científicoda PUC-Rio . Aprovada pela Comissão Examinadora abaixo assinada.
\end{abstract}

Prof. Lorenzo Justiano Díaz Casado

Orientador

Pontifícia Universidade Católica do Rio de Janeiro - PUC-Rio

Prof. Christian César Bonatti

Co-orientador Université de Paris VII -

Prof. David Francisco Martínez Torres

Pontifícia Universidade Católica do Rio de Janeiro - PUC-Rio

Prof. Paul Alexander Schweitzer

Pontifícia Universidade Católica do Rio de Janeiro - PUC-Rio

Prof. Carlos Meniño Cotón

Universidade Federal Fluminense - UFF

Prof. Alejandro Kocsard

Universidade Federal Fluminense - UFF

Prof. Márcio da Silveira Carvalho

Coordenador Setorial do Centro Técnico Científico - PUC-Rio 
Todos os direitos reservados. É proibida a reprodução total ou parcial do trabalho sem autorização da universidade, do autor e do orientador.

\section{João Miranda Carnevale}

Graduou-se em Matemática pela Pontíficia Universidade Católica do Rio de Janeiro (Rio de Janeiro, Brasil).

Ficha Catalográfica

Carnevale, João Miranda

Grupos de homeomorfismos da reta com um número finito de pontos fixos / João Miranda Carnevale; orientador: Lorenzo Justiano Díaz Casado; co-orientador: Christian César Bonatti. - Rio de janeiro: PUC-Rio, Departamento de Matemática, 2018.

v., 111 f: il. color. ; $30 \mathrm{~cm}$

Dissertação (mestrado) - Pontifícia Universidade Católica do Rio de Janeiro, Departamento de Matemática.

Inclui bibliografia

1. Matemática - Teses. 2. Sistemas Dinâmicos - Teses. 3. Ação de Grupos - Teses. 4. Ação de Grupos;. 5. Teorema de Hölder;. 6. Teorema de Solodov;. 7. Abertura de Órbita;. I. Díaz Casado, Lorenzo Justiano. II. Bonatti, Christian César. III. Pontifícia Universidade Católica do Rio de Janeiro. Departamento de Matemática. IV. Título. 
Aos professores e colegas que me acompanharam durante todos os anos de estudo e diversão. 


\section{Agradecimentos}

Agradeço à Valéria, minha mãe, e ao Florindo, meu pai, pelo carinho, confiança e suporte que dedicam a mim por mais de 22 anos, ao Christian pelo acolhimento e atenção durante minha visita a Dijon, ao Lorenzo por todos os conselhos e sugestões na produção da dissertação e à CAPES por financiar minha pesquisa. 


\section{Resumo}

Carnevale, João Miranda; Díaz Casado, Lorenzo Justiano; Bonatti, Christian César. Grupos de homeomorfismos da reta com um número finito de pontos fixos. Rio de Janeiro, 2018. 111p. Dissertação de Mestrado - Departamento de Matemática, Pontifícia Universidade Católica do Rio de Janeiro.

Temos como objetivo principal classificar, a menos de semi-conjugação, os grupos de homeomorfismos crescentes da reta, com no máximo $\mathrm{N}$ pontos fixos. Começando pelos casos já classificados, $\mathrm{N}=0$ pelo Teorema de Hölder e N=1 pelo Teorema de Solodov, nosso próximo passo será classificar o caso $\mathrm{N}=2$. Para isso, iremos introduzir a noção de Abertura de Órbita como uma semi-conjugação dos grupos de homeomorfismos.

\section{Palavras-chave}

Ação de Grupos; Teorema de Hölder; Teorema de Solodov; Abertura de Órbita; 


\section{Abstract}

Carnevale, João Miranda; Díaz Casado, Lorenzo Justiano (Advisor); Bonatti, Christian César (Co-Advisor). Groups of homeomorphisms of the line with a finite number of fixed points. Rio de Janeiro, 2018. 111p. Dissertação de Mestrado - Departamento de Matemática, Pontifícia Universidade Católica do Rio de Janeiro.

We have as main objective to classify, by semi-conjugation, the groups of increasing homeomorphisms of the line, with at most $\mathrm{N}$ fixed points. Starting with the cases already classified, $\mathrm{N}=0$ by Hölder's Theorem and $\mathrm{N}=1$ by Solodov's Theorem, our next step will be to classify the case $\mathrm{N}=2$. For this, we will introduce the notion of Orbital Opening as a semiconjugation of the groups of homeomorphisms.

\section{Keywords}

Group Action; Hölder's Theorem; Solodov's Theorem; Orbital Opening; 


\section{Sumário}

1 Introdução $\quad 10$

2 Definições e primeiros resultados $\quad 14$

$\begin{array}{lll}2.1 & \text { Ordem e topologia em } \mathbb{R} & 14\end{array}$

2.2 Ação de grupos 26

2.3 Conjuntos minimais de $\mathbb{R} \quad 40$

2.3.1 Definição e classificação dos conjuntos minimais de $\mathbb{R}$

2.3.2 Critérios para existência de conjuntos minimais 45

3 Ações de grupos com até um ponto fixo 48

3.1 Ações sem pontos fixos 48

3.2 Ações com um ponto fixo global $\quad 57$

3.3 O Teorema de Solodov 58

4 Abertura de órbita $\quad 69$

4.1 Existência da abertura de uma órbita 69

$\begin{array}{lll}\text { 4.1.1 Abertura do conjunto } A & 71\end{array}$

$\begin{array}{ll}\text { 4.1.2 Extensão da ação para } \mathbb{R} & 74\end{array}$

$\begin{array}{lll}4.2 & \text { Unicidade da construção } & 78\end{array}$

4.3 Construção de grupo com no máximo N pontos fixos 82

5 Ações com no máximo dois pontos fixos $\quad 88$

5.1 Caso existe um ponto fixo global 88

$\begin{array}{ll}5.2 \text { Caso sem pontos fixos globais } & 90\end{array}$

5.2.1 Prova do Teorema 5.2.1 no caso (2) 91

5.2.2 Prova do Teorema 5.2.1 no caso (1) 92

5.2.3 Prova do Teorema 5.2.1 no caso (3) 92

5.3 Prova da Proposição 5.2.4 95

5.3.1 Prova da Proposição 5.2.4 nos casos de tangência 98

5.3.2 Prova da Proposição 5.2.4 no caso $(+,-,+) \quad 105$

$\begin{array}{ll}\text { Referências bibliográficas } & 111\end{array}$ 
If you are receptive and humble, mathematics will lead you by the hand.

Paul Dirac, The Strangest Man. 


\section{Introdução}

Sistemas dinâmicos é o campo da matemática que se dedica ao estudo do comportamento de órbitas. De forma mais precisa, dado um conjunto que interage com o tempo, estamos interessados em entender como os pontos do conjunto reagem ao passar do tempo. O primeiro exemplo é quando a ação do tempo é descrita por uma bijeção do conjunto, assim sendo $X$ o conjunto e $f$ a bijeção temos uma aplicação de $\mathbb{Z} \times X \rightarrow X$ definida por: $(x, t) \mapsto f^{t}(x)$, que descreve a posição dos pontos do conjunto para cada tempo $t$ e satisfaz $f^{r+s}(x)=f^{r} \circ f^{s}(x)$. O próximo exemplo é quando o tempo é contínuo, e a ação dele é descrita por um fluxo de funções $\phi_{t}$, nesse caso a aplicação de $\mathbb{R} \times X \rightarrow X$ definida por: $(x, t) \mapsto \phi_{t}(x)$ descreve a posição dos pontos em cada instante, e também satisfaz $\phi_{r+s}(x)=\phi_{r} \circ \phi_{s}(x)$.

Podemos, então, generalizar essa ideia e, no lugar do tempo, que é naturalmente representado pelos grupos $\mathbb{Z}$ ou $\mathbb{R}$, escolher um grupo genérico $G$. Assim, temos que uma aplicação de $G \times X \rightarrow X$ dada por $(g, x) \mapsto \psi(g)(x)$, satisfazendo $\psi\left(g_{1} g_{2}\right)(x)=\psi\left(g_{1}\right) \circ \psi\left(g_{2}\right)(x)$, define uma ação do grupo $G$ sobre o conjunto $X$. Uma outra forma de descrever essa aplicação é para cada elemento do grupo, observar a bijeção $\psi(g): X \rightarrow X$, assim obtemos uma aplicação de $G \rightarrow \operatorname{Bij}(X)$, ou se o conjunto $X$ tiver mais estrutura podemos estudar as ações contínuas e diferenciáveis, e assim seriam aplicações de $G \rightarrow \operatorname{Homeo}(X)$ ou de $G \rightarrow \operatorname{Difeo}(X)^{1}$. Tal generalização sugere uma interação entre a dinâmica da ação e a álgebra do grupo, e como veremos mais a frente, de fato existem muitas correlações entre esses dois pontos de vista.

Uma das características fundamentais de um sistema dinâmico é a existência de pontos fixos. No nosso caso, dizemos que $x \in X$ é um ponto fixo da ação de grupos se existe $g \in G$ tal que $\psi(g)(x)=x$. Além disso, $x \in X$ é um ponto fixo global da ação de grupos se para todo $g \in G$ segue que $\psi(g)(x)=x$.

Agora, dado um conjunto $X$, podemos nos perguntar quais são as possíveis ações de grupos sobre $X$, ou em outras palavras, quais são os subgrupos de $\operatorname{Bij}(X)$ ou de $\operatorname{Homeo}(X)$ ? E se colocarmos hipóteses dinâmicas,

${ }^{1} \operatorname{Denote} \operatorname{Bij}(X)$, Homeo $(X)$ e $\operatorname{Difeo}(X)$, os conjuntos de bijeções, de homeomorfismos e de difeomorfismos em $X$. 
quais grupos podem agir sem pontos fixos em $X$ ? e quais agem com até $N$ pontos fixos? Ou quais agem com um único ponto fixo global? Se $X$ for um espaço topológico, quais grupos podem ter uma ação com uma órbita densa? e uma ação com todas as órbitas densas? Estas são algumas das perguntas que consideraremos neste texto.

Para estudar tais perguntas é necessário entender o conceito de conjugação, que é muito comum em diversas áreas da matemática. Uma conjugação é, basicamente, uma mudança de variáveis. Portanto, sempre procuramos ações de grupos a menos de conjugação, ou seja, ações a menos das que são iguais após uma mudança de variáveis. Porém, em alguns casos, conjugações podem ser restritivas demais, e portanto consideraremos uma relação mais fraca que é a semi-conjugação. Ela consiste em uma "conjugação" não injetiva, ou seja, além de mudar as variáveis a semi-conjugação também colapsa alguns intervalos em pontos, perdendo uma parte da informação da ação de grupo.

Agora, estamos prontos para enunciar o objetivo principal deste trabalho. Estudaremos os grupos de homeomorfismos crescentes de $\mathbb{R}$ que agem com no máximo $N$ pontos fixos, ou seja, grupos de homeomorfismos onde cada homeomorfismo do grupo, exceto a identidade, possui no máximo $N$ pontos fixos. Buscaremos exemplos para cada $N$ e classificaremos, a menos de semiconjugação, todos os grupos de homeomorfismos crescentes de $\mathbb{R}$ que agem com no máximo dois pontos fixos.

Dois exemplos clássicos que satisfazem tal enunciado são os grupos de translações, para o caso $N=0$. E os grupos de aplicações afins, para o caso $N=1$. Porém, não existem exemplos clássicos para nenhum $N \geq 2$. Em [Ho], Hölder provou que, de fato, para $N=0$, os grupos de translações são quase o único exemplo possível, pois todos os grupos sem pontos fixos devem ser semi-conjugados a um grupo de translações. Além disso, concluiu que se o grupo age sem pontos fixos em $\mathbb{R}$, então ele é abeliano. Mostrando um exemplo fantástico, onde uma hipótese dinâmica implica numa restrição algébrica pra o grupo. Provaremos este teorema na Seção 3.1 seguindo [Bo].

Para o caso $N=1$, Solodov demonstrou que todos os grupos que agem com até um ponto fixo são semi-conjugados a um grupo de aplicações afins. Provaremos este teorema na Seção 3.3 seguindo as ideias de [Bo] e [Na].

Com esses dois casos percebemos a importância dos exemplos clássicos para esse problema, e como eles não existem para $N \geq 2$, aumenta a necessidade de construir exemplos para esses casos. Para isso, definiremos a abertura de órbita:

A abertura de órbita é um conceito equivalente a semi-conjugação, porém é construído de maneira inversa. De forma mais específica, a partir de um grupo 
$G$ agindo sobre $\mathbb{R}$, vamos construir uma nova ação que será semi-conjugada a $G$. E como a semi-conjugação colapsa alguns intervalos em pontos, a nova ação a ser construída é livre para agir de qualquer forma nesses intervalos. Portanto, podemos forçar que nossa nova ação aja com $N$ pontos fixos em cada um desses intervalos. Assim, um elemento do grupo que tinha um ponto fixo vai passar a ter novos $N$ pontos fixos após a abertura de órbita, criando uma família de exemplos de grupos que agem sobre $\mathbb{R}$ com até $N \geq 2$ pontos fixos.

Porém, todos os exemplos construídos pela abertura de órbita serão semiconjugados a grupos com no máximo um ponto fixo. O que sugere que não existem grupos com verdadeiramente $N \geq 2$ pontos fixos, apenas grupos com no máximo um ponto fixo, cuja órbita foi aberta. Em outras palavras, temos o seguinte cenário que deve ser explorado: Se um grupo age em $\mathbb{R}$ com até $N \geq 1$ pontos fixos, então ele é semi-conjugado a um grupo de aplicações afins.

Por fim, vamos mostrar que este é o cenário para o caso $N=2$. Este é um resultado original em colaboração com C. Bonatti.

Esse resultado será consequência de duas observações principais: A primeira é que se o grupo age com até $N \geq 1$ pontos fixos, então sua ação possui um conjunto minimal, isto é, um conjuto não-vazio que é fechado, invariante pela ação e que não contém nenhum subconjunto não-vazio, fechado e invariante. Assim, podemos fazer uso da classificação de conjuntos minimais de $\mathbb{R}$ e dividir o problema em 4 casos, dependendo do tipo de conjunto minimal que a ação possui, sendo os tipos: um único ponto, um conjunto discreto biilimitado, um conjunto do tipo Cantor bi-ilimitado e o conjunto $\mathbb{R}$ (ver Seção 2.3). A segunda observação é que se $N=2$, então os intervalos limitados pelos pontos fixos dos elementos são dois-a-dois disjuntos (Proposição 5.2.4). Assim, com essa observação, será possivel concluir a prova do resultado para cada um dos casos levantados pela classificação de conjuntos minimais.

Abaixo segue uma descrição mais específica daquilo que será apresentado em cada capítulo:

No capítulo 2, apresentaremos as primeiras definições e resultados gerais. Ele será dividido em três seções: ordem e topologia em $\mathbb{R}$, ação de grupos e conjuntos minimais de $\mathbb{R}$. Nas duas primeiras seções serão apresentados diversos lemas, que servirão de apoio para as provas feitas nos capítulos seguintes. Na terceira seção, vamos apresentar uma classificação para os conjuntos minimais de $\mathbb{R}$, tal classificação será essencial para o estudo dos grupos de homeomorfismos até dois pontos fixos, e no final da seção será apresentado um critério para a existência de conjuntos minimais.

No capítulo 3, vamos estudar os grupos de homeomorfismos com até um 
ponto fixo. Começando pelo Teorema de Hölder, vamos concluir que todos os grupos livres de pontos fixos são abelianos e semi-conjugados a um grupo de translações. Além disso, vamos ver que a semi-conjugação é única a menos de uma transformação afim. Depois, vamos para o Teorema de Solodov, e concluir que os grupos que agem com até um ponto fixo são semi-conjugados a um grupo de aplicações afins, e esse resultado virá da unicidade da semi-conjugação do Teorema de Hölder.

No capítulo 4, vamos apresentar o conceito de abertura de órbita. Com ele vamos enunciar um resultado que mostra uma forma de fabricar grupos com até $N$ pontos fixos para $N \geq 2$. Porém, todos os grupos criados a partir desse resultado serão semi-conjugados a um grupo com até um ponto fixo. Além disso, nenhum dos grupos com $N \geq 2$ será minimal, e nenhum com $N \geq 3$ terá órbita transitiva. Por fim, serão apresentados dois exemplos concretos de grupos obtidos a partir da abertura de órbita, incluindo um exemplo que possui uma órbita transitiva e $N=2$.

No capítulo 5, estudaremos os grupos de homeomorfismos com até dois pontos fixos, e vamos concluir que eles são semi-conjugados a um grupo de aplicações afins. Como consequência, temos que os exemplos criados a partir da abertura de órbita, descritos na seção 4.3, são de fato todos os exemplos de ação sobre $\mathbb{R}$ com até dois pontos fixos. 


\section{2 \\ Definições e primeiros resultados}

Nesse capítulo, vamos apresentar e demonstrar as primeiras definições e resultados gerais que usaremos durante o trabalho. Ele será dividido em três partes: ordem e topologia em $\mathbb{R}$, ação de grupos e conjuntos minimais de $\mathbb{R}$.

O leitor pode pular as Seções 2.1 e 2.2, voltando apenas para consultar definições ou quando algum lema for citado. A Seção 2.3 será parte essencial desse trabalho, sendo um ponto fundamental para a prova do Teorema 5.2.1, que é o principal resultado do texto.

\section{1}

\section{Ordem e topologia em $\mathbb{R}$}

A princípio, é dificil imaginar relações entre ordem, grupos e dinâmica. Porém, ordem e topologia, servem como uma ponte entre álgebra e dinâmica. Como iremos ver nos próximos capítulos, muitas vezes uma hipótese dinâmica em uma ação de grupos resulta numa relação de ordem no grupo, que se bem explorada, pode trazer conclusões espetaculares sobre a estrutura algébrica do grupo. Para isso, vamos precisar começar pelas primeiras definições:

Definição 2.1.1 Seja A um conjunto e $\preceq$ uma relação binária satisfazendo, para todo $a, b, c \in A$ :

- $a \preceq a$ (Reflexividade),

- se $a \preceq b$ e $b \preceq a$, então $a=b$ (Antissimetria),

- se $a \preceq b$ e $b \preceq c$, então $a \preceq c$ (Transitividade).

Então, $\preceq$ é uma relação de ordem parcial sobre $A$, e $(A, \preceq)$ é chamado de conjunto parcialmente ordenado.

Caso $\preceq$ também satisfaça:

- Se $a, b \in A$, então: ou $a \preceq b$, ou $b \preceq a$ (Totalidade).

Então, $\preceq$ é uma relação de ordem total sobre $A$, e $(A, \preceq)$ é chamado de conjunto totalmente ordenado.

Exemplo 2.1.2 $(\mathbb{R}, \leq)$ é um conjunto totalmente ordenado. E dado qualquer subconjunto $X \subset \mathbb{R},(X, \leq)$ também é um conjunto totalmente ordenado. 
Definição 2.1.3 Seja A um conjunto $e \prec$ uma relação binária satisfazendo, para todo $a, b, c \in A$ :

- a $\nprec$ (Irreflexividade),

- se $a \prec b$, então $b \nprec a$ (Assimetria),

- se $a \prec b$ e $b \prec c$, então $a \prec c$ (Transitividade).

Então, $\prec$ é uma relação de ordem estrita parcial sobre $A, e(A, \prec)$ é chamado de conjunto parcialmente ordenado estrito.

Caso $\prec$ também satisfaça:

- Se $a, b \in A$, então: ou $a \prec b$, ou $b \prec a$, ou $a=b$ (Totalidade).

Então, $\prec$ é uma relação de ordem estrita total sobre $A, e(A, \preceq)$ é chamado de conjunto totalmente ordenado estrito.

Além disso, dada $\preceq$ uma relação de ordem sobre $A$, a relação de ordem estrita $\prec$, fica definida como:

$-a \prec b \Leftrightarrow a \preceq b e a \neq b$.

Exemplo 2.1.4 $(\mathbb{R},<)$ é um conjunto totalmente ordenado estrito. E dado qualquer subconjunto $X \subset \mathbb{R},(X,<)$ também é um conjunto totalmente ordenado estrito.

Definição 2.1.5 Sejam $\left(A_{1}, \preceq_{1}\right)$ e $\left(A_{2}, \preceq_{2}\right)$ dois conjuntos totalmente ordenados (respectivamente estritos) e $f: A \rightarrow B$ uma aplicação satisfazendo, para todo $a, b \in A_{1}$ :

$$
a \preceq_{1} b \Rightarrow f(a) \preceq_{2} f(b) .
$$

Então, a aplicação f é dita (respectivamente estritamente) crescente.

Lema 2.1.6 Sejam $X, Y$ subconjuntos de $\mathbb{R}, h: X \rightarrow Y$ uma aplicação sobrejetiva. Então, $h$ é crescente se, e só se, para todo $a, b \in Y$, com $a<b$. Segue que,

$$
x \in h^{-1}(a), y \in h^{-1}(b) \Rightarrow x<y .
$$

Prova. Suponha $h$ crescente. Suponha por absurdo que existem $x \in h^{-1}(a)$ e $y \in h^{-1}(b)$ com $x \leq y$. Aplicando $h$, temos: $h(x) \geq h(y)$ o que implica $a \geq b$.

Suponha agora que $h$ não é crescente. Então, existem $x, y \in X$, com $x<y$ e $h(x)>h(y)$. Tome $b=h(x)$ e $a=h(y)$. Então, $y, x$ pertencem às pré-imagens de $a$ e de $b$, respectivamente, porém $y \nless x$. 
Corolário 2.1.7 Sejam $X, Y \subset \mathbb{R}$ subconjuntos e $f, g: X \rightarrow Y$ funções crescentes. Se $f^{-1} \circ g: X \rightarrow X$ está bem definida, então é crescente.

Prova. Tome $x, y \in X$, com $x<y$. A função $g$ ser crescente implica em: $g(x) \leq g(y)$. Portanto:

$$
\forall s \in f^{-1}(g(x)), \forall t \in f^{-1}(g(y)), \text { temos: } s \leq t
$$

Mas como $f^{-1} \circ g$ é uma função, $f^{-1}(g(x))$ e $f^{-1}(g(y))$ contém exatamente um elemento. Portanto: $f^{-1}(g(x)) \leq f^{-1}(g(y))$.

Definição 2.1.8 Dados $X, Y$ subconjuntos de $\mathbb{R}$, uma aplicação $f: X \rightarrow Y$ é dita contínua, se para toda sequência $\left\{x_{n}\right\}_{n \in \mathbb{N}}$ convergindo para $x_{\infty} \in X$, temos:

$$
\lim _{n \rightarrow \infty} f\left(x_{n}\right)=f\left(x_{\infty}\right)
$$

Lema 2.1.9 Sejam $[a, b] \subset \mathbb{R}$ um intervalo compacto e $f:[a, b] \rightarrow \mathbb{R}$ uma aplicação contínua. Se $f(a)$ e $f(b)$ têm sinais contrários, então existe $c \in[a, b]$ $\operatorname{com} f(c)=0$.

Prova. Suponha, $f(a)<0<f(b)$. Agora, considere o conjunto:

$$
S:=\{x \in[a, b] \mid \text { para todo } y \in[a, x] \text {, temos: } f(y) \leq 0\} .
$$

Note que o conjunto $S$ é não vazio, pois $a \in S$ e é cotado superiormente por $b$.

Portanto, $S$ possui um supremo $c \in[a, b]$. Por continuidade de $f$, temos que $f(c) \leq 0$. Porém, caso $f(c)<0$, por continuidade, existe $d>c$ com $f(d)<0$, o que é um absurdo, pois $d \in S$ e $d$ maior que o supremo do conjunto.

Logo, $f(c)=0$. O caso $f(a)>0>f(b)$ é análogo.

Definição 2.1.10 Seja $f: \mathbb{R} \rightarrow \mathbb{R}$ uma aplicação contínua, tal que existem $x_{0}, x_{1}, \ldots, x_{n} \in \mathbb{R}$ com $x_{0}<x_{1}<\ldots<x_{n}$ e $f\left(x_{k}\right), f\left(x_{k+1}\right)$ têm sinais contrários para todo $k \in\{0,1, \ldots, n-1\}$. Então, dizemos que $f$ troca de sinal pelo menos $n$ vezes.

Observação 2.1.11 Pelo Lema 2.1.9, se $f$ troca de sinal $n$ vezes, então $f$ tem no mínimo $n$ zeros.

Definição 2.1.12 Dados $X, Y$ subconjuntos de $\mathbb{R}$, uma aplicação $f: X \rightarrow Y$ é dita um homeomorfismo, se $f$ for contínua e bijetiva, com $f^{-1}$ também contínua. Nesse caso, $X, Y$ são ditos homeomorfos. 
Lema 2.1.13 Sejam $X, Y \subset \mathbb{R}$ subconjuntos fechados e $f: X \rightarrow Y$ bijetiva e crescente, então f é homeomorfismo.

Prova. A prova do lema será consequência das seguintes afirmações:

Afirmação. Seja $\left\{x_{n}\right\}_{n \in \mathbb{N}} \subset X$ uma sequência monótona com $\lim x_{n}=x$. Então,

$$
\lim _{n \rightarrow+\infty} f\left(x_{n}\right)=f(x)
$$

Prova. Suponha, $\left\{x_{n}\right\}_{n \in \mathbb{N}}$ crescente. Temos assim, para todo $n \in \mathbb{N}$ :

$$
x_{n} \leq x_{n+1} \leq x
$$

E portanto, como $f$ é crescente:

$$
f\left(x_{n}\right) \leq f\left(x_{n+1}\right) \leq f(x)
$$

Portanto, $\left\{f\left(x_{n}\right)\right\}_{n \in \mathbb{N}}$ é sequência crescente e limitada superiormente, logo, convergente. E como $Y$ é fechado, temos que existe $y \in Y$ com: $\lim _{n \rightarrow \infty} f\left(x_{n}\right)=y$. É claro que $y \leq f(x)$, e caso $y<f(x)$ temos:

$$
f^{-1}(y)<f^{-1}(f(x))=x
$$

Portanto, temos para todo $n$ :

$$
x_{n}=f^{-1}\left(f\left(x_{n}\right)\right) \leq f^{-1}(y)<x .
$$

Assim, $\lim _{n \rightarrow \infty} x_{n} \leq f^{-1}(y)<x$. Absurdo, pois $\lim _{n \rightarrow \infty} x_{n}=x$.

E o caso $\left\{x_{n}\right\}_{n \in \mathbb{N}}$ decrescente é análogo.

Afirmação. Seja $\left\{x_{n}\right\}_{n \in \mathbb{N}} \subset X$ uma sequência convergente com $\lim x_{n}=x$. Então,

$$
\lim _{n \rightarrow+\infty} f\left(x_{n}\right)=f(x)
$$

Prova. Suponha por absurdo, que $\left\{f\left(x_{n}\right)\right\}_{n \in \mathbb{N}}$ não converge para $f(x)$, quando $n \rightarrow \infty$.

Então, existe subsequência $\left\{f\left(x_{n_{k}}\right)\right\}_{k \in \mathbb{N}}$, com $f(x)$ ponto não aderente. Isto é, existe uma vizinhança $V \subset Y$ que contém $f(x)$, tal que, para todo $k \in \mathbb{N}, f\left(x_{n_{k}}\right) \notin V$.

Agora, aplicando $f^{-1}$ temos a subsequência $\left\{x_{n_{k}}\right\}_{k \in \mathbb{N}}$ em $X$. E assim, podemos tomar uma subsubsequência monótona: $\left\{x_{n_{k_{j}}}\right\}_{j \in \mathbb{N}}$. 
Assim, $\left\{x_{n_{k_{j}}}\right\}_{j \in \mathbb{N}} \subset X$ é sequência monótona com $\lim _{j \rightarrow \infty} x_{n_{k_{j}}}=x$. Pela afirmação anterior,

$$
\lim _{j \rightarrow \infty} f\left(x_{n_{k_{j}}}\right)=f(x) .
$$

Absurdo. Pois, $f(x)$ é um ponto não aderente de $\left\{f\left(x_{n_{k}}\right)\right\}_{k \in \mathbb{N}}$.

Com a segunda afirmação, fica claro que $f: X \rightarrow Y$ é contínua. E de mesma forma, $f^{-1}: Y \rightarrow X$ é bijetiva e crescente, satisfazendo todas as hipóteses do enunciado. Portanto, temos de forma análoga $f^{-1}: Y \rightarrow X$ é contínua. Portanto, $f: X \rightarrow Y$ é homeomorfismo.

Definição 2.1.14 Dados $X, Y \subset \mathbb{R}$ subconjuntos, dizemos que $X$ é denso em $Y$ se para todo $y \in Y$, existe uma sequência $\left\{x_{n}\right\}_{n \in \mathbb{N}}$ em $X$, com

$$
\lim _{n \rightarrow+\infty} x_{n}=y
$$

Lema 2.1.15 Seja $C \subset \mathbb{R}$ subconjunto qualquer. Então, existe $D \subset C$ enumerável e denso em $C$, pela topologia induzida.

Prova. Defina:

$$
B(x, r):=\{y \in \mathbb{R} \mid d(x, y)<r\}
$$

onde, $d: \mathbb{R} \times \mathbb{R} \rightarrow \mathbb{R}$ é a distância euclidiana em $\mathbb{R}$.

Seja:

$$
\mathcal{B}:=\{B(x, r) \mid x \in \mathbb{Q}, r \in \mathbb{Q}\} .
$$

Note que $\mathcal{B}$ é base enumerável de $\mathbb{R}$. E por fim, defina:

$$
\mathcal{B}_{C}:=\{B \cap C \mid B \in \mathcal{B} \text { e } B \cap C \neq \emptyset\}
$$

E note que $\mathcal{B}_{C}$ é uma base enumerável de $C$, para a topologia induzida. Agora basta escolher, para cada $B \in \mathcal{B}_{C}$, um representante $x_{B} \in B$. E seja:

$$
D:=\left\{x_{B} \mid B \in \mathcal{B}_{C}\right\}
$$

É fácil notar que $D \subset C$ é subconjunto enumerável e denso.

Observação 2.1.16 De forma análoga, podemos demonstrar que: qualquer subconjunto de um espaço métrico separável, é separável na métrica induzida.

Lema 2.1.17 Sejam $X \subset \mathbb{R}$ subconjunto fechado bi-ilimitado, $D_{X} \subset X$ e $D \subset \mathbb{R}$ subconjuntos densos. Se existe $h_{0}: D_{X} \rightarrow D$ crescente e sobrejetiva (respectivamente bijetiva) então, existe uma única extensão de $h_{0} . h: X \rightarrow \mathbb{R}$ 
crescente e sobrejetiva, com $\left.h\right|_{D_{X}}=h_{0}$ (e $h$ bijeção em $X \backslash \overline{X^{c}}$, no caso de $h_{0}$ bijetiva).

Além disso, para cada componente conexa $(a, b)$ do complementar de $X$, temos: $h(a)=h(b)$.

Prova. Defina $h_{-}, h_{+}: X \rightarrow \mathbb{R}$, como:

$$
\begin{aligned}
& h_{-}(x)=\sup \left\{h_{0}(y) \mid y \in D_{X} \text { e } y \leq x\right\} \\
& h_{+}(x)=\inf \left\{h_{0}(y) \mid y \in D_{X} \text { e } y \geq x\right\} .
\end{aligned}
$$

Note que, $h_{-}, h_{+}$são funções crescentes.

Afirmação. $\quad h_{-}(x)=h_{+}(x)$, para todo $x \in X$.

Prova. Segue direto da definição que $h_{-}(x) \leq h_{+}(x)$. Logo, basta mostrar que não existe $x \in X$, tal que: $h_{-}(x)<h_{+}(x)$.

Note que, para todo $x \in D_{X}$, como $h_{0}$ é crescente, temos:

$$
\begin{aligned}
& h_{-}(x)=\sup \left\{h_{0}(y) \mid y \in D_{X} \text { e } y \leq x\right\}=\left\{h_{0}(y) \mid y=x\right\}=h_{0}(x), \\
& h_{+}(x)=\inf \left\{h_{0}(y) \mid y \in D_{X} \text { e } y \geq x\right\}=\left\{h_{0}(y) \mid y=x\right\}=h_{0}(x) .
\end{aligned}
$$

Portanto, $h_{-}(x)=h_{+}(x)$, para todo $x \in D_{X}$.

Suponha por absurdo, que existe $x \in X$ com $h_{-}(x)<h_{+}(x)$ (portanto, $\left.x \notin D_{X}\right)$. Note que $\left(h_{-}(x), h_{+}(x)\right) \subset \mathbb{R}$ é um subconjunto aberto não-vazio, e portanto:

$$
\left(h_{-}(x), h_{+}(x)\right) \cap D \neq \emptyset .
$$

Escolha $y \in\left(h_{-}(x), h_{+}(x)\right) \cap D$. Como $h_{0}$ é sobrejetiva, temos: $h_{0}^{-1}(y) \neq \emptyset$. Porém,

$$
\begin{aligned}
& y>h_{-}(x) \Rightarrow h_{0}^{-1}(y) \cap(-\infty, x)=\emptyset, \\
& y<h_{+}(x) \Rightarrow h_{0}^{-1}(y) \cap(x,+\infty)=\emptyset .
\end{aligned}
$$

O que implica em $h_{0}^{-1}(y)=\{x\}$. Absurdo, pois $h_{0}^{-1}(y) \subset D_{X}$ e $x \notin D_{X}$.

Afirmação. Se existe extensão $h: X \rightarrow \mathbb{R}$ crescente, com $\left.h\right|_{D_{X}}=h_{0}$, então ela é única, e igual à $h_{+}$.

Prova. Basta notar que, se $h$ é extensão crescente, então:

$h(x)=\sup \{h(y) \mid y \leq x\} \geq \sup \left\{h_{0}(y) \mid y \in D_{X}\right.$ e $\left.y \leq x\right\}=\sup \left\{h(y) \mid y \in D_{X}\right.$ e $\left.y \leq x\right\}=h_{-}(x)$

$h(x)=\inf \{h(y) \mid y \geq x\} \leq \inf \left\{h_{0}(y) \mid y \in D_{X}\right.$ e $\left.y \geq x\right\}=\inf \left\{h(y) \mid y \in D_{X}\right.$ e $\left.y \geq x\right\}=h_{+}(x)$. 
Portanto, temos:

$$
h_{-}(x) \leq h(x) \leq h_{+}(x) \text {, para todo } x \in X \text {. }
$$

Mas, como vimos na última afirmação, $h_{-}(x)=h_{+}(x)$, e portanto:

$$
h(x)=h_{-}(x)=h_{+}(x), \text { para todo } x \in X .
$$

Assim, a extensão é única.

Afirmação. $h_{+}: X \rightarrow \mathbb{R}$ é sobrejetiva.

Prova. Tome $y \in \mathbb{R}$. Caso $y \in D$, sabemos que existe $x \in D_{X}$, com $h_{0}(x)=y$. Mas sabemos que $h_{+}$é extensão, portanto: $h_{+}(x)=h_{0}(x)=y$.

Caso $y \notin D$, existe sequência monótona decrescente em $D$, que converge para $y$ :

$$
\left(y_{n}\right)_{n \in \mathbb{N}} \text {, satisfazendo: } y_{n} \geq y_{n+1} \text {, e } y_{n} \rightarrow y \text {. }
$$

Sabemos que cada $h_{+}^{-1}\left(y_{n}\right) \neq \emptyset$, então escolhemos $x_{n} \in h_{+}^{-1}\left(y_{n}\right)$. Note que $\left(x_{n}\right)_{n \in \mathbb{N}}$ é sequência monótona decrescente e limitada inferiormente. Portanto, $\left(x_{n}\right)_{n \in \mathbb{N}}$ é convergente.

Seja,

$$
x:=\lim _{n \rightarrow+\infty} x_{n}
$$

Vamos calcular $h_{+}(x)$,

$$
h_{+}(x)=\inf \left\{h_{0}(y) \mid y \in D_{X} \text { e } y \geq x\right\}=\lim _{n \in \mathbb{N}} h_{0}\left(x_{n}\right)=\lim _{n \in \mathbb{N}} y_{n}=y .
$$

Portanto, $h_{+}$é sobrejetiva.

Afirmação. Se $h_{0}$ é bijetiva, então $h_{+}$também é bijetiva em $X \backslash \overline{X^{c}}$.

Prova. Seja $x_{1}<x_{2}$. Como $h_{0}$ é bijetiva, temos:

$$
\begin{gathered}
h_{+}\left(x_{1}\right)=h_{+}\left(x_{2}\right) \Leftrightarrow \inf \left\{h_{0}(y) \mid y \in D_{X} \text { e } y \geq x_{1}\right\}=\inf \left\{h_{0}(y) \mid y \in D_{X} \text { e } y \geq x_{2}\right\} \\
\Leftrightarrow\left\{h_{0}(y) \mid y \in D_{X} \text { e } y \geq x_{1}\right\}=\left\{h_{0}(y) \mid y \in D_{X} \text { e } y \geq x_{2}\right\} \\
\Leftrightarrow\left\{y \mid y \in D_{X} \text { e } y \geq x_{1}\right\}=\left\{y \mid y \in D_{X} \text { e } y \geq x_{2}\right\} \\
\Rightarrow\left(x_{1}, x_{2}\right) \cap D_{X}=\emptyset \Rightarrow x_{1}, x_{2} \in \overline{X^{c}} .
\end{gathered}
$$

Portanto, $h_{+}$é bijetiva em $\mathbb{R} \backslash \overline{X^{c}}=X \backslash \overline{X^{c}}$.

Basta escolher $h:=h_{+}$, pois assim temos uma extensão crescente e sobrejetiva de $h_{0}$. E note que, dado uma componente $(a, b)$ do complementar de $X$, se $h(a)<h(b)$, então $(h(a), h(b))$ é um intervalo aberto não contido na 
imagem de $h$, o que é um absurdo.

Portanto, $h: X \rightarrow \mathbb{R}$ satisfaz todas as conclusões do lema.

Observação 2.1.18 Nas mesmas hipóteses, $h$ também pode ser estendida, de forma única, a uma função crescente $\tilde{h}: \mathbb{R} \rightarrow \mathbb{R}$. Basta tomar, para cada componente conexa $(a, b)$ do complementar de $X$ :

$$
\tilde{h}(x)=h(a)=h(b), \text { para todo } x \in(a, b)
$$

Lema 2.1.19 Seja $F \subset \mathbb{R}$ fechado, ilimitado e sem pontos isolados. Então, existe $h: F \rightarrow \mathbb{R}$ crescente e sobrejetiva, com $h$ bijetiva em $F \backslash \overline{F^{c}}$.

Prova. Seja $F^{c}=\mathbb{R} \backslash F$ o conjunto complementar. Note que $F^{c} \subset \mathbb{R}$ é aberto, e portanto, pode ser representado pela união disjunta:

$$
F^{c}=\bigsqcup_{n \in \mathbb{N}}\left(a_{n}, b_{n}\right)
$$

Sejam,

$$
A:=\bigcup_{n \in \mathbb{N}}\left\{a_{n}\right\} \text { e } B:=\bigcup_{n \in \mathbb{N}}\left\{b_{n}\right\} .
$$

Note que $A$ e $B$ são disjuntos, caso contrário, existiria $x=a_{n}=b_{m}$ e note que:

$$
\left(a_{m}, x\right) \cup\left(x, b_{n}\right) \subset F^{c} \Rightarrow x \in F \text { é ponto isolado. }
$$

Defina $F_{0}:=F \backslash B$.

Afirmação. $F_{0} \subset F$ é subconjunto denso.

Prova. Basta mostrar que $F_{0}$ é denso em $B$. Tome $b_{n} \in B$ e $\epsilon>0$ quaisquer. Como $b_{n} \in F$, conjunto sem pontos isolados, portanto, a bola $B\left(b_{n}, \epsilon\right)$ intersecta o conjunto $F$ em outro ponto diferente de $b_{n}$. Portanto, existe $x \in F$ diferente de $b_{n}$ em $B\left(b_{n}, \epsilon\right)$.

Caso $x \in F_{0}$, temos:

$$
B\left(b_{n}, \epsilon\right) \cap F_{0} \neq \emptyset
$$

e não há mais nada a provar.

Caso $x \notin F_{0}$, implica em $x=b_{m} \in B$ diferente de $b_{n}$.

Agora, se $b_{m}<b_{n}$, note que como $\left(a_{m}, b_{m}\right)$ e $\left(a_{n}, b_{n}\right)$ são disjuntos, temos:

$$
b_{m}<a_{n}<b_{n} \Rightarrow a_{n} \in\left(b_{m}, b_{n}\right) \subset B\left(b_{n}, \epsilon\right)
$$

E como $a_{n} \in F_{0}$, temos: $B\left(b_{n}, \epsilon\right) \cap F_{0} \neq \emptyset$. 
Se $b_{m}>b_{n}$, como $\left(a_{m}, b_{m}\right)$ e $\left(a_{n}, b_{n}\right)$ são disjuntos, temos:

$$
b_{n}<a_{m}<b_{m} \Rightarrow a_{m} \in\left(b_{n}, b_{m}\right) \subset B\left(b_{n}, \epsilon\right)
$$

E como $a_{m} \in F_{0}$, temos: $B\left(b_{n}, \epsilon\right) \cap F_{0} \neq \emptyset$.

Tome $D \subset F_{0}$ subconjunto denso (e assim, denso em $F$ também) e enumerável. Portanto, $D=\left\{x_{0}, x_{1}, x_{2}, \ldots\right\}$. Defina agora:

$$
\alpha_{n}:=\min \left\{x_{0}, \ldots, x_{n-1}\right\} \text { e } \beta_{n}:=\max \left\{x_{0}, \ldots, x_{n-1}\right\}
$$

Seja $\mathcal{D} \subset \mathbb{R}$ o conjunto diádico,

$$
\mathcal{D}:=\left\{\frac{k}{2^{n}} \mid k \in \mathbb{Z}, n \in \mathbb{N}\right\}
$$

vamos construir de forma indutiva a seguinte função $h_{D}: D \rightarrow \mathcal{D}$ :

$-h_{D}\left(x_{0}\right)=0$

- se $x_{n}<\alpha_{n}$, então $h_{D}\left(x_{n}\right)=h_{D}\left(\alpha_{n}\right)-1$,

- se $x_{n}>\beta_{n}$, então $h_{D}\left(x_{n}\right)=h_{D}\left(\beta_{n}\right)+1$,

- se $\alpha_{n}<x_{n}<\beta_{n}$, então $h_{D}\left(x_{n}\right)=\frac{h_{D}\left(x_{k_{1}}\right)+h_{D}\left(x_{k_{2}}\right)}{2}$.

onde $k_{1}:=\max _{i \in\{0, \ldots, n-1\}}\left\{x_{i}<x_{n}\right\}$ e $k_{2}:=\min _{i \in\{0, \ldots, n-1\}}\left\{x_{i}>x_{n}\right\}$.

Afirmação. $\quad h_{D}: D \rightarrow \mathcal{D}$ é crescente e sobrejetiva.

Prova. Note que $h_{D}$ é claramente crescente, pela construção. E como $D$ é denso em $F$, temos que $D$ é bi-ilimitado e sem pontos isolados. Além disso, como $D \subset F_{0}=F \backslash B$, temos que:

Para todos $x, y \in D \operatorname{com} x<y . \quad(x, y) \cap D \neq \emptyset$.

Tomamos então um elemento qualquer de $\mathcal{D}, \frac{k}{2^{n}}$. Vamos demonstrar sobrejetividade por indução sobre $n$ :

$-n=0$ :

Como $D$ é bi-ilimitado, para todo $n_{0} \in \mathbb{N}$, existem:

$$
n_{1}, n_{2}>n_{0}, \operatorname{com} x_{n_{1}}<\alpha_{n_{1}} \text { e } x_{n_{2}}>\beta_{n_{2}} .
$$

E portanto, para todo $n_{0} \in \mathbb{N}$, tanto $-n_{0}$ quanto $n_{0}$ pertencem à imagem de $h_{D}$. Assim, todos os números da forma $\frac{k}{2^{0}} \operatorname{com} k \in \mathbb{Z}$ pertencem à imagem. 
- Passo Indutivo:

Supomos que todos os números da forma $\frac{k}{2^{n}}$ com $k \in \mathbb{Z}$ pertencem à imagem de $h_{D}$. Note que números da forma $\frac{2 k}{2^{n+1}}=\frac{k}{2^{n}}$, portanto, naturalmente já pertencem à imagem de $h_{D}$. Basta mostrar que todos os números da forma $\frac{2 k+1}{2^{n+1}}$ também pertencem a imagem.

Para isso, sejam $x_{n_{k}}$ e $x_{n_{k}^{\prime}}$ as pré-imagens de $\frac{k}{2^{n}}$ e $\frac{k+1}{2^{n}}$, respectivamente. Pela propriedade descrita acima, temos $\left(x_{n_{k}}, x_{n_{k}^{\prime}}\right) \cap D \neq \emptyset$, então escolha $x_{m} \mathrm{o}$ primeiro número do processo de indução que está nesse intervalo. E portanto, segue que:

$$
h_{D}\left(x_{m}\right)=\frac{\frac{k}{2^{n}}+\frac{k+1}{2^{n}}}{2}=\frac{2 k+1}{2^{n+1}} .
$$

E assim, temos que todos da forma $\frac{k}{2^{n+1}}$, com $k \in \mathbb{Z}$ pertencem à imagem de $h_{D}$.

Concluindo assim, a prova da afirmação.

Pelo Lema 2.1.17, como $D \subset F$ e $\mathcal{D} \subset \mathbb{R}$ são subconjuntos densos, existe uma única extensão $h: F \rightarrow \mathbb{R}$ crescente e sobrejetiva, com $\left.h\right|_{D}=h_{D}$, e $h$ bijetiva em $F \backslash \overline{F^{c}}$. O que conclui a prova do lema.

Observação 2.1.20 Nas hipóteses do lema anterior, seja $(a, b) \subset F^{c}$ uma componente conexa do complementar de F. Então, $h(a)=h(b)$. Caso contrário, $(h(a), h(b))$ seria um aberto não-vazio, não contido na imagem de $h$, contradizendo a sobrejetividade de $h$.

Lema 2.1.21 Seja $f: \mathbb{R} \rightarrow \mathbb{R}$ crescente e sobrejetiva. Então, $f$ é contínua.

Prova.

Afirmação. Seja $x \in \mathbb{R}$, então a pré-imagem $f^{-1}(a)$ é um ponto ou um intervalo fechado.

Prova. Como $f$ é sobrejetiva, $f^{-1}(a) \neq \emptyset$, restando duas opções: $f^{-1}(a)$ igual à um único ponto, ou $f^{-1}(a)$ contém mais de um ponto.

Na primeira opção, não há nada à demonstrar. Na segunda opção, note que:

Se $x_{1}, x_{2} \in \mathbb{R}, \operatorname{com} f\left(x_{1}\right)=f\left(x_{2}\right) \Rightarrow f(y)=f\left(x_{1}\right), \quad \forall y \in\left(x_{1}, x_{2}\right)$.

Portanto, $f^{-1}(a)$ é necessariamente um intervalo.

Para concluir a prova, note que:

$$
\sup \{y \mid f(y)=a\} \in f^{-1}(a) \text { e } \inf \{y \mid f(y)=a\} \in f^{-1}(a) .
$$


Caso contrário, seja $\alpha=\sup \{y \mid f(y)=a\}$, teríamos $f(\alpha)>a$. E defina

$$
\alpha^{\prime}:=\frac{f(\alpha)+a}{2} .
$$

Agora,

$$
\begin{gathered}
\alpha^{\prime}<f(\alpha) \Rightarrow f^{-1}\left(\alpha^{\prime}\right) \cap[\alpha,+\infty)=\emptyset, \\
\alpha^{\prime}>a \Rightarrow f^{-1}\left(\alpha^{\prime}\right) \geq \sup \{y \mid f(y)=a\}=\alpha .
\end{gathered}
$$

As duas linhas juntas implicam em: $f^{-1}\left(\alpha^{\prime}\right)=\emptyset$. Absurdo, pois $f$ é sobrejetiva. A demonstração para o inf é análoga.

Agora, tome $(a, b) \subset \mathbb{R}$ subconjunto aberto. Vamos calcular a pré-imagem de $(a, b)$ por $f$. Sejam,

$$
\begin{gathered}
\alpha=\max \{x \mid f(x)=a\} \text { e } \beta=\min \{x \mid f(x)=b\} \\
\left.\left.f^{-1}(a, b)=\{x \mid a<f(x)<b)\right\}=\{x \mid \alpha<x<\beta)\right\}=(\alpha, \beta) .
\end{gathered}
$$

Concluindo, seja $A \subset \mathbb{R}$ um aberto qualquer. Podemos escrever: $A=$ $\bigcup_{i \in I}\left(a_{i}, b_{i}\right)$, com $\left\{a_{i}\right\}$ e $\left\{b_{i}\right\}$ sequências de números reais. Assim, para cada $i \in I$ definimos $\alpha_{i}$ e $\beta_{i}$ de forma análoga, e temos:

$$
f^{-1}(A)=f^{-1}\left(\bigcup_{i \in I}\left(a_{i}, b_{i}\right)\right)=\bigcup_{i \in I} f^{-1}\left(a_{i}, b_{i}\right)=\bigcup_{i \in I}\left(\alpha_{i}, \beta_{i}\right)
$$

Portanto, a pré-imagem de qualquer subconjunto aberto, é aberta. E assim, $f: \mathbb{R} \rightarrow \mathbb{R}$ é contínua.

Observação 2.1.22 Caso $f: \mathbb{R} \rightarrow \mathbb{R}$ crescente e bijetiva. Então, $f$ é homeomorfismo. Basta aplicar o Lema 2.1.21 para $f^{-1}$.

Corolário 2.1.23 Seja $F \subset \mathbb{R}$ fechado, ilimitado e sem pontos isolados. Então, existe $\tilde{h}: \mathbb{R} \rightarrow \mathbb{R}$ crescente, sobrejetiva e contínua, com $\tilde{h}$ constante em cada componente do complementar de F. Além disso, $\tilde{h}$ bijetiva em $F \backslash \overline{F^{c}}$.

Prova. Pelo Lema 2.1.17, existe $h: F \rightarrow \mathbb{R}$ crescente e sobrejetiva, com $h(a)=h(b)$ se $(a, b)$ for componente conexa do complementar. E $h$ bijetiva em $F \backslash \overline{F^{c}}$.

Seja $F^{c}=\bigsqcup_{n \in \mathbb{N}}\left(a_{n}, b_{n}\right)$. Defina a extensão $\tilde{h}: \mathbb{R} \rightarrow \mathbb{R}$, como:

$-\tilde{h}(x)=h(x)$, se $x \in F$,

- $\tilde{h}(x)=h\left(a_{n}\right)$, se $x \in\left(a_{n}, b_{n}\right)$.

Note que $\tilde{h}$ permanece sobrejetiva e crescente. Portanto, pelo Lema 2.1.21, $\tilde{h}$ é contínua. 
Lema 2.1.24 Dado $A \subset \mathbb{R}$ subconjunto enumerável ( $A$ pode ser finito). Então, existe $h: \mathbb{R} \rightarrow \mathbb{R}$ crescente, contínua e sobrejetiva, tal que:

- $h^{-1}(x)$ é um único ponto, se $x \notin A$.

- $h^{-1}(x)$ é um intervalo compacto, se $x \in A$.

Prova. Seja $A=\left\{a_{1}, a_{2}, a_{3}, \ldots\right\}$ o conjunto enumerável. Tome $\left\{c_{n}\right\}_{n \in \mathbb{N}} \subset \mathbb{R}$ uma sequência, com:

$$
\sum_{n=0}^{\infty} c_{n}<+\infty \quad \text { e } \quad c_{n}>0, \text { para todo } n \in \mathbb{N}
$$

Sejam,

$$
\begin{aligned}
& I^{+}(x):=\left\{n \in \mathbb{N} \mid a_{n} \in[0, x)\right\}, \\
& I^{-}(x):=\left\{n \in \mathbb{N} \mid a_{n} \in[x, 0)\right\} .
\end{aligned}
$$

Defina a seguinte função $b: \mathbb{R} \rightarrow \mathbb{R}$ :

$$
b(x)=\left\{\begin{array}{c}
-\sum_{n \in I^{-}(x)} c_{n} \text { se } x<0, \\
\sum_{n \in I^{+}(x)} c_{n} \text { se } x \geq 0 .
\end{array}\right.
$$

Observe que $b$ é crescente e limitada.

Agora, vamos construir $h: \mathbb{R} \rightarrow \mathbb{R}$ da seguinte forma:

$$
h^{-1}(x)=\left\{\begin{aligned}
x+b(x) & \text { se } x \notin A \\
{\left[x+b(x), x+b(x)+c_{n}\right] } & \text { se } x=a_{n}
\end{aligned}\right.
$$

Afirmação. $h: \mathbb{R} \rightarrow \mathbb{R}$ é função bem definida.

Prova. Para que $h$ seja bem definida é necessário, e suficiente, que suas préimagens $h^{-1}(x)$ sejam disjuntas, para todo $x \in \mathbb{R}$ e que a união delas cubra todo o domínio $\mathbb{R}$. Note que $\bigcup_{x \in \mathbb{R}}\{x+b(x)\}$ cobre todo o domínio, exceto pelos saltos dados pela função $b(x)$ nos valores $a_{n}$. Portanto,

$$
\begin{gathered}
\mathbb{R}=\bigcup_{x \in \mathbb{R}}\{x+b(x)\} \cup \bigcup_{n \in \mathbb{N}}\left(a_{n}+b\left(a_{n}\right), a_{n}+b\left(a_{n}\right)+c_{n}\right] \\
=\bigcup_{x \notin A}\{x+b(x)\} \cup \bigcup_{x \in A}\{x+b(x)\} \cup \bigcup_{n \in \mathbb{N}}\left(a_{n}+b\left(a_{n}\right), a_{n}+b\left(a_{n}\right)+c_{n}\right] \\
=\bigcup_{x \notin A}\{x+b(x)\} \cup \bigcup_{n \in \mathbb{N}}\left[a_{n}+b\left(a_{n}\right), a_{n}+b\left(a_{n}\right)+c_{n}\right]=\bigcup_{x \in \mathbb{R}} h^{-1}(x) .
\end{gathered}
$$

E assim, a união das pré-imagens cobre o domínio $\mathbb{R}$. 
Por fim, basta perceber que se $x<y$, então $b(x) \leq b(y)$, assim:

$$
x+b(x)<y+b(x) \leq y+b(y) .
$$

Portanto, $h^{-1}(x) \cap h^{-1}(y)=\emptyset$, para todo $x \notin A$ e todo $y \in \mathbb{R}$.

Caso $x=a_{n}$, note que $b(y) \geq b\left(a_{n}\right)+c_{n}$, pois $y>a_{n}$. Assim:

$$
a_{n}+b\left(a_{n}\right)+c_{n} \leq a_{n}+b(y)<y+b(y) .
$$

Portanto, $h^{-1}\left(a_{n}\right) \cap h^{-1}(y)=\emptyset$, para todo $a_{n} \in A$ e todo $y \in \mathbb{R}$.

Concluindo que as pré-imagens são todas disjuntas dois-à-dois. E portanto, $h: \mathbb{R} \rightarrow \mathbb{R}$ é função bem definida.

Agora que $h$ é uma função bem definida, é fácil notar que ela satisfaz os dois itens. Além disso, $h$ é sobrejetiva por definição, e é crescente, pelo Lema 2.1.6, portanto $h: \mathbb{R} \rightarrow \mathbb{R}$ é contínua, pelo Lema 2.1.21.

\section{2}

\section{Ação de grupos}

O sistema dinâmico mais simples é, sem dúvidas, um sistema discreto definido por uma bijeção $f$. Uma forma muito natural de estuda-lo é definir uma aplicação da forma: $(k, x) \mapsto f^{k}(x)$. Ou se temos um sistema contínuo, dado um fluxo $f_{t}$, definimos a aplicação: $(t, x) \mapsto f_{t}(x)$. Ou seja, temos aplicações da forma:

$$
\varphi: \mathbb{Z} \times X \rightarrow X \quad \text { e } \quad \psi: \mathbb{R} \times X \rightarrow X
$$

Um passo natural é trocar $\mathbb{Z}$ ou $\mathbb{R}$ por um grupo $G$ qualquer. Verificando algumas propriedades (veja a Definição 2.2.8), essa aplicação $\phi: G \times X \rightarrow X$ será chamada de ação de grupo, e o que vamos estudar, são hipóteses dinâmicas sobre a aplicação $\phi$ que resultam em conclusões algébricas sobre o grupo $G$.

Mas antes disso, temos que começar com as primeiras definições:

Definição 2.2.1 Um conjunto $G$ munido de uma operação binária $\star$ : $G \times$ $G \rightarrow G$ satisfazendo, para todo $a, b, c \in G$ :

$-(a \star b) \star c=a \star(b \star c)$ (Associatividade),

- existe um elemento $I d \in G$, tal que: $I d \star a=a \star I d=a$ (Elemento Neutro),

- para cada $a \in G$, existe $a^{\prime} \in G$ com: $a \star a^{\prime}=a^{\prime} \star a=I d$ (Elemento Inverso), 
então, $(G, \star)$ é chamado de grupo.

Caso $G$ e também satisfaça, para todo $a, b \in G$ :

$-a \star b=b \star a$ (Comutatividade),

então, $(G, \star)$ é chamado de grupo abeliano.

Denotaremos como $\mathrm{Homeo}_{+}(\mathbb{R})$, o conjunto dos homeomorfismos crescentes de $\mathbb{R}$.

Exemplo 2.2.2 $\left(\mathrm{HomeO}_{+}(\mathbb{R})\right.$, o) é um grupo (não-abeliano), onde o é a composição de funções.

Definição 2.2.3 Um conjunto $G$ munido de uma operação binária * e uma relação binária $\prec$ é chamado de grupo totalmente ordenado, se $(G, \star)$ é um grupo e $(G, \prec)$ é um conjunto totalmente ordenado. Nesse caso usamos a notação $(G, \star, \prec)$.

Definição 2.2.4 Um grupo totalmente ordenado $(G, \star, \prec)$ é dito invariante por multiplicação à esquerda se dados $a, b \in G$ então para todo $c \in G$, temos

$$
a \prec b \Rightarrow c \star a \prec c \star b .
$$

O grupo é dito invariante por multiplicação à direita se

$$
a \prec b \Rightarrow a \star c \prec b \star c .
$$

Definição 2.2.5 Um grupo totalmente ordenado $(G, \star, \prec)$ é arquimediano se para todo $a, b \in G$ com $a \succ I d$ e $b \succ I d$ se verifica, que

$$
\text { existe } n \in \mathbb{N} \text {, com } a \prec b^{n}=b \star b \star \ldots \star b \text {. }
$$

Exemplo 2.2.6 $(\mathbb{R},+,<)$ é um grupo totalmente ordenado arquimediano.

Denotaremos por $\mathbb{Z} . s$ o conjunto de todos os múltiplos inteiros de $s$, ou seja, $\mathbb{Z} . s:=\{k . s \mid k \in \mathbb{Z}\}$.

Lema 2.2.7 Seja $S$ um subgrupo de $(\mathbb{R},+)$. Então:

- ou $S$ é gerado por um único elemento (ou seja, $S=\mathbb{Z} . s$ ). 
- ou $S$ é denso sobre $\mathbb{R}$.

Prova. Defina $s_{0}:=\inf \{s \in S \mid s>0\}$.

Afirmação. $\mathbb{Z} . s_{0} \in S$. Além disso, se $s_{0} \neq 0$ então $\mathbb{Z} . s_{0}=S$.

Prova. Se $s_{0}=0$, então $\mathbb{Z} . s_{0}=\{0\}$ que pertence ao $S$ por definição de subgrupo. Agora, podemos supor que $s_{0}>0$. Suponha por absurdo, que $s_{0} \notin S$. Como $s_{0}$ é o ínfimo, temos que para todo $\epsilon>0$ se verifica que

existe $s \in S$ tal que $s_{0}<s<s_{0}+\epsilon$.

Como $s_{0}>0$ podemos escolher $\epsilon<s_{0}$. Implicando em

existe $s_{1} \in S$, tal que $s_{0}<s_{1}<2 s_{0}$.

Agora, como $s_{1}>s_{0}$, podemos escolher um segundo $\epsilon<s_{1}-s_{0}$, e assim

existe $s_{2} \in S$, tal que $s_{0}<s_{2}<s_{1}$.

Concluindo assim, que $s_{1}, s_{2} \in\left(s_{0}, 2 s_{0}\right)$, portanto

$$
0<s_{1}-s_{2}<s_{0}
$$

Como $s_{1}-s_{2} \in S$, temos uma contradição com a definição de $s_{0}$. Provamos assim a primeira parte da afirmação.

Para a segunda parte, suponha por absurdo que existe $s^{\prime} \in S$ tal que $s^{\prime} \notin \mathbb{Z} . s_{0}$. Então, existe $n \in \mathbb{Z}$, com $s_{0} . n<s^{\prime}<s_{0} .(n+1)$. E assim, $0<s^{\prime}-s_{0} . n<s_{0}$. Como $s^{\prime}-s_{0} . n \in S$, temos uma contradição com a definição de $s_{0}$.

Agora existem dois casos: se $s_{0}>0$ temos $S=\mathbb{Z} . s_{0}$ e assim, estamos no primeiro caso do Lema. Caso $s_{0}=0, S$ se acumula no ponto 0 . E como $S$ tem estrutura de grupo com a operação + , ele se acumula em todos os pontos de $\mathbb{R}$. Portanto, $S$ é denso em $\mathbb{R}$, provando o Lema.

Definição 2.2.8 Dados um grupo $(G, \star)$ e um conjunto $X$ uma ação de grupos $\phi$ de $G$ em $X$ é uma aplicação

$$
\phi: G \times X \rightarrow X
$$

satisfazendo, as seguintes condições para todo $x \in X$ e todo $g_{1}, g_{2} \in G$ :

- $\phi(I d, x)=x$ (Identidade),

- $\phi\left(g_{1} \star g_{2}, x\right)=\phi\left(g_{1}, \phi\left(g_{2}, x\right)\right)$ (Compatibilidade). 
A aplicação $\phi$ é dita fiel se para todo $g \in G$ se verifica:

- se $g \neq I d$, então existe $x \in \mathbb{R} \operatorname{com} \phi(g, x) \neq x$.

Observação 2.2.9 Podemos definir para cada $g \in G$ a aplicação $\phi(g): X \rightarrow$ $X$, onde $\phi(g)(x)=\phi(g, x)$. Note que para cada $g$, $\phi(g)$ é uma bijeção com $\phi\left(g^{-1}\right)$ sendo sua inversa. Podemos então, definir a ação $\phi$ como:

$$
\phi: G \rightarrow \operatorname{Bij}(X)
$$

onde $\operatorname{Bij}(X)$ é o conjunto de bijeções de $X$. Vamos usar essa notação quando conveniente.

Observação 2.2.10 Dado um espaço topológico $X$, dizemos $\phi: G \times X \rightarrow X$ é uma ação de grupos contínua se para cada $g \in G$ a aplicação $x \mapsto \phi(g)(x)$ é um homeomorfismo. Em outras palavras, $\phi: G \rightarrow \operatorname{Homeo}(X)$ ou também, $\phi(G) \subset \operatorname{Homeo}(X)$ é um subgrupo.

Exemplo 2.2.11 Dado um subgrupo $G \subset$ Homeo $_{+}(\mathbb{R})$ existe a ação natural $\phi$ sobre $\mathbb{R}$ definida por:

$$
\phi(g, x) \mapsto g(x)
$$

Nesse caso, omitiremos a aplicação $\phi$.

Definição 2.2.12 Sejam $\phi: G \times X \rightarrow X$ uma ação de grupos e $x \in X$. A órbita de $x$ é o conjunto $G . x \subset X$ definido por:

$$
G . x:=\{\phi(g, x) \in X \mid g \in G\} .
$$

Lema 2.2.13 Sejam $G \subset \mathrm{Homeo}_{+}(\mathbb{R})$ e $x \in \mathbb{R}$. Então:

$$
\text { G.x é finita } \Longleftrightarrow G . x=\{x\} \text {. }
$$

Prova. A implicação $\Leftarrow$ é óbvia. Para a implicação $\Rightarrow$ suponha por absurdo que existe uma órbita com mais de um elemento:

$$
G . x=\left\{x_{0}, x_{1}, \ldots, x_{m}\right\} \text { com } x_{0}<x_{1}<\cdots<x_{m} .
$$

Note que, existe $g \in G$ tal que: $g\left(x_{0}\right)=x_{m}$. Porém,

$$
g\left(x_{m}\right) \in G \cdot x \Rightarrow g\left(x_{m}\right) \in\left\{x_{0}, x_{1}, \ldots, x_{m}\right\} \Rightarrow g\left(x_{m}\right) \leq x_{m} .
$$


Portanto, temos: $g\left(x_{0}\right)=x_{m} \geq g\left(x_{m}\right)$. Isto é um absurdo, pois $x_{0}<x_{m}$ e $g: \mathbb{R} \rightarrow \mathbb{R}$ é estritamente crescente. Assim, G.x é um único ponto.

Lema 2.2.14 Considere $g: \mathbb{R} \rightarrow \mathbb{R}$ um homeomorfismo crescente e um intervalo $(a, b)$, onde $a \in \mathbb{R} \cup\{-\infty\}, b \in \mathbb{R} \cup\{+\infty\}$.

Suponha que $g(x)>x$ para todo $x \in(a, b), g(a)=a($ se $a \neq-\infty) e$ $g(b)=b($ se $b \neq+\infty)$.

Então para todo $x \in(a, b)$ se verifica

$$
\lim _{n \rightarrow+\infty} g^{n}(x)=b e \lim _{n \rightarrow+\infty} g^{-n}(x)=a .
$$

Prova. Note que:

$$
x<g(x)<g(g(x))<g(g(g(x)))<\ldots<g(b)=b,
$$

onde escrevemos $g(+\infty)=+\infty$.

Portanto, para todo $x \in \mathbb{R},\left\{g^{n}(x)\right\}_{n \in \mathbb{N}}$ é uma sequência crescente limitada por $b$. Então ela é "convergente"(o limite pode ser $+\infty)$. Defina: $c=\lim _{n \rightarrow+\infty} g^{n}(x)$. Por continuidade temos:

$$
g(c)=g\left(\lim _{n \rightarrow+\infty} g^{n}(x)\right)=\lim _{n \rightarrow+\infty} g\left(g^{n}(x)\right)=\lim _{n \rightarrow+\infty} g^{n+1}(x)=c .
$$

Assim, como $g(x)>x$ para todo $x \in(a, b)$, segue que $c=b$.

O outro caso é análogo.

Definição 2.2.15 Sejam $X$ um conjunto e $f: X \rightarrow X$ uma aplicação. Definimos o subconjunto dos pontos fixos $\operatorname{Fix}(f) \subset X$ como:

$$
\operatorname{Fix}(f):=\{x \in X \mid f(x)=x\}
$$

Definição 2.2.16 Sejam $g: X \rightarrow X$ e $f: Y \rightarrow Y$ dois homeomorfismos crescentes. Dizemos que $g$ e $f$ são conjugados se existe $h: X \rightarrow Y$ homeomorfismo crescente tal que para todo $x \in X$, se verifica

$$
f \circ h(x)=h \circ g(x)
$$

Ou seja, o seguinte diagrama comuta: 


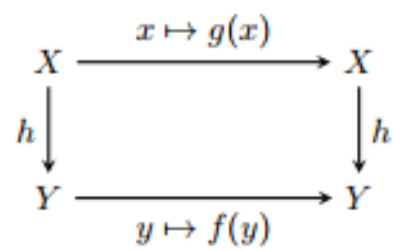

Observação 2.2.17 A conjugação é uma relação de equivalência. Com efeito:

- g é conjugado a g, pela identidade Id.

- Se g é conjugado a $f$ por $h$, então $f$ é conjugado a $g$ por $h^{-1}$.

- Se g é conjugado a $f$ por $h_{1}$, e $f$ é conjugado a $k$ por $h_{2}$, então $g$ é conjugado a $k$ por $h_{1} \circ h_{2}$.

Lema 2.2.18 Sejam $g: X \rightarrow X$ e $f: Y \rightarrow Y$ dois homeomorfismos conjugados por $h: X \rightarrow Y$. Então,

$$
\operatorname{Fix}(f)=h(\operatorname{Fix}(g))
$$

Em particular, \#Fix $(f)=\# \operatorname{Fix}(g)$.

Prova. Temos pela conjugação que, para todo $y \in Y$ se verifica:

$$
f(y)=h \circ g \circ h^{-1}(y)
$$

Tome então, $x_{0} \in \operatorname{Fix}(g)$. Assim:

$$
f\left(h\left(x_{0}\right)\right)=h \circ g \circ h^{-1}\left(h\left(x_{0}\right)\right)=h \circ g\left(x_{0}\right)=h\left(x_{0}\right) .
$$

Portanto, $h(\operatorname{Fix}(g)) \subset \operatorname{Fix}(f)$. Considerando agora a conjugação $g=h^{-1} \circ f \circ h$ e "trocando os papeis" de $f$ e $g$, obtemos: $h^{-1}(\operatorname{Fix}(f)) \subset \operatorname{Fix}(g)$. Assim, $\operatorname{Fix}(f) \subset h(\operatorname{Fix}(g))$ e $\operatorname{Fix}(f)=h(\operatorname{Fix}(g))$. Por fim, como $h: X \rightarrow Y$ é homeomorfismo, fica claro que: \# $\operatorname{Fix}(f)=\# \operatorname{Fix}(g)$.

Lema 2.2.19 Se g é homeomorfismo crescente tal que $g(x)>x$ para todo $x \in \mathbb{R}$, então g é conjugado à translação $x \mapsto x+1$.

Prova. Considere o intervalo $[0, g(0)]$ e tome a aplicação linear

$$
L:[0, g(0)] \rightarrow[0,1], \quad L(x)=\frac{1}{g(0)} x
$$


Agora, defina $h_{n}:\left[g^{n}(0), g^{n+1}(0)\right] \rightarrow[n, n+1]$,

$$
h_{n}(x)=L\left(g^{-n}(x)\right)+n .
$$

Note que $h_{n}$ é homeomorfismo crescente.

Observando que, pelo Lema 2.2.14 se verifica que $\mathbb{R}=\bigcup_{n \in \mathbb{Z}}\left[g^{n}(0), g^{n+1}(0)\right]$ podemos definir a aplicação $h: \mathbb{R} \rightarrow \mathbb{R}$

$$
h(x)=h_{n}(x) \text { se } x \in\left[g^{n}(0), g^{n+1}(0)\right] .
$$

Note que $h$ é homeomorfismo crescente em cada intervalo da forma $\left[g^{n}(0), g^{n+1}(0)\right]$, e como os intervalos se encaixam de forma ordenada, segue que $h$ é homeomorfismo crescente em $\mathbb{R}$. Por fim, note que dado $x \in\left[g^{n}(0), g^{n+1}(0)\right]$ se verifica

$$
\begin{gathered}
h \circ g(x)=h_{n+1}(g(x))=L\left(g^{-n-1}(g(x))+n+1\right. \\
=L\left(g^{-n}(x)\right)+n+1=h_{n}(x)+1=h(x)+1 .
\end{gathered}
$$

Portanto, segue que $h$ conjuga $g$ com a translação $x \mapsto x+1$.

Corolário 2.2.20 Se g é homeomorfismo crescente tal que $g(x)>x$ para todo $x \in \mathbb{R}$, então g é conjugado a qualquer translação positiva $x \mapsto x+t$ com $t>0$.

Prova. Note que a translação $x \mapsto x+t$ com $t>0$, também satisfaz o enunciado do Lema 2.2.19, e portanto é conjugada a $x \mapsto x+1$, por um homeomorfismo crescente $h^{\prime}$. E como temos que $g$ é conjugada a $x \mapsto x+1$ por $h$, basta tomar $h^{\prime-1} \circ h$ que temos uma conjugação entre $g$ e $x \mapsto x+t$.

Definição 2.2.21 Sejam $g: X \rightarrow X$ e $f: Y \rightarrow Y$ dois homeomorfismos crescentes. Dizemos que g é semi-conjugada a $f$, se existe $h: X \rightarrow Y$ contínua, sobrejetiva e crescente tal que para todo $x \in X$,

$$
f \circ h(x)=h \circ g(x)
$$

Ou seja, o diagrama abaixo comuta:

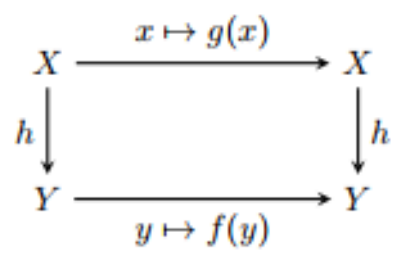


Observação 2.2.22 Diferentemente de conjugação, semi-conjugação não é uma relação de equivalência. Com efeito, podemos escolher $X=Y=\mathbb{R}$, $f(x)=x$ para todo $x \in \mathbb{R} e$

$$
g(x)=\left\{\begin{aligned}
x & \text { se } x<0 \\
x^{2} & \text { se } 0 \leq x \leq 1 \\
x & \text { se } x>1
\end{aligned}\right.
$$

Tomando $h: \mathbb{R} \rightarrow \mathbb{R}$ da forma:

$$
h(x)=\left\{\begin{aligned}
x & \text { se } x<0 \\
0 & \text { se } 0 \leq x \leq 1 \\
x-1 & \text { se } x>1
\end{aligned}\right.
$$

Note que $h$ é aplicação contínua, crescente, sobrejetiva e verifica para todo $x \in \mathbb{R}$, que $h \circ g(x)=f \circ h(x)$. Portanto, $g$ é semi-conjugada a $f$.

Porém, $f$ não é semi-conjugada a $g$. Pois, tomando $h^{\prime}: \mathbb{R} \rightarrow \mathbb{R}$ sobrejetiva e escolhendo $y \in \mathbb{R}$ tal que $h^{\prime}(y)=\frac{1}{2}$, temos:

$$
h^{\prime} \circ f(y)=h^{\prime}(y)=\frac{1}{2} \neq \frac{1}{4}=g\left(\frac{1}{2}\right)=g \circ h^{\prime}(y) .
$$

Ou seja, não existe aplicação $h^{\prime}$ sobrejetiva que satisfaça $h^{\prime} \circ f(y)=g \circ h^{\prime}(y)$.

Lema 2.2.23 Sejam $X, Y \subset \mathbb{R}$ e $g: X \rightarrow X, f: Y \rightarrow Y$ dois homeomorfismos semi-conjugados por $h: X \rightarrow Y$ contínua, sobrejetiva e crescente. Então,

$$
\operatorname{Fix}(g) \subset h^{-1}(\operatorname{Fix}(f))
$$

Além disso, $\operatorname{Fix}(g)=\emptyset \Leftrightarrow \operatorname{Fix}(f)=\emptyset$.

Prova. Pela semi-conjugação, temos que para todo $x \in X$, se verifica:

$$
f \circ h(x)=h \circ g(x)
$$

Para provar a inclusão tome qualquer $x_{0} \in \operatorname{Fix}(g)$ e observe que

$$
f \circ h\left(x_{0}\right)=h \circ g\left(x_{0}\right)=h\left(x_{0}\right)
$$

Portanto, $h\left(x_{0}\right) \in \operatorname{Fix}(f)$. Assim temos $\operatorname{Fix}(g) \subset h^{-1}(\operatorname{Fix}(f))$.

Como consequência direta, temos

$$
\operatorname{Fix}(f)=\emptyset \Rightarrow \operatorname{Fix}(g)=\emptyset \text {. }
$$


Para provar a recíproca vamos usar a contra-positiva, suponha portanto $\operatorname{Fix}(f) \neq \emptyset$. Considere $y_{0} \in \operatorname{Fix}(f)$. Como $h: X \rightarrow Y$ é contínua, crescente e sobrejetiva, $h^{-1}\left(y_{0}\right)$ é igual à um ponto ou um intervalo fechado limitado superiormente e inferiormente, em particular possui máximo. Tome então $x \in h^{-1}\left(y_{0}\right)$ qualquer e observe que

$$
h \circ g(x)=f \circ h(x)=f\left(y_{0}\right)=y_{0} .
$$

Portanto, $g(x) \in h^{-1}\left(y_{0}\right)$. Ou seja, $g: X \rightarrow X$ deixa $h^{-1}\left(y_{0}\right)$ invariante. Mas como $g$ é crescente e contínua, ela fixa o ponto máximo. $\operatorname{Assim}, \operatorname{Fix}(g) \neq \emptyset$, o que conclui a contra-positiva.

Definição 2.2.24 Seja $(G, \star)$ um grupo, um elemento $g \in G$ é dito não-trivial se $g \neq I d$. Observe que, dada uma ação fiel $\phi$ de $G$ sobre um conjunto $X$, se para todo $x \in X$ temos que $\phi(g)(x)=x$ então $g$ é trivial.

Definição 2.2.25 Sejam X um conjunto, $G$ um grupo e $\phi$ uma ação de grupos de $G$ sobre $X$. Um ponto $x \in X$ é chamado de ponto fixo, se existe $g \in G$ nãotrivial, tal que $\phi(g, x)=x$.

Além disso, um ponto $x_{0} \in X$ é chamado de ponto fixo global, se para todo $g \in G, \phi\left(g, x_{0}\right)=x_{0}$.

Definição 2.2.26 Dizemos que a ação $\phi: G \rightarrow \operatorname{Bij}(X)$ age com no máximo $N$ pontos fixos se para todo $g \in G$ temos:

$$
\# \operatorname{Fix}(\phi(g))>N \Rightarrow g=I d
$$

Em particular, se $G$ age com no máximo 0 pontos fixos dizemos que $G$ é livre de pontos fixos.

Observação 2.2.27 Sejam $X$ um conjunto, $G$ um grupo e $\phi$ uma ação de grupos de $G$ sobre $X$. Se $\phi$ é uma ação com no máximo $N$ pontos fixos e $N<\# X$. Então, a ação é fiel.

Com efeito, se $\phi(g)(x)=x$ para todo $x \in X$, então

$$
\# \operatorname{Fix}(\phi(g))=\# X>N \Rightarrow g=I d
$$

Portanto, a ação é fiel.

Lema 2.2.28 Se $G \subset$ Homeo $_{+}(\mathbb{R})$ é subgrupo abeliano e age com no máximo $N$ pontos fixos, então todos os pontos fixos de elementos de $G$ são pontos fixos globais. 
Prova. Tome $g \in G$ não-trivial e considere seu conjunto de pontos fixos:

$$
\operatorname{Fix}(g)=\left\{x_{1}, x_{2}, \ldots, x_{k}\right\} \text { onde } k \leq N
$$

Agora, tome um elemento não-trivial $f \in G$ qualquer. Como $G$ é abeliano, temos para todo $x \in \mathbb{R}$, que

$$
g \circ f(x)=f \circ g(x)
$$

Fixe $x_{j} \in \operatorname{Fix}(g)$, então

$$
g \circ f\left(x_{j}\right)=f \circ g\left(x_{j}\right)=f\left(x_{j}\right)
$$

portanto, $f(\operatorname{Fix}(g)) \subset \operatorname{Fix}(g)$. E como $f: \mathbb{R} \rightarrow \mathbb{R}$ é um homeomorfismo crescente, $f$ define uma bijeção crescente em $\operatorname{Fix}(g)$,

$$
f:\left\{x_{1}, x_{2}, \ldots, x_{k}\right\} \rightarrow\left\{x_{1}, x_{2}, \ldots, x_{k}\right\}
$$

Porém, a identidade é a única bijeção crescente em um conjunto finito. Isso implica em $f$ igual à identidade em $\operatorname{Fix}(g)$, portanto, $f$ fixa todos os pontos de $\operatorname{Fix}(g)$.

Como $f \in G$ é um elemento não-trivial qualquer, temos que os pontos $x_{1}, x_{2}, \ldots, x_{k}$ são pontos fixos globais. E como $g \in G$ não-trivial, foi escolhida de forma arbitrária, temos que todos os pontos fixos são globais.

Definição 2.2.29 Seja $\phi: G \times X \rightarrow X$ uma ação de grupos. Um subconjunto $Y \subset X$ é chamado de $G$-invariante, se para toda $g \in G$ e todo $y \in Y$ segue que $\phi(g, y) \in Y$.

Observação 2.2.30 Dados uma ação de grupos $\phi: G \times X \rightarrow X$ e $Y \subset X$ subconjunto $G$-invariante, a restrição de $\phi$ a $G \times Y$ define uma ação de grupos $\phi: G \times Y \rightarrow Y$.

Definição 2.2.31 Dados $(G, \star) e(F, \circ)$ dois grupos, uma aplicação $h: G \rightarrow F$ é um homomorfismo se para todo $g_{1}, g_{2} \in G$ se verifica que

$$
h\left(g_{1} \star g_{2}\right)=h\left(g_{1}\right) \circ h\left(g_{2}\right) .
$$

Além disso, se a aplicação for bijetiva, dizemos que ela é um isomorfismo. Por fim, se $(G, \star)=(F, \circ)$ então um isomorfismo será chamado de automorfismo.

Lema 2.2.32 Automorfismos contínuos de $(\mathbb{R},+)$ são homotetias, ou seja, são da forma:

$$
x \mapsto a . x \text {, onde } a \in \mathbb{R}, \text { com } a \neq 0 .
$$


Prova. Um automorfismo $h$ de $(\mathbb{R},+)$ deve satisfazer

$$
h(a+b)=h(a)+h(b) \text { para todo } a, b \in \mathbb{R} .
$$

Segue então, que $h(0)=0$ e que $-h(1)=h(-1)$. Por indução, é fácil mostrar que $h(k)=h(1) . k$, para todo $k \in \mathbb{Z}$. Daí segue que $h(r)=h(1) . r$, para todo $r \in \mathbb{Q}$. Por continuidade, concluimos que $h(x)=h(1) . x$, para todo $x \in \mathbb{R}$. E como é uma bijeção, $h(1) \neq h(0)=0$. Assim se prova o lema.

Observação 2.2.33 Segue diretamente da Compatibilidade de ações de grupos que uma ação $\phi: G \rightarrow \operatorname{Bij}(X)$ é um homomorfismo do grupo $(G, \star)$ para o grupo $(\operatorname{Bij}(X), \circ)$, onde o é a composição de funções.

Lema 2.2.34 Sejam $G \subset$ Homeo $_{+}(\mathbb{R})$ uma ação de grupos e $F \subset \mathbb{R}$ um subconjunto $G$-invariante, fechado e sem pontos isolados.

Seja $h: \mathbb{R} \rightarrow \mathbb{R}$ uma função continua, crescente e sobrejetiva, com $h$ constante nas componentes do complementar de $F$ e injetiva em $F \backslash \overline{F^{c}}$.

Então, $\phi: G \rightarrow$ Homeo $_{+}(\mathbb{R})$ definida por $\phi(g):=h \circ g \circ h^{-1}$ é um homomorfismo de grupos.

Prova. Seja $F^{c}=\bigsqcup_{n \in \mathbb{N}}\left(a_{n}, b_{n}\right)$ o complementar de $F$ (onde os intervalos são disjuntos dois a dois).

Afirmação. $\quad F \backslash \overline{F^{c}}$ e $F \cap \overline{F^{c}}$ são $G$-invariantes.

Prova. Como $F$ é $G$-invariante, temos diretamente que $F^{c}$ também é $G$ invariante. Mas $G$ é grupo de homeomorfismos, implica em $\overline{F^{c}} G$-invariante. Assim, $\left(\overline{F^{c}}\right)^{c}$ também é $G$-invariante. Portanto, $F \backslash \overline{F^{c}}$ e $F \cap \overline{F^{c}}$ são $G$ invariantes.

Afirmação. Para todo $g \in G, \phi(g): \mathbb{R} \rightarrow \mathbb{R}$ é um homeomorfismo crescente.

Prova. Em primeiro lugar veremos que para todo $g \in G, \phi(g): \mathbb{R} \rightarrow \mathbb{R}$ é uma função bem definida. Para isso, é necessário mostrar que:

Para todo $x \in \mathbb{R}, \phi(g)(x) \subset \mathbb{R}$ é exatamente um ponto.

Temos que considerar os seguintes dois casos:

Caso $h(x) \in h\left(F \backslash \overline{F^{c}}\right)$ : Neste caso, $h^{-1}(h(x))$ é exatamente um ponto. E como $h \circ g$ é função, temos: $h \circ g\left(h^{-1}(h(x))\right)$ é um ponto, e assim, $\phi(g)(h(x))$ é exatamente um ponto. 
Caso $h(x) \in h\left(\overline{F^{c}}\right)$ : Então, $h(x) \in h\left(\left[a_{n}, b_{n}\right]\right)$. Temos pela definição de $h$, que

$$
h^{-1}(h(x))=\left[a_{n}, b_{n}\right] .
$$

Como $g$ é um homeomorfismo crescente, então

$$
g\left(h^{-1}(h(x))\right)=g\left(\left[a_{n}, b_{n}\right]\right)=\left[g\left(a_{n}\right), g\left(b_{n}\right)\right] .
$$

Mas, $F \cap \overline{F^{c}}=\bigcup_{n \in \mathbb{N}}\left\{a_{n}, b_{n}\right\}$ é $G$-invariante. Logo, existe $m \in \mathbb{N}$, com

$$
\left[g\left(a_{n}\right), g\left(b_{n}\right)\right]=\left[a_{m}, b_{m}\right]
$$

Portanto,

$$
\phi(g)(h(x))=h \circ g\left(h^{-1}(h(x))\right)=h\left(\left[a_{m}, b_{m}\right]\right) \text { é um único ponto. }
$$

Concluímos que para todo $x \in \mathbb{R}, \phi(g)(h(x))$ é exatamente um ponto. A primeira parte da afirmação segue observando que $h$ é sobrejetiva.

Para ver que $\phi(g)$ é um homeomorfismo observamos que para todo $g \in G$, $\phi(g)$ é crescente. Com efeito, tome $x_{1}<x_{2}$. Temos:

$$
\begin{gathered}
y_{1}<y_{2}, \text { para todo } y_{1} \in h^{-1}\left(x_{1}\right), y_{2} \in h^{-1}\left(x_{2}\right) \\
\Rightarrow h \circ g\left(y_{1}\right) \leq h \circ g\left(y_{2}\right), \text { para todo } y_{1} \in h^{-1}\left(x_{1}\right), y_{2} \in h^{-1}\left(x_{2}\right) \\
\Rightarrow \phi(g)\left(x_{1}\right) \leq \phi(g)\left(x_{2}\right) .
\end{gathered}
$$

Porém, $h \circ g\left(y_{1}\right)=h \circ g\left(y_{2}\right)$, só se, existe $n \in \mathbb{N}$, com

$$
g\left(y_{1}\right), g\left(y_{2}\right) \in\left[a_{n}, b_{n}\right] \Rightarrow y_{1}, y_{2} \in\left[g^{-1}\left(a_{n}\right), g^{-1}\left(b_{n}\right)\right]=\left[a_{m}, b_{m}\right]
$$

O que implicaria em: $h\left(y_{1}\right)=h\left(y_{2}\right)$. Absurdo, pois $h\left(y_{1}\right)=x_{1}<x_{2}=h\left(y_{2}\right)$. Portanto, $\phi(g)$ é injetiva.

Além disso, $\phi(g)$ é sobrejetiva. Com efeito, tome $y \in \mathbb{R}$. Observe que $h^{-1}(y)$ é não-vazio pois $h$ é sobrejetiva, e portanto, $h \circ g^{-1}\left(h^{-1}(y)\right)$ é não-vazio também, pois $g$ é homeomorfismo. Assim:

$$
\begin{aligned}
& g^{-1}\left(h^{-1}(y)\right) \in h^{-1}\left(h \circ g^{-1}\left(h^{-1}(y)\right)\right) \\
& \Rightarrow h^{-1}(y) \in g \circ h^{-1}\left(h \circ g^{-1}\left(h^{-1}(y)\right)\right) \\
& \Rightarrow y \in h \circ g \circ h^{-1}\left(h \circ g^{-1}\left(h^{-1}(y)\right)\right) .
\end{aligned}
$$

Ou seja, $y \in \phi(g)\left(h \circ g^{-1}\left(h^{-1}(y)\right)\right)$, portanto $h \circ g^{-1}\left(h^{-1}(y)\right)$ é não-vazio, implica 
que existe $x \in \mathbb{R}$ tal que: $\phi(g)(x)=y$.

Portanto, para toda $g \in G, \phi(g)$ é crescente e bijetiva, logo pelo Lema 2.1.13, é homeomorfismo crescente.

Afirmação. A aplicação $\phi: G \rightarrow \operatorname{Homeo}_{+}(\mathbb{R})$ é um homomorfismo.

Prova. Basta mostrar que $\phi\left(g_{1} \circ g_{2}\right)(x)=\phi\left(g_{1}\right) \circ \phi\left(g_{2}\right)(x)$, para todo $x \in \mathbb{R}$. O resultado é direto da definição de $\phi$ para $h(x) \in h\left(F \backslash \overline{F^{c}}\right)$, pois $h$ é bijetiva.

Agora, considere $h(x) \in h\left(\overline{F^{c}}\right)$. Existe $n \in \mathbb{N}$, com $h^{-1}(h(x))=\left[a_{n}, b_{n}\right] \mathrm{e}$ $g_{2}\left(\left[a_{n}, b_{n}\right]\right)=\left[a_{m}, b_{m}\right]$. Lembrando que $h^{-1} \circ h\left(\left[a_{m}, b_{m}\right]\right)=\left[a_{m}, b_{m}\right]$. Temos:

$$
\begin{gathered}
\phi\left(g_{1}\right) \circ \phi\left(g_{2}\right)(h(x))=\phi\left(g_{1}\right) \circ h \circ g_{2} \circ h^{-1}(h(x))=\phi\left(g_{1}\right) \circ h \circ g_{2}\left(\left[a_{n}, b_{n}\right]\right) \\
=h \circ g_{1} \circ h^{-1} \circ h\left(\left[a_{m}, b_{m}\right]\right)=h \circ g_{1}\left(\left[a_{m}, b_{m}\right]\right)=h \circ g_{1} \circ g_{2}\left(\left[a_{n}, b_{n}\right]\right) \\
=h \circ g_{1} \circ g_{2} \circ h^{-1}(h(x))=\phi\left(g_{1} \circ g_{2}\right)(h(x)),
\end{gathered}
$$

provando a afirmação.

Concluímos assim, a prova do lema.

Definição 2.2.35 Sejam $\phi_{1}: G \times X \rightarrow X$ e $\phi_{2}: F \times Y \rightarrow Y$ duas ações de grupos fiéis e $h: X \rightarrow Y$ aplicação bijetiva. Dizemos que $\phi_{1}$ e $\phi_{2}$ são conjugados por $h$ se existe bijeção $\rho: G \rightarrow F$, tal que para todo $g \in G$ e todo $x \in X$ se verifica

$$
h\left(\phi_{1}(g, x)\right)=\phi_{2}(\rho(g), h(x)) .
$$

Ou seja, o diagrama comuta:

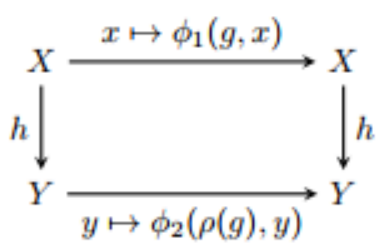

Observação 2.2.36 Sejam $X, Y$ dois espaços topológicos homeomorfos e $G$ um grupo que age sobre $X$. Sejam $\phi$ a ação associada e $h$ o homeomorfismo entre os dois espaços. Podemos definir uma ação $\phi^{\prime}$ do grupo G agindo em $Y$ conjugando $\phi$ por $h$, isto é:

$$
\phi^{\prime}(g, y):=h\left(\phi\left(g, h^{-1}(y)\right)\right)
$$


Definição 2.2.37 Sejam $\phi_{1}: G \times X \rightarrow X$ e $\phi_{2}: F \times Y \rightarrow Y$ duas ações de grupos fiéis e $h: X \rightarrow Y$ uma aplicação sobrejetiva. Dizemos que $\phi_{1}$ é semiconjugada à $\phi_{2}$ por $h$ se existe aplicação $\rho: G \rightarrow F$, tal que para todo $g \in G$ e todo $x \in X$ se verifica

$$
h\left(\phi_{1}(g, x)\right)=\phi_{2}(\rho(g), h(x)) .
$$

Ou seja, o diagrama comuta:

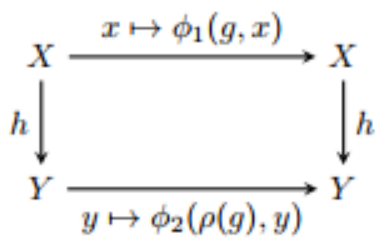

Observação 2.2.38 A aplicação $\rho: G \rightarrow F$ das Definições 2.3.35 e 2.3.37 é um homomorfismo de grupos. Com efeito, como $\phi_{1}, \phi_{2}$ são ações de grupos satisfazendo, para todo $g \in G$ e todo $x \in X$

$$
h\left(\phi_{1}(g, x)\right)=\phi_{2}(\rho(g), h(x)),
$$

segue que para todo $g_{1}, g_{2} \in G$ e todo $x \in X$

$$
\phi_{2}\left(\rho\left(g_{1}\right), h\left(\phi_{1}\left(g_{2}, x\right)\right)\right)=\phi_{2}\left(\rho\left(g_{1}\right), \phi_{2}\left(\rho\left(g_{2}\right), h(x)\right)\right)=\phi_{2}\left(\rho\left(g_{1}\right) \circ \rho\left(g_{2}\right), h(x)\right) .
$$

Por outro lado,

$\phi_{2}\left(\rho\left(g_{1}\right), h\left(\phi_{1}\left(g_{2}, x\right)\right)\right)=h\left(\phi_{1}\left(g_{1}, \phi_{1}\left(g_{2}, x\right)\right)\right)=h\left(\phi_{1}\left(g_{1} \circ g_{2}, x\right)\right)=\phi_{2}\left(\rho\left(g_{1} \circ g_{2}\right), h(x)\right)$.

Portanto, temos:

$$
\phi_{2}\left(\rho\left(g_{1} \circ g_{2}\right), h(x)\right)=\phi_{2}\left(\rho\left(g_{1}\right) \circ \rho\left(g_{2}\right), h(x)\right) .
$$

Como $h: X \rightarrow Y$ é sobrejetiva, segue para todo $g_{1}, g_{2} \in G$ e todo $y \in Y$

$$
\phi_{2}\left(\rho\left(g_{1} \circ g_{2}\right), y\right)=\phi_{2}\left(\rho\left(g_{1}\right) \circ \rho\left(g_{2}\right), y\right) .
$$

Por fim, como $\phi_{2}$ é ação fiel, temos para todo $g_{1}, g_{2} \in G$

$$
\rho\left(g_{1} \circ g_{2}\right)=\rho\left(g_{1}\right) \circ \rho\left(g_{2}\right) \text {. }
$$

Logo, a aplicação $\rho: G \rightarrow F$ é homomorfismo de grupos. 


\section{3}

\section{Conjuntos minimais de $\mathbb{R}$}

Um conjunto minimal é uma propriedade dinâmica de uma ação de grupos, porém, de forma surpreendente, a existência e topologia desses conjuntos implicam em restrições algébricas no grupo, criando uma conexão entre dinâmica e álgebra.

Nessa seção vamos definir e classificar todos os possíveis conjuntos minimais de $\mathbb{R}$. Essa classificação será essencial para os próximos capítulos.

\subsection{1}

\section{Definição e classificação dos conjuntos minimais de $\mathbb{R}$}

Definição 2.3.1 Seja $G \subset \operatorname{Homeo}(\mathbb{R})$ uma ação de grupo sobre $\mathbb{R}$. Um conjunto não-vazio $K \subset \mathbb{R}$ é dito minimal, se verifica:

- K é invariante por $G$, i.e., para todo $g \in G$ temos $g(K)=K$,

- Ké fechado,

- K é minimo por inclusão, i.e., se $K^{\prime} \subset K$ não-vazio é fechado e invariante, então $K^{\prime}=K$.

Lema 2.3.2 Um conjunto $K$ é minimal se, e só se, para todo $x \in K$, o fecho da órbita de $x$ é igual a $K$, isto é,

$$
\overline{G . x}=K, \text { para todo } x \in K \text {. }
$$

Prova. Para a implicação $\Rightarrow$. Tome $x \in K$, como $K$ é um conjunto fechado e $G$-invariante, temos que

$$
\overline{G . x} \subset K .
$$

Porém, $\overline{G . x}$ é também um fechado $G$-invariante. Portanto, pela minimalidade de $K$, segue que $\overline{G \cdot x}=K$.

Agora, para $\Leftarrow$. Comece observando que se para todo $x \in K$ o fecho da órbita G.x pertence a $K$, em particular, para todo $x \in K$ a órbita $G . x \subset K$. Portanto, $K$ é $G$-invariante.

Além disso, $K$ é fechado. Basta tomar $x \in K$, por definição de fecho segue que $\overline{G . x}$ é fechado. E como $\overline{G . x}=K$, segue que $K$ é fechado.

Por fim, $K$ é minimo por inclusão. Com efeito, tome $K^{\prime} \subset K$ não-vazio, fechado e $G$-invariante. Tome $y \in K^{\prime}$, note que por ser fechado $G$-invariante, segue que $\overline{G . y} \subset K^{\prime}$. Porém $y \in K^{\prime} \subset K$ implica em $\overline{G . y}=K$, ou seja, $K \subset K^{\prime}$.

Portanto, concluímos que $K$ é conjunto minimal. 
Definição 2.3.3 Um subconjunto $C \subset \mathbb{R}$ é do tipo Cantor bi-ilimitado se:

- C é perfeito, i.e., fechado e sem pontos isolados,

- C é bi-ilimitado, i.e., ilimitado inferiormente e superiormente,

- C é totalmente desconexo, i.e., não contém nenhum intervalo.

Teorema 2.3.4 (Conjuntos minimais da reta) Seja $G \subset \mathrm{Homeo}_{+}(\mathbb{R})$ com um conjunto minimal $K$. Então, a aderência de toda órbita contém um conjunto minimal. E além disso, temos as seguintes possibilidades disjuntas:

(1) Todas as órbitas são densas em $\mathbb{R}$ e $K=\mathbb{R}$ é o único conjunto minimal.

(2) Todos os conjuntos minimais são pontos fixos globais da ação de $G$.

(3) Todos os conjuntos minimais são órbitas fechadas de $G$, que são conjuntos discretos bi-ilimitados. Além disso, existe $g \in G$ sem pontos fixos tal que todos os conjuntos minimais são órbitas de $g$.

(4) Existe um único conjunto minimal $K$ que é um conjunto do tipo Cantor bi-ilimitado. Além disso, $K$ está contido na aderência de todas as órbitas.

Prova. Seja $K \subset \mathbb{R}$ um conjunto minimal da ação de $G$.

(1) Se $K=\mathbb{R}$, então pelo Lema 2.3.2, toda órbita será densa em $\mathbb{R}$ e assim, $K$ é o único minimal da ação. Isso prova o primeiro caso do teorema.

(2) Veremos que o caso (2) ocorre quando existe algum conjunto minimal limitado superiormente ou inferiormente.

Afirmação. Se $K$ possui cota superior ou inferior, então $K$ é um ponto fixo global da ação. Além disso, todos os conjuntos minimais da ação de $G$ são pontos fixos globais.

Prova. Suponha que $K$ possui cota superior, defina

$$
x_{0}:=\sup x \in K \text {. }
$$

Note que $x_{0} \in K$, pois $K$ é fechado. Veremos que $g\left(x_{0}\right)=x_{0}$ para todo $g \in G$. Como $K$ é $G$-invariante: para todo $g \in G$, se verifica

$$
g\left(x_{0}\right) \in K \Rightarrow g\left(x_{0}\right) \leq x_{0} \Rightarrow x_{0} \leq g^{-1}\left(x_{0}\right) .
$$

Mas o mesmo resultado é válido para $g^{-1} \in G$, e assim:

$$
g^{-1}\left(x_{0}\right) \in K \Rightarrow g^{-1}\left(x_{0}\right) \leq x_{0} \Rightarrow g\left(x_{0}\right)=x_{0} .
$$


Portanto, $x_{0} \in K$ é um ponto fixo global, e então é um conjunto fechado $G$-invariante contido em $K$. Pela definição de minimal, temos $K=\left\{x_{0}\right\}$.

De forma análoga, temos se $K$ tem cota inferior, então $K$ é um ponto fixo global.

Por fim, considere um outro conjunto minimal $K_{2}$ para ação de $G$ diferente de $K$. Temos que $K$, por ser um ponto fixo global é cota superior ou inferior para $K_{2}$. E portanto, usando a afirmação anterior, $K_{2}$ também é um ponto fixo global da ação $G$. Assim, concluímos a afirmação e o segundo caso do teorema.

Em vista dos casos (1) e (2) a partir de agora temos que considerar ações cujos conjuntos minimais são bi-ilimitados e diferentes de $\mathbb{R}$.

(3) A prova desse caso será consequência de algumas afirmações, começando por:

Afirmação. Se $K$ possui um ponto isolado, então todos os pontos de $K$ são isolados.

Prova. Se $K$ possui um ponto isolado, então, o conjunto $K^{\prime}$, dos pontos de acumulação de $K$ :

$$
K^{\prime}:=\{x \in K \mid K \cap(V \backslash\{x\}) \neq \emptyset \text {, para toda vizinhança } V \text { de } x\}
$$

é um fechado invariante estritamente contido em $K$, logo deverá ser vazio. E portanto, todos os pontos de $K$ são pontos isolados.

Agora, considere $K$ um conjunto minimal com apenas pontos isolados e bi-ilimitado.

Afirmação. Existe $g \in G$ livre de pontos fixos, tal que $K$ é uma órbita fechada de $g$.

Prova. Como $K$ é fechado formado de pontos isolados, fica claro que podemos ordená-lo em forma crescente

$$
K:=\left\{x_{n} \mid n \in \mathbb{Z}, x_{n}<x_{n+1}\right\}
$$

Como a órbita de $x_{0}$ é densa em todo $K$ e $x_{1} \in K$ é ponto isolado, temos que existe $g \in G$, com $g\left(x_{0}\right)=x_{1}$. Porém, $g: \mathbb{R} \rightarrow \mathbb{R}$ é homeomorfismo crescente, e $K$ é $g$-invariante. Então $g$ restrito a $K$ deve ser uma bijeção crescente, respeitando $g\left(x_{0}\right)=x_{1}$. A única bijeção crescente possível é o shift: $g\left(x_{n}\right)=x_{n+1}$, para todo $n \in \mathbb{Z}$. E assim, $K=\bigcup_{n \in \mathbb{Z}}\left\{g^{n}\left(x_{0}\right)\right\}$, ou seja, $K$ é uma órbita fechada de $g$. 
Além disso, como $g\left(x_{n}\right)=x_{n+1}$, para todo $n \in \mathbb{Z}$, fica claro que $g: \mathbb{R} \rightarrow \mathbb{R}$ é livre de pontos fixos.

Agora considere $K_{2}$, um segundo conjunto minimal para a ação de $G$, diferente de $K$. É claro que $K_{2} \cap K=\emptyset$. Caso contrário, a interseção seria um fechado invariante menor. Portanto, existe $n \in \mathbb{Z}$ com:

$$
K_{2} \cap\left(x_{n}, x_{n+1}\right) \neq \emptyset .
$$

E como $K_{2}$ é $g$-invariante, temos que:

$$
K_{2} \cap\left(x_{i}, x_{i+1}\right) \neq \emptyset \text {, para todo } i \in \mathbb{Z} \text {. }
$$

Agora defina o estabilizador $H \subset G$ do ponto $x_{0}$ :

$$
H:=\left\{g \in G \mid g\left(x_{0}\right)=x_{0}\right\}
$$

Afirmação. Seja $h \in G \operatorname{com} h\left(x_{n}\right)=x_{n}$, para algum $n \in \mathbb{Z}$. Então, $h \in H$. Prova. Note que $h: \mathbb{R} \rightarrow \mathbb{R}$ define uma bijeção crescente em $K=\left\{x_{m} \mid m \in\right.$ $\left.\mathbb{Z}, x_{m}<x_{m+1}\right\}$, e como $h\left(x_{n}\right)=x_{n}$, temos que $h\left(x_{m}\right)=x_{m}$, para todo $m \in \mathbb{Z}$. Em particular, $h\left(x_{0}\right)=x_{0}$ o que implica em $h \in H$.

Afirmação. $K_{2}$ também é bi-ilimitado e formado apenas por pontos isolados. Além disso, $K_{2}$ tem exatamente um ponto em cada intervalo $\left[x_{n}, x_{n+1}\right]$, para todo $n \in \mathbb{N}$.

Prova. O fato de $K_{2}$ ser bi-ilimitado decorre dos casos anteriores. Defina,

$$
Y_{n}:=K_{2} \cap\left[x_{n}, x_{n+1}\right] .
$$

Note que $Y_{n}$ é fechado e $H$-invariante. Além disso, $Y_{n}$ é minimal, pela minimalidade de $K_{2}$. Portanto, $Y_{n}$ é um conjunto minimal para ação de $H$, e é cotado superiormente por $x_{n+1}$. Logo, pelo caso (2), $Y_{n}=\left\{x_{n}^{\prime}\right\}$ é um ponto fixo global de $H$, contido em $\left(x_{n}, x_{n+1}\right)$.

Por outro lado,

$$
\bigcup_{n \in \mathbb{Z}} Y_{n}=\bigcup_{n \in \mathbb{Z}} K_{2} \cap\left[x_{n}, x_{n+1}\right]=K_{2} \cap \mathbb{R}=K_{2}
$$

Ou seja,

$$
K_{2}=\left\{x_{n}^{\prime} \mid n \in \mathbb{Z}, x_{n}^{\prime}<x_{n+1}^{\prime}\right\}
$$

Portanto, $K_{2}$ é formado apenas por pontos isolados. 
Para concluir a prova do caso (3), note que $g^{n}\left(x_{0}^{\prime}\right) \in K_{2}$, pois $K_{2}$ é $G$-invariante. E como $g$ é crescente, segue que

$$
x_{0}^{\prime} \in\left[x_{0}, x_{1}\right] \text { e } g^{n}\left(\left[x_{0}, x_{1}\right]\right)=\left[x_{n}, x_{n+1}\right] \Rightarrow g^{n}\left(x_{0}^{\prime}\right) \in\left[x_{n}, x_{n+1}\right]
$$

Portanto,

$$
g^{n}\left(x_{0}^{\prime}\right) \in K_{2} \cap\left[x_{n}, x_{n+1}\right]=Y_{n}=\left\{x_{n}^{\prime}\right\}
$$

então, fica claro que $K_{2}=\bigcup_{n \in \mathbb{Z}} g^{n}\left(x_{0}^{\prime}\right)$, ou seja, $K_{2}$ é uma órbita de $g$. Concluindo assim, o terceiro caso do teorema.

(4) Novamente, a prova será dividida em afirmações.

Afirmação. Se $K$ é sem pontos isolados, bi-ilimitado e diferente de $\mathbb{R}$, então $K$ é do tipo Cantor bi-ilimitado.

Prova. Como $K$ é fechado e sem pontos isolados, segue por definição que $K$ é perfeito.

Além disso, caso $K$ contenha um intervalo $(a, b)$, podemos escolher ele maximal com a propriedade da inclusão. Como $K$ é fechado, ele contém o fecho do intervalo $[a, b]$. Assim, o bordo de $K$ está estritamente contido em $K$ e é um fechado invariante da ação. O que contradiz a minimalidade do conjunto $K$. Portanto, $K$ não contém intervalos e assim é totalmente desconexo.

Concluindo, temos que $K$ é um conjunto perfeito, bi-ilimitado e totalmente desconexo, logo é do tipo Cantor bi-ilimitado.

Afirmação. A órbita de qualquer ponto de $\mathbb{R}$ é densa sobre $K$, ou seja, $K \subset \overline{G . x}$, para todo $x \in \mathbb{R}$.

Prova. Pelo Lema 2.3.2, os pontos de acumulação da órbita de qualquer ponto $x \in K$ é igual a $K$. Agora, tome $x \notin K$. Temos que existe $(a, b)$, uma componente conexa do complementar $\mathbb{R} \backslash K$, que contém $x$.

Como $a, b \in K$, existe uma sequência $\left\{g_{i}\right\}_{i \in \mathbb{N}} \subset G$, com $g_{i}(a) \rightarrow b$ quando $i \rightarrow+\infty$. A menos de tomar uma subsequência, vamos considerar $\left\{g_{i}(a)\right\}_{i \in \mathbb{N}}$ sequência monótona decrescente. Note que para todo $i \in \mathbb{N}$, temos

$$
g_{i}(b) \in\left[g_{i}(a), g_{i+1}(a)\right]
$$

portanto, $g_{i}(b) \rightarrow b$, e assim como $x \in(a, b)$, temos $g_{i}(x) \rightarrow b$. Como $b$ tem órbita densa em $K$, por continuidade o mesmo vai valer para $x$.

Concluímos assim que a órbita de qualquer ponto de $\mathbb{R}$ é densa sobre o conjunto minimal $K$.

Por fim, se $K_{2}$ é um segundo conjunto minimal para a ação de $G$, como a órbita dos pontos de $K_{2}$ são densas sobre $K$, e $K_{2}$ é fechado, temos: $K \subset K_{2}$. 
E assim, pela minimalidade de $K_{2}$, segue que $K_{2}=K$. Portanto, o minimal $K$ é único para a ação de $G$. O que conclui o quarto caso do teorema.

Provamos portanto que dada uma ação sobre $\mathbb{R}$, qualquer conjunto minimal da ação necessariamente entra em exatamente um dos casos listados. Concluindo assim, a prova do teorema.

\subsection{2}

\section{Critérios para existência de conjuntos minimais}

Não é verdade que toda ação sobre $\mathbb{R}$ possui um conjunto minimal, um contra-exemplo muito interessante pode ser visto em [Bo]. Portanto, neste capítulo estudaremos um critério para existência de conjuntos minimais que será muito útil nos próximos capítulos.

Lema 2.3.5 Se existe um segmento compacto $I \subset \mathbb{R}$, tal que toda órbita passa por $I$, i.e. para todo $x \in \mathbb{R}, G . x \cap I \neq \emptyset$, então a aderência de toda órbita contém um conjunto minimal.

Prova. Sem perda, podemos aumentar o intervalo $I$ fazendo com que todas as órbitas passem pelo interior do intervalo.

Dado $x \in \mathbb{R}$, considere $\mathcal{A}$ o conjunto dos compactos contidos em $\overline{G . x} \cap I$ que são interseção de um fechado $G$-invariante com o intervalo $I$, munido da inclusão $\subset$.

Observe que $(\mathcal{A}, \subset)$ é um conjunto parcialmente ordenado que satisfaz as hipóteses do Lema de Zorn. Portanto, existe elemento mínimo $Y$. Defina agora o menor conjunto invariante que contém $Y$ :

$$
K:=G \cdot Y=\bigcup_{y \in Y} G \cdot y .
$$

Afirmação. O conjunto $K$ é fechado.

Prova. Tome qualquer $k_{\infty} \in \bar{K}$, existe uma sequência $\left\{k_{n}\right\}_{n \in \mathbb{N}} \subset K$ que converge para $k_{\infty}$. Por hipótese, a órbita de $k_{\infty}$ passa pelo interior de $I$. Portanto, existe $g \in G$, tal que $g\left(k_{\infty}\right)$ está contido no interior de $I$. Assim, pela continuidade de $g: \mathbb{R} \rightarrow \mathbb{R}$, a sequência $\left\{g\left(k_{n}\right)\right\}_{n \in \mathbb{N}}$ converge para $g\left(k_{\infty}\right)$ e portanto, possui uma subsequência contida no intervalo, $\left\{g\left(k_{n_{i}}\right)\right\}_{i \in \mathbb{N}} \subset K \cap I$. Porém, pela definição de $Y$, temos: $K \cap I=Y$, pois $Y$ é a interseção de um conjunto invariante com o intervalo $I$, e $K$ é o menor conjunto invariante que contém $Y$. Então temos uma subsequência em $Y,\left\{g\left(k_{n_{i}}\right)\right\}_{i \in \mathbb{N}} \subset Y$, que converge para $g\left(k_{\infty}\right)$. Como $Y$ é fechado, temos $g\left(k_{\infty}\right) \in Y$. Assim, como $K$ é 
$G$-invariante e contém $Y$, temos que

$$
g^{-1}\left(g\left(k_{\infty}\right)\right)=k_{\infty} \in K
$$

Assim, $k_{\infty} \in K$. Como isto vale para todo ponto de acumulação de $K$, temos $\bar{K} \subset K$. Logo, $K$ é fechado.

Falta por provar a minimaidade de $K$. Para isso, suponha $K^{\prime} \subset K$ fechado invariante. Note que:

$$
K^{\prime} \cap I \subset K \cap I=Y .
$$

Pela minimalidade de $Y$, temos que: $K^{\prime} \cap I=Y$. Portanto, $Y \subset K^{\prime}$.

Agora, tome $k \in K$. Pela definição, existem $g \in G$ e $y \in Y \operatorname{com} g(y)=k$. Mas $y \in K^{\prime}$ e $K^{\prime}$ é $G$-invariante, assim

$$
k=g(y) \in K^{\prime} \Rightarrow K \subset K^{\prime},
$$

portanto $K=K^{\prime}$. E assim $K$ é um conjunto minimal contido no fecho da órbita de $x, \overline{G . x}$.

Corolário 2.3.6 Se existe uma famillia $g_{1}, g_{2}, \ldots, g_{n} \in G$, com $n \geq 2$, sem pontos fixos em comum, então a aderência de toda órbita contém um conjunto minimal.

Prova. Defina, para todo $x \in \mathbb{R}$, a função:

$$
F(x):=\max _{i \in\{1, \ldots, n\}}\left\{g_{i}^{ \pm 1}(x)\right\}
$$

A função $F: \mathbb{R} \rightarrow \mathbb{R}$ é crescente, contínua e $F(x)>x$ para todo $x \in \mathbb{R}$, pois estamos tomando tanto $g_{i}$ quanto $g_{i}^{-1}$ na definição da $F$, e não existem pontos fixos comuns a toda a família. Assim, temos pelo Lema 2.2.14,

$$
\begin{gathered}
\lim _{n \rightarrow+\infty} F^{n}(x)=+\infty, \text { para todo } x \in \mathbb{R}, \\
\lim _{n \rightarrow+\infty} F^{-n}(x)=-\infty, \text { para todo } x \in \mathbb{R} .
\end{gathered}
$$

Defina agora o intervalo compacto:

$$
I:=[0, F(0)]
$$

Vamos mostrar que todas as órbitas intersectam o intervalo $I$ :

Como $\lim _{n \rightarrow+\infty} F^{-n}(x)=-\infty$, segue que para todo $x \in \mathbb{R}$ existem $y<0$ e $k \in \mathbb{N} \operatorname{com} F^{-k}(x)=y$, e como $\lim _{n \rightarrow+\infty} F^{n}(y)=+\infty$, segue que existe $n \in \mathbb{N}$, 
tal que $F^{n}(y)>F(0)$. Seja então, $m \in\{1,2, \ldots, n\}$, o primeiro inteiro positivo, tal que $F^{m}(y)>F(0)$.

Como $F$ é crescente, para todo $x<0$, temos $F(x)<F(0)$. Portanto, como $F^{m}(y)>F(0)$, segue que $F^{m-1}(y)>0$. Por outro lado, como $m$ é o primeiro inteiro, tal que $F^{m}(y)>F(0)$, segue que $F^{m-1}(y) \leq F(0)$. Portanto, $F^{m-1}(y) \in(0, F(0)] \subset I$, e assim, $F^{m-1-k}(x) \in I$. Assim, para todo $x \in \mathbb{R}$, a sequência $\left\{F^{n}(x)\right\}_{n \in \mathbb{Z}}$ passa por $I$. E como $F^{n}(x) \in G$.x, para todo $n \in \mathbb{Z}$, concluímos que:

Para todo $x \in \mathbb{R}, G . x \cap I \neq \emptyset$.

Logo, o Lema 2.3.5 se aplica. 


\section{Ações de grupos com até um ponto fixo}

Neste capítulo nosso objetivo é apresentar e demonstrar os Teoremas de Hölder e Solodov, que classificam a menos de semi-conjugação os grupos de homeomorfismos com no máximo um ponto fixo.

No decorrer das demonstrações veremos que essa hipótese dinâmica implica na existência de uma relação de ordem no grupo, e como consequência restringe sua estrutura algébrica.

\section{1}

\section{Ações sem pontos fixos}

Começando com a hipótese de que nenhum elemento diferente da identidade possui pontos fixos, vamos ter como consequência imediata uma relação de ordem arquimediana, e com ela definiremos o número de translação como um morfismo de grupos. Este morfismo implicará na comutatividade do grupo (Corolário 3.1.6) e na semi-conjugação com um grupo de translações de $\mathbb{R}$ (Corolários 3.1.7 e 3.1.8), que são duas conclusões surpreendentes vindas de uma hipótese dinâmica.

Teorema 3.1.1 (Teorema de Hölder) Seja $G \subset \mathrm{Homeo}_{+}(\mathbb{R})$ um subgrupo livre de pontos fixos. Então, $G$ é um grupo abeliano, ordenado e arquimediano.

Além disso, existe um morfismo injetivo $t: G \rightarrow \mathbb{R}$ e uma aplicação continua crescente $h$ de $\mathbb{R}$, tais que para todo $g \in G$ e todo $x \in \mathbb{R}$ se verifica

$$
h \circ g(x)=h(x)+t(g)
$$

Ou seja, o diagrama abaixo comuta:

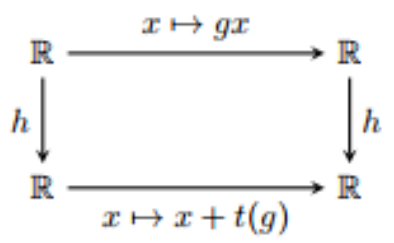

Dizemos que $t(g)$ é o número de translação de $g$. 
Prova. Dados $f, g \in G$, defina a seguinte relação,

$$
f \prec g \text {, se existe } x \in \mathbb{R} \text {, tal que } f(x)<g(x) \text {. }
$$

Lema 3.1.2 A relação $\prec$ define uma relação de ordem total sobre G. Além disso, ela é invariante por multiplicação à direita e à esquerda, pelos elementos de G. Finalmente, a ordem $\prec$ é arquimediana (Definição 2.2.5).

Prova. A prova do lema será consequência de uma série de afirmações simples.

Afirmação. Se existe $x_{0} \in \mathbb{R}$, com $f\left(x_{0}\right)<g\left(x_{0}\right)$, então para todo $x \in \mathbb{R}$, temos que $f(x)<g(x)$.

Prova. Suponha por absurdo o contrário. Assim, existe $x \in \mathbb{R} \operatorname{com} f(x) \geq g(x)$ e pelo Lema 2.1.9, existe $x_{1} \in \mathbb{R}$, com $f\left(x_{1}\right)=g\left(x_{1}\right)$ e então, $g^{-1} \circ f\left(x_{1}\right)=x_{1}$. Mas como $G$ é ação livre de pontos fixos, temos que: $g^{-1} \circ f=I d$, e assim, $f=g$. Absurdo, pois $f\left(x_{0}\right)<g\left(x_{0}\right)$.

Afirmação. $\prec$ é uma ordem total estrita.

Prova. Basta verificar cada um dos itens abaixo, que são diretos da afirmação anterior:

- Transitividade: Se $f \prec g$ e $g \prec h$, então, para todo $x \in \mathbb{R}$, temos: $f(x)<g(x)<h(x)$. E portanto, $f \prec h$.

- Assimetria: Se $f \prec g$, então, para todo $x \in \mathbb{R}$, temos: $f(x)<g(x)$. E portanto, é claro que $g \nprec f$.

- Irreflexividade: Pela definição de $\prec$, está claro que $f \nprec f$.

- Totalidade: Para todo $f, g \in G$, temos 3 possibilidades: $f(0)<g(0)$ e então $f \prec g$, ou $f(0)>g(0)$ e então $g \prec f$, ou $f(0)=g(0)$ e então $f=g$.

Assim, a afirmação está provada.

Afirmação. Para todo $f, g, h \in G$, temos:

- $f \prec g \Rightarrow h \circ f \prec h \circ g$ (invariante por multiplicação à esquerda),

- $f \prec g \Rightarrow f \circ h \prec g \circ h$ (invariante por multiplicação à direita).

Prova. Se $f \prec g$, então, temos que $f(x)<g(x)$, para todo $x \in \mathbb{R}$. Como $h \in G$ é crescente, temos que $h(f(x))<h(g(x))$, para todo $x$. E assim, $h \circ f \prec h \circ g$.

Por outro lado, como $h: \mathbb{R} \rightarrow \mathbb{R}$ é homeomorfismo, se $f(x)<g(x)$ para todo $x \in \mathbb{R}$, então temos que $f(h(y))<g(h(y))$, onde $y=h^{-1}(x)$. Ou seja, $f(h(y))<g(h(y))$ para todo $y \in \mathbb{R}$. E então, $f \circ h \prec g \circ h$. 
Afirmação. A ordem $\prec$ é arquimediana.

Prova. É nessessário mostrar que para todo $f, g \in G$ tal que $f \succ I d$ existe $n \in \mathbb{N} \operatorname{com} f^{n} \succ g$.

Porém, por definição de $f \succ I d$, temos $f(x)>x$ para todo $x \in \mathbb{R}$. Assim pelo Lema 2.2.14, fixando um $x_{0} \in \mathbb{R}$ temos que: $\lim _{n \rightarrow \infty} f^{n}\left(x_{0}\right)=+\infty$. E assim, existe $n \in \mathbb{N}$, com $f^{n}\left(x_{0}\right)>g\left(x_{0}\right), \log 0 f^{n} \succ g$.

Assim, temos uma relação de ordem total $\prec$ sobre $G$, invariante pela multiplicação à direita e à esquerda por elementos de $G$ e arquimediana. Concluindo assim a prova do lema.

Agora, fixando $f \in G$, tal que $f \succ I d$, defina para cada $n \in \mathbb{N}$ e cada $g \in G$ o número racional

$$
t_{f, n}(g):=\frac{p}{n}, \text { tal que: } f^{p} \preceq g^{n} \prec f^{p+1}
$$

que está bem definido, pois a relação $\prec$ é arquimediana (vide Lema 3.1.2).

Lema 3.1.3 A sequência $\left\{t_{f, n}(g)\right\}_{n \in \mathbb{N}}$ converge quando $n \rightarrow \infty$. Denotaremos o limite como $t_{f}(g)$.

Prova. Comece observando que pela invariância por multiplicação à direita e à esquerda, temos que se $f \prec g$ e $h \prec k$, então:

$$
f \circ h \prec g \circ h \prec g \circ k \text {. }
$$

Em particular, se $f \prec g$ podemos mostrar por indução que para todo $n>0$ se verifica $f^{n} \prec g^{n}$. Assim, $f^{p} \preceq g^{n} \prec f^{p+1}$ implica que para todo $k>0$,

$$
f^{p k} \preceq g^{n k} \prec f^{(p+1) k}
$$

e então $t_{f, n k}(g) \in\left[t_{f, n}(g), t_{f, n}(g)+\frac{1}{n}\right)$. Em particular, para todos $n, m$ positivos, temos:

$$
t_{f, n m}(g) \in\left[t_{f, n}(g), t_{f, n}(g)+\frac{1}{n}\right) \cap\left[t_{f, m}(g), t_{f, m}(g)+\frac{1}{m}\right) .
$$

Portanto, $\left[t_{f, n}(g), t_{f, n}(g)+\frac{1}{n}\right) \cap\left[t_{f, m}(g), t_{f, m}(g)+\frac{1}{m}\right) \neq \emptyset$ e então

$$
\left|t_{f, n}(g)-t_{f, m}(g)\right|<\sup \left\{\frac{1}{n}, \frac{1}{m}\right\}
$$

Logo, a sequência $\left\{t_{f, n}(g)\right\}_{n \in \mathbb{N}}$ é Cauchy e então convergente.

O limite $t_{f}(g)$ será denominado número de translação relativa de $g$ referente $\grave{a} f$.

Lema 3.1.4 Para cada $f \in G$, com $f \succ I d$, a aplicação $(G, \circ, \prec) \rightarrow(\mathbb{R},+,<)$, definida por $g \mapsto t_{f}(g)$ é um homomorfismo de grupos. 
Prova. Considere $g, h \in G$ e as composições $g \circ h$ e $h \circ g$. A menos de uma troca de nomes, podemos supor $g \circ h \preceq h \circ g$.

Afirmação. Para todo $n>0$, temos:

$-g \circ h^{n} \preceq h^{n} \circ g$.

$-g^{n} \circ h \preceq h \circ g^{n}$.

$-g^{n} \circ h^{n} \preceq(g \circ h)^{n} \preceq h^{n} \circ g^{n}$.

Prova. Vamos demonstrar por indução em $n$. Para os três itens, o caso $n=1$ é nossa hipótese dada por $g \circ h \preceq h \circ g$. Suponhamos verdadeiro para $n$ e olharemos para $n+1$ :

$$
\begin{aligned}
& g \circ h^{n+1}=\left(g \circ h^{n}\right) \circ h \preceq\left(h^{n} \circ g\right) \circ h=h^{n} \circ(g \circ h) \preceq h^{n} \circ(h \circ g)=h^{n+1} \circ g . \\
& g^{n+1} \circ h=g \circ\left(g^{n} \circ h\right) \preceq g \circ\left(h \circ g^{n}\right)=(g \circ h) \circ g^{n} \preceq(h \circ g) \circ g^{n}=h \circ g^{n+1} . \\
& g^{n+1} \circ h^{n+1}=g^{n} \circ\left(g \circ h^{n}\right) \circ h \preceq g^{n} \circ\left(h^{n} \circ g\right) \circ h=g^{n} \circ h^{n} \circ g \circ h=(g \circ h)^{n} \circ(g \circ h)=(g \circ h)^{n+1} \\
& (g \circ h)^{n+1}=(g \circ h) \circ(g \circ h)^{n} \preceq(h \circ g) \circ\left(h^{n} \circ g^{n}\right)=h \circ\left(g \circ h^{n}\right) \circ g^{n} \preceq h \circ\left(h^{n} \circ g\right) \circ g^{n}=h^{n+1} \circ g^{n+1} .
\end{aligned}
$$

Isso conclui a prova da afirmação.

Agora, fixe $n>0$ qualquer. Temos pelas definições de $t_{f, n}(g)$ e $t_{f, n}(h)$ :

$$
f^{n t_{f, n}(g)} \preceq g^{n} \prec f^{n t_{f, n}(g)+1} \text { e } f^{n t_{f, n}(h)} \preceq h^{n} \prec f^{n t_{f, n}(h)+1} \text {. }
$$

Compondo as desigualdades e utilizando a afirmação anterior, podemos deduzir:

$$
f^{n\left(t_{f, n}(g)+t_{f, n}(h)\right)} \preceq g^{n} \circ h^{n} \preceq(g \circ h)^{n} \preceq h^{n} \circ g^{n} \prec f^{n\left(t_{f, n}(g)+t_{f, n}(h)\right)+2} .
$$

Por outro lado, pela definição de $t_{f, n}(g \circ h)$, temos:

$$
f^{n t_{f, n}(g \circ h)} \preceq(g \circ h)^{n} \prec f^{n t_{f, n}(g \circ h)+1} .
$$

Deduzimos assim:

$$
t_{f, n}(g)+t_{f, n}(h) \leq t_{f, n}(g \circ h) \leq t_{f, n}(g)+t_{f, n}(h)+\frac{1}{n}
$$

Fazendo o limite quando $n \rightarrow \infty$, obtemos: $t_{f, n}(g)+t_{f, n}(h)=t_{f, n}(g \circ h)$.

Assim, a aplicação $(G, \circ, \prec) \rightarrow(\mathbb{R},+,<)$, definida por $g \mapsto t_{f}(g)$ é um homomorfismo de grupos.

Lema 3.1.5 O homomorfismo $g \mapsto t_{f}(g)$ é injetivo. 
Prova. Basta mostrar que se $g \neq I d$ então $t_{f}(g) \neq 0$. Como sempre, decomporemos a prova em diversas afirmações.

Afirmação. A aplicação $g \mapsto t_{f}(g)$ é crescente.

Prova. Precisamos provar que dados $g, h \in G$ com $g \preceq h$ se verifica $t_{f}(g) \leq$ $t_{f}(h)$. Pela invariância por multiplicação, segue que

$$
g \preceq h \Rightarrow g^{n} \preceq h^{n} \text {, para todo } n>0 \text {. }
$$

Por outro lado, temos para todo $n>0$ que

$$
f^{n t_{f, n}(g)} \preceq g^{n} \prec f^{n t_{f, n}(g)+1} .
$$

E então,

$$
f^{n t_{f, n}(g)} \preceq g^{n} \preceq h^{n}
$$

Mas por definição, também temos que

$$
f^{n t_{f, n}(h)} \preceq h^{n} \prec f^{n t_{f, n}(h)+1}
$$

Assim, concluímos que: $t_{f, n}(g) \leq t_{f, n}(h)$, e portanto,

$$
t_{f}(g)=\lim _{n \rightarrow+\infty} t_{f, n}(g) \leq \lim _{n \rightarrow+\infty} t_{f, n}(h)=t_{f}(h)
$$

Isso conclui a prova da afirmação.

Seja agora $g \in G, g \neq I d$. Temos então, ou $g \succ I d$, ou $g \prec I d$. Vamos supor o primeiro caso.

Como $g(x)>x$ para todo $x \in \mathbb{R}$, pelo Lema 2.2.14, segue que existe $n>0$, tal que: $g^{n}(0)>f(0)$. Então, $g^{n} \succ f$, e como o homomorfismo $g \mapsto t_{f}(g)$ é crescente, temos:

$$
1=t_{f}(f) \leq t_{f}\left(g^{n}\right)=t_{f}(g)+\ldots+t_{f}(g)=n t_{f}(g) .
$$

Assim, $t_{f}(g) \geq \frac{1}{n}$, e portanto: $t_{f}(g) \neq 0$.

Caso $g \prec I d$, multiplicando por $g^{-1}$, segue que $g^{-1} \succ I d$ e assim: $-t_{f}(g)=t_{f}\left(g^{-1}\right) \neq 0$.

Temos assim, que: $g \neq I d \Rightarrow t_{f}(g) \neq 0$, o que termina a prova.

Uma conclusão fantástica do homomorfismo injetivo $g \mapsto t_{f}(g)$ é o seguinte corolário:

Corolário 3.1.6 O grupo $G$ é abeliano. 
Prova. Para todo $g, h \in G$, temos:

$$
t_{f}(g \circ h)=t_{f}(g)+t_{f}(h)=t_{f}(h \circ g) .
$$

Em particular, $t_{f}(g \circ h)=t_{f}(h \circ g)$. Mas como a aplicação $g \mapsto t_{f}(g)$ é injetiva, temos: $g \circ h=h \circ g$.

Para terminar a prova do Teorema 3.1.1, resta construir a semiconjugação $h: \mathbb{R} \rightarrow \mathbb{R}$ entre o grupo $G$ e um grupo de translações de $\mathbb{R}$. Para isso, fixamos um ponto de $\mathbb{R}$, por exemplo o ponto 0 , e um elemento $f \in G \operatorname{com} f \succ I d$. Sejam $\mathcal{A}=G .0$ a órbita do ponto 0 e $\mathcal{B}=\left\{t_{f}(g) \mid g \in G\right\}$. Note que $\mathcal{B}$ é um subgrupo de $(\mathbb{R},+)$, e portanto o Lema 2.2.7 se aplica:

- Ou $\mathcal{B}$ é gerado por um único elemento (ou seja, $\mathcal{B}=\mathbb{Z} . b)$.

- Ou $\mathcal{B}$ é denso em $\mathbb{R}$.

Corolário 3.1.7 Se $\mathcal{B}$ é gerado por um único elemento então a ação $G$ é conjugada à um subgrupo de translações.

Prova. Como a aplicação $G \rightarrow \mathcal{B}$, definida por $g \mapsto t_{f}(g)$ é um isomorfismo de grupos, temos que $G$ também é gerado por um único elemento. Seja então, $g \in G$ um gerador.

Se $g=I d$, segue que o grupo é trivial, e como a identidade é uma translação, o grupo $G=\{I d\}$ é um subgrupo de translações.

Para o caso de $g$ não trivial, lembre que $G$ é livre de pontos fixos e portanto, $g$ também é um elemento livre de pontos fixos. Então, pelo Corolário 2.2.20 g pode ser conjugado à qualquer translação $t: \mathbb{R} \rightarrow \mathbb{R}$, por um homeomorfismo crescente $h: \mathbb{R} \rightarrow \mathbb{R}$, ou seja, para todo $x \in \mathbb{R}$ temos:

$$
h \circ g(x)=t \circ h(x)
$$

Por ser uma conjugação, segue que $g(x)=h^{-1} \circ t \circ h(x)$. Portanto, para todo $k \in \mathbb{Z}$ se verifica

$$
g^{k}(x)=\left(h \circ t \circ h^{-1}\right)^{k}(x)=h \circ t^{k} \circ h^{-1}(x)
$$

E portanto, para todo $x \in \mathbb{R}$ se verifica

$$
h \circ g^{k}(x)=t^{k} \circ h(x)
$$

Ou seja, o mesmo homeomorfismo $h$ conjuga todos os elementos gerados por $g$ em translações da forma $t^{k}$. Como o grupo $G$ é gerado por $g$, segue que $h$ conjuga o grupo $G$ a um subgrupo de translações. 
Corolário 3.1.8 Se $\mathcal{B}$ é denso em $\mathbb{R}$ então existe uma única $h: \mathbb{R} \rightarrow \mathbb{R}$ contínua, crescente e sobrejetiva que satisfaz para todo $g \in G$ e todo $x \in \mathbb{R}$,

$$
h \circ g(x)=h(x)+t_{f}(g)
$$

E o diagrama comuta:

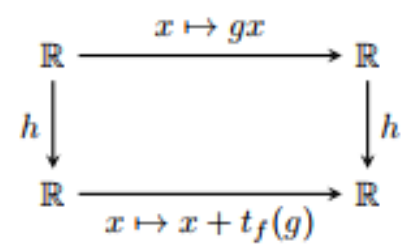

Prova. Lembre-se que $\mathcal{A}$ é a órbita do ponto 0. Defina a aplicação $h_{0}: \mathcal{A} \rightarrow \mathcal{B}$, por $g(0) \mapsto t_{f}(g)$. Note que ela é uma bijeção crescente, pois as aplicações $g(0) \mapsto g$ e $g \mapsto t_{f}(g)$ são bijeções crescentes (visto nos Lemas 3.1.2, 3.1.4 e 3.1.5), e como $\mathcal{B}$ é denso, pelo Lema 2.1.17, $h_{0}: \mathcal{A} \rightarrow \mathcal{B}$ possui uma única extensão para uma função continua, crescente e sobrejetiva $h: \mathbb{R} \rightarrow \mathbb{R}$. Agora, fixe qualquer $x \in \mathbb{R}$ e $g \in G$. Temos que mostrar que $h(x)+t_{f}(g)=h(g(x))$.

Seja $y \in \mathcal{A}$ com $y \leq x$. Pela definição do conjunto $\mathcal{A}$, existe $g_{y} \in G$ com $g_{y}(0)=y$ e por ser crescente, temos $g(y) \leq g(x)$. Assim, pela definição de $h_{0}$, temos que

$$
h_{0}(y)=h_{0}\left(g_{y}(0)\right)=t_{f}\left(g_{y}\right)
$$

Portanto, também temos

$$
h_{0}(g(y))=h_{0}\left(g \circ g_{y}(0)\right)=t_{f}\left(g \circ g_{y}\right)=t_{f}(g)+t_{f}\left(g_{y}\right)=t_{f}(g)+h_{0}(y) .
$$

Como $h$ é extensão contínua e crescente de $h_{0}$ e $\mathcal{A}$ é denso em $\mathbb{R}$, temos que

$$
h(x)+t_{f}(g)=\sup _{y \in \mathcal{A}, y \leq x}\left\{h_{0}(y)+t_{f}(g)\right\}=\sup _{y \in \mathcal{A}, y \leq x}\left\{h_{0}(g(y))\right\} .
$$

Mas como $g \in G$ é homeomorfismo crescente, segue que

$$
\sup _{y \in \mathcal{A}, y \leq x}\left\{h_{0}(g(y))\right\}=\sup _{z \in \mathcal{A}, z \leq g(x)}\left\{h_{0}(z)\right\}=h(g(x)) .
$$

Concluímos assim $h(x)+t_{f}(g)=h(g(x))$.

Com esses dois últimos resultados, concluímos a demonstração do Teorema 3.1.1.

Lema 3.1.9 (Unicidade da semi-conjugação) Sejam $G \subset \mathrm{Homeo}_{+}(\mathbb{R})$ um subgrupo livre de pontos fixos que não é gerado por um único elemento 
e $h^{\prime}: \mathbb{R} \rightarrow \mathbb{R}$ uma aplicação continua, crescente e sobrejetiva, que realiza uma semi-conjugação entre $G$ e um subgrupo de translações. Então $h^{\prime}=\phi \circ h$, onde $\phi: \mathbb{R} \rightarrow \mathbb{R}$ é da forma $t \mapsto$ a.t $+b$ e $h$ é a semi-conjugação construída no Teorema 3.1.1.

Prova. Por definição de $h^{\prime}$, para todo $g \in G$, existe um $t^{\prime}(g) \in \mathbb{R}$, tal que:

$$
h^{\prime}(g(x))=h^{\prime}(x)+t^{\prime}(g), \text { para todo } x \in \mathbb{R} .
$$

Além disso, a aplicação $g \mapsto t^{\prime}(g)$ é um morfismo de $(G, \circ)$ para um subgrupo de $(\mathbb{R},+)$.

Afirmação. Se $g \succ I d$, então $t^{\prime}(g)>0$.

Prova. Pelo Lema 2.2.14, para todo $x \in \mathbb{R}$ temos que $g^{n}(x) \rightarrow+\infty$, quando $n \rightarrow+\infty$. Como $h^{\prime}$ é crescente e sobrejetiva, isso implica que existe $x$ e $n>0$, com $h^{\prime}\left(g^{n}(x)\right)>h^{\prime}(x)$. Por outro lado, temos

$$
h^{\prime}\left(g^{n}(x)\right)=h^{\prime}(x)+t^{\prime}\left(g^{n}\right)=h^{\prime}(x)+n t^{\prime}(g) .
$$

Então $n t^{\prime}(g)>0$, e segue que $t^{\prime}(g)>0$.

Seja $f \in G$ com $f \succ I d$, o elemento fixado na construção de $h$ tal que $t(g)=t_{f}(g)$.

Afirmação. Para todo $g \in G$, temos $t^{\prime}(g)=t^{\prime}(f) \cdot t_{f}(g)$.

Prova. Ambas aplicações $g \mapsto t_{f}(g)$ e $g \mapsto t^{\prime}(g)$ são isomorfismos crescentes de $G$ sobre os subgrupos $t_{f}(G)$ e $t^{\prime}(G)$ de $(\mathbb{R},+)$. Obtemos assim um isomorfismo crescente definido por $t_{f}(g) \mapsto t^{\prime}(g)$ entre $t_{f}(G)$ e $t^{\prime}(G)$.

Agora, como ambos os grupos $t_{f}(G)$ e $t^{\prime}(G)$ não são gerados por um único elemento, segue que são densos em $\mathbb{R}$. Pelo Lema 2.1.17, a aplicação $t_{f}(g) \mapsto t^{\prime}(g)$ pode ser estendida de maneira única, como um homeomorfismo crescente de $\mathbb{R}$. Que é então, um automorfismo contínuo e crescente de $(\mathbb{R},+)$. Portanto, pelo Lema 2.2.32 é uma homotetia positiva. Assim, existe $\alpha>0$, tal que

$$
t^{\prime}(g)=\alpha \cdot t_{f}(g), \text { para todo } g \in G .
$$

Mas, como $t^{\prime}(f)=\alpha \cdot t_{f}(f)=\alpha .1=\alpha$. Segue a igualdade

$$
t^{\prime}(g)=t^{\prime}(f) \cdot t_{f}(g),
$$

concluindo a prova da afirmação. 
Afirmação. Existem $a, b \in \mathbb{R}$, tais que para todo $x \in \mathbb{R}$, temos

$$
h^{\prime}(x)=a \cdot h(x)+b .
$$

Prova. Note que, para todo $s>0$, a aplicação definida por

$$
h_{s}^{\prime}(x)=\frac{1}{s} h^{\prime}(x)
$$

define outra semi-conjugação entre o grupo $G$ e um subgrupo de translações. Com efeito:

$$
h_{s}^{\prime}(g(x))=\frac{1}{s} h^{\prime}(g(x))=\frac{1}{s}\left(h^{\prime}(x)+t^{\prime}(g)\right)=h_{s}^{\prime}(x)+\frac{t^{\prime}(g)}{s} .
$$

Fixamos então $s=t^{\prime}(f)$ e definirmos a seguinte aplicação:

$$
h_{1}(x):=h_{t^{\prime}(f)}^{\prime}(x)=\frac{1}{t^{\prime}(f)} h^{\prime}(x) .
$$

E então, pela afirmação anterior, $h_{1}$ é uma semi-conjugação, contínua, crescente e sobrejetiva, tal que para todo $x \in \mathbb{R}$,

$$
h_{1}(g(x))=h_{1}(x)+\frac{t^{\prime}(g)}{t^{\prime}(f)}=h_{1}(x)+t_{f}(g) .
$$

Denote por $\beta=h_{1}(0)$ e $h_{2}=h_{1}-\beta$. Então, $h_{2}$ é uma semi-conjugação tal que $h_{2}(0)=0$, e

$$
h_{2}(g(x))=h_{2}(x)+t_{f}(g) \text {, para todo } x \in \mathbb{R} \text { e todo } g \in G \text {. }
$$

Em particular, temos para todo $g \in G$

$$
h_{2}(g(0))=t_{f}(g)=h(g(0))
$$

Portanto, $h_{2}$ e $h$ coincidem em todo o conjunto $\mathcal{A}$. Como a sua imagem, $\mathcal{B}$ é densa, e a extensão como função contínua e crescente para todo $\mathbb{R}$ é única, segue que $h_{2}(x)=h(x)$, para todo $x \in \mathbb{R}$. Então,

$$
h=h_{2}=h_{1}-\beta=\frac{1}{t^{\prime}(f)} h^{\prime}-\beta
$$

e assim: $h^{\prime}=t^{\prime}(f) \cdot h+t^{\prime}(f) \beta$. Basta, por fim, definir $a:=t^{\prime}(f)$ e $b:=t^{\prime}(f) \beta$ e concluímos a prova da afirmação.

Concluímos assim a prova do lema. 


\section{2}

\section{Ações com um ponto fixo global}

Um ponto fixo global divide a reta em duas componentes invariantes, e nesse caso como o único ponto fixo da ação é o global, a ação será livre de pontos fixos em cada componente. Aplicando o Teorema de Hölder em uma das componentes, seguirá diretamente que o grupo é abeliano!

Começamos apresentando um exemplo clássico de ação com um ponto fixo global. Veremos que a menos de semi-conjugação, este é o único exemplo, de ações de grupos com um único ponto fixo global, possível.

Definição 3.2.1 Seja $\left\{a_{i}\right\}_{i \in \Omega} \subset \mathbb{R}$ uma família de números reais. Dizemos que a família $\left\{a_{i}\right\}_{i \in \Omega}$ é racionalmente independente, se satisfaz para quaisquer $n$-ulpa $i_{1}, \ldots, i_{n} \in \Omega$,

$$
q_{1} \cdot a_{i_{1}}+\ldots+q_{n} \cdot a_{i_{n}}=0 \operatorname{com} q_{1}, \ldots, q_{n} \in \mathbb{Q} \Leftrightarrow q_{1}=\ldots=q_{n}=0 .
$$

\section{Exemplo 3.2.2 (Ação de grupo com um único ponto fixo global) \\ Considere duas famílias de números reais $\left\{a_{i}\right\}_{i \in \Omega}$ e $\left\{b_{i}\right\}_{i \in \Omega}$, racionalmente independentes, e considere o grupo gerado $F:=\left\langle f_{i}\right\rangle$, onde $f_{i}$ coincide com a homotetia de razão $a_{i}$ em $[0,+\infty)$ e de razão $b_{i}$ em $(-\infty, 0]$. Então 0 é o único ponto fixo da ação de $F$ sobre $\mathbb{R}$ e é um ponto fixo global.}

Teorema 3.2.3 Seja $G \subset$ Homeo $_{+}(\mathbb{R})$ subgrupo, com no máximo um ponto fixo. Se existe ponto fixo global então $G$ é abeliano e semi-conjugado a um dos grupos descritos no Exemplo 3.2.2.

Prova. Seja $x_{0}$ o ponto fixo global. Então as componentes $\left(-\infty, x_{0}\right)$ e $\left(x_{0},+\infty\right)$ são invariantes pela ação de $G$. Além disso, $G$ age em cada componente livre de pontos fixos, pois $x_{0}$ deve ser o único ponto fixo de qualquer elemento nãotrivial do grupo $G$. Porém, cada uma das componentes é homeomorfa a $\mathbb{R}$. Fixe um homeomorfismo $f:\left(x_{0},+\infty\right) \rightarrow \mathbb{R}$, note que $f$ induz uma conjugação da ação de $G$ sobre $\mathbb{R}$ que também será livre de pontos fixos. Portanto, aplicando o Teorema de Hölder, temos que $G$ é abeliano e, além disso, existe $h: \mathbb{R} \rightarrow \mathbb{R}$ contínua, crescente e sobrejetiva que realiza uma semi-conjugação com um subgrupo de translações.

Por fim, $\mathbb{R}$ é homeomorfo à componente $(0,+\infty)$ pelo homeomorfismo $x \mapsto \exp (x)$. Note que a exponencial exp induz uma conjugação que leva um subgrupo de translações para um subgrupo de homotetias. Temos assim os seguintes diagramas comutativos 


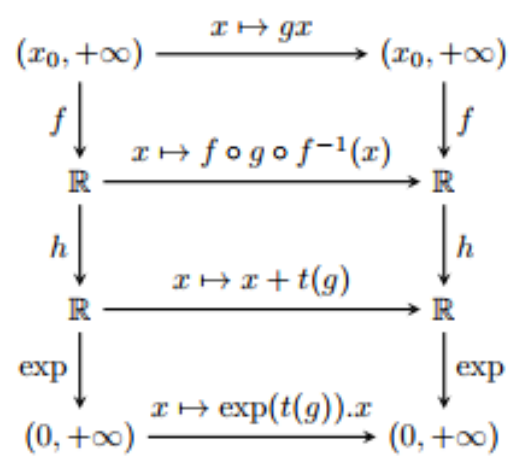

A aplicação exp ohof é contínua, crescente e sobrejetiva, e ela define uma semi-conjugação entre a restrição da ação de $G$ sobre a componente $\left(x_{0},+\infty\right)$ e um subgrupo das homotetias de $(0,+\infty)$.

De forma análoga, temos uma semi-conjugação entre a restrição da ação de $G$ sobre a componente $\left(-\infty, x_{0}\right)$ e um subgrupo das homotetias de $(-\infty, 0)$. Juntando as duas componentes, temos uma semi-conjugação da ação de $G$ sobre um grupo de homotetias à direita e à esquerda do ponto 0 .

Porém, como a ação é livre de pontos fixos em $(-\infty, 0) \cup(0,+\infty)$, o único elemento de $G$ que pode ser associado com a homotetia trivial (identidade na componente) é o elemento $I d \in G$. Por conta disso, as homotetias devem ter geradores racionalmente independentes. Assim, concluímos que $G$ é semiconjugado à um grupo como o descrito no Exemplo 3.2.2.

\section{3}

\section{O Teorema de Solodov}

Vamos considerar agora, ações com no máximo um ponto fixo, porém sem pontos fixos globais. Nesse caso temos uma relação de ordem, que apesar de não ser arquimediana, possui propriedades similares, e com ela vamos definir um morfismo de grupos similar ao feito no Teorema de Hölder, porém dessa vez, por não ser arquimediana, o morfismo não será injetivo.

Observaremos que o núcleo do morfismo é formado exatamente pelos elementos livres de pontos fixos do grupo, assim podemos aplicar o Teorema de Hölder e concluir que o núcleo é abeliano, além de ter uma relação de ordem arquimediana e uma aplicação $\phi: \mathbb{R} \rightarrow \mathbb{R}$ semi-conjugando a ação do núcleo com a ação de um grupo de translações.

Por fim, de forma inesperada, vamos perceber que para cada elemento do grupo $g \in G$, a aplicação $\phi \circ g: \mathbb{R} \rightarrow \mathbb{R}$ também semi-conjuga o núcleo com as translações. Portanto, usando a unicidade das semi-conjugações de Hölder (Lema 3.1.9), concluímos que para cada $g \in G$ existem constantes $a_{g}$ e $b_{g}$, tais 
que

$$
\phi \circ g=a_{g} \cdot \phi+b_{g}
$$

concluindo assim uma semi-conjugação da ação do grupo $G$ com a ação de um grupo de aplicações afins.

Começaremos então, enunciando o principal resultado desse capítulo, o Teorema de Solodov.

Teorema 3.3.1 (Teorema de Solodov) Seja $G \subset$ Homeo $_{+}(\mathbb{R})$ um grupo agindo com no máximo um ponto fixo. Então um dos itens abaixo ocorre:

- G possui um ponto fixo global. E nesse caso, G é abeliano.

- Existe um aplicação crescente e continua $\phi: \mathbb{R} \rightarrow \mathbb{R}$ e duas aplicações $a, b: G \rightarrow \mathbb{R}$, tais que para todo $g \in G$ e todo $x \in \mathbb{R}$ se verifica

$$
\phi \circ g(x)=a(g) \cdot \phi(x)+b(g)
$$

Ou seja, o diagrama comuta:

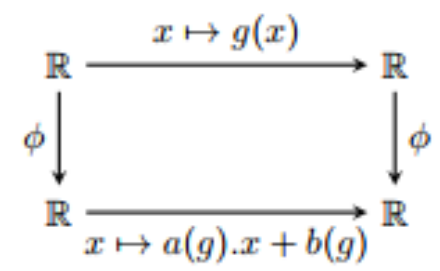

Prova. O primeiro caso está claro, pelo Teorema 3.2.3. Agora, vamos assumir $G$ não tem ponto fixo global.

Lema 3.3.2 Suponha $g \in G$ tal que $g \preceq I d$ (i.e., $g(x) \leq x$ para todo $x \in \mathbb{R}$ ), ou $g \succeq I d$ (i.e., $g(x) \geq x$ para todo $x \in \mathbb{R}$ ). Então $g$ é livre de pontos fixos.

Prova. Suponha por absurdo, que $g$ possui um ponto fixo $x_{0}$ e $g \preceq I d$. Segue, pelo Lema 2.2.14, que

$$
\begin{gathered}
\lim _{n \rightarrow+\infty} g^{n}(x)=-\infty, \text { para todo } x<x_{0}, \\
\lim _{n \rightarrow+\infty} g^{n}(x)=x_{0}, \text { para todo } x>x_{0} .
\end{gathered}
$$

Como o ponto $x_{0}$ não é um ponto fixo global, existe $h \in G$ com $h\left(x_{0}\right) \neq x_{0}$. Trocando $h$ por $h^{-1}$, se necessário, podemos supor que $h\left(x_{0}\right)>x_{0}$.

Vamos definir a função conjugada $f:=h \circ g \circ h^{-1}$. Note que seu ponto fixo é $x_{1}=h\left(x_{0}\right)>x_{0}$.

Afirmação. Para todo $x \in \mathbb{R}$ temos que $f(x) \leq x$. 
Prova. Por hipótese, temos $g(x) \leq x$ para todo $x \in \mathbb{R}$. Em particular: $g\left(h^{-1}(x)\right) \leq h^{-1}(x)$. E como $h$ é crescente, segue que

$$
h \circ g\left(h^{-1}(x)\right) \leq h \circ h^{-1}(x)=x .
$$

Concluímos assim que $f(x) \leq x$.

Afirmação. Existem $q \in G$ e $y \in \mathbb{R}$ tais que:

$$
q(y)<y<x_{0}<q\left(x_{0}\right)<q\left(x_{1}\right)<x_{1}
$$

Prova. Verifica-se $\lim _{n \rightarrow+\infty} g^{n}(x)=-\infty$, para todo $x<x_{0}$. Fixando $y<x_{0}$, existe $n_{0} \in \mathbb{N}$, tal que $g^{n_{0}}(y)<f(y)$. Além disso, para todo $n \in \mathbb{N}$, temos $g^{n}\left(x_{0}\right)=x_{0}$. E, como $f(x)<x$, para todo $x \neq x_{1}$, temos:

$$
g^{n}\left(x_{0}\right)=x_{0}>f\left(x_{0}\right)
$$

Por fim, para todo $n \in \mathbb{N}$, temos $g^{n} \preceq I d$, pois $g \preceq I d$ (Lema 2.2.14 aplicado em $g^{-1}$ ), portanto $g^{n}(x)<x$ para todo $x \neq x_{0}$. Assim, temos:

$$
g^{n}\left(x_{1}\right)<x_{1}=f\left(x_{1}\right)
$$

Defina agora, $q:=f^{-1} \circ g^{n_{0}}$. Portanto, temos as seguintes desigualdades:

$$
\begin{gathered}
g^{n_{0}}(y)<f(y) \Rightarrow f^{-1} \circ g^{n_{0}}(y)<y \Rightarrow q(y)<y, \\
g^{n_{0}}\left(x_{0}\right)>f\left(x_{0}\right) \Rightarrow f^{-1} \circ g^{n_{0}}\left(x_{0}\right)>x_{0} \Rightarrow q\left(x_{0}\right)>x_{0}, \\
g^{n_{0}}\left(x_{1}\right)<f\left(x_{1}\right) \Rightarrow f^{-1} \circ g^{n_{0}}\left(x_{1}\right)<x_{1} \Rightarrow q\left(x_{1}\right)<x_{1} .
\end{gathered}
$$

Como $q\left(x_{0}\right)<q\left(x_{1}\right)$, pois $q$ é estritamente crescente e $x_{0}<x_{1}$, provamos a afirmação.

Agora, note que $y<x_{0}<x_{1}$. Assim, o sinal de $q(x)-x$ troca pelo menos duas vezes entre $y$ e $x_{1}$. E portanto, pela Observação 2.1.11, $q$ possui pelo menos dois pontos fixos. Mas como $q \in G$, temos que $q$ é necessariamente um elemento trivial, ou seja, $q=I d$. O que é uma contradição com a afirmação anterior.

Por fim, caso $g \succeq I d$, temos que $g^{-1} \preceq I d$, e $g^{-1}$ livre de pontos fixos, implica em $g$ livre de pontos fixos. Assim concluímos a prova do lema.

Defina a seguinte relação em $G$ : dados $f, g \in G$, dizemos que

$$
f \triangleleft g \text { se existe } C \in \mathbb{R} \text { tal que: } f(x)<g(x) \text { para todo } x \geq C \text {. }
$$


De forma similar, escrevemos $g \triangleright f$ se $f \triangleleft g$.

Lema 3.3.3 A relação $\triangleleft$ é uma relação de ordem total sobre G. Além disso, ela é invariante por multiplicação à direita e à esquerda pelos elementos de $G$.

Prova. A demonstração será consequência das seguintes afirmações.

Afirmação. $\triangleleft$ é uma ordem total estrita.

Prova. Basta verificar cada um dos itens abaixo:

- Transitividade: Se $f \triangleleft g$ e $g \triangleleft h$, então, existem $C_{1}$ e $C_{2}$ tais que: para todo $x \geq \max \left\{C_{1}, C_{2}\right\}$, temos: $f(x)<g(x)<h(x)$. E portanto, $f \triangleleft h$.

- Assimetria: Se $f \triangleleft g$, então, exite $C \in \mathbb{R}$, tal que: para todo $x \geq C$, temos: $f(x)<g(x)$. E portanto, é claro que $g \Varangle f$.

- Irreflexividade: Pela definição de $\triangleleft$, está claro que $f \Varangle f$.

- Totalidade: Basta notar que os gráficos de dois elementos distintos $f, g \in G$ se cortam no máximo uma vez, caso contrário $g^{-1} \circ f$ teria mais do que um ponto fixo. Portanto, sempre existe $C$, tal que: ou $f(x)>g(x)$ para todo $x \geq C$, ou $f(x)<g(x)$ para todo $x \geq C$.

Assim, a prova da afirmação está completa.

Afirmação. Para todo $f, g, h \in G$, temos:

- $f \triangleleft g \Rightarrow h \circ f \triangleleft h \circ g$ (invariante por multiplicação à esquerda),

- $f \triangleleft g \Rightarrow f \circ h \triangleleft g \circ h$ (invariante por multiplicação à direita).

Prova. Se $f \triangleleft g$ então existe $C \in \mathbb{R}$ tal que $f(x)<g(x)$, para todo $x \geq C$. Como $h \in G$ é crescente, temos que: $h(f(x))<h(g(x))$, para todo $x \geq C$. E assim, $h \circ f \triangleleft h \circ g$.

Por outro lado, como $h: \mathbb{R} \rightarrow \mathbb{R}$ é homeomorfismo: se $f(x)<g(x)$, para todo $x \geq C$, então temos que: $f(h(y))<g(h(y))$, onde $y=h^{-1}(x)$. Ou seja, $f(h(y))<g(h(y))$ para todo $y \geq h^{-1}(C)$. E então, $f \circ h \triangleleft g \circ h$.

Assim concluímos a demonstração do lema.

Lema 3.3.4 Seja $f \in G$ com um ponto fixo e $f \triangleright I d$. Então, para todo $g \in G$, existe $n \in \mathbb{N}$ tal que $f^{n} \triangleright g$.

Prova. Seja $x_{0}$ o ponto fixo de $f$. Como $f \triangleright I d$, pelo Lema 3.3.2, temos que $f(x)<x$, para todo $x<x_{0}$ e $f(x)>x$, para todo $x>x_{0}$. Portanto, pelo Lema 2.2.14, temos:

$$
\lim _{n \rightarrow+\infty} f^{n}(x)=-\infty, \text { para todo } x<x_{0},
$$




$$
\lim _{n \rightarrow+\infty} f^{n}(x)=+\infty \text {, para todo } x>x_{0} .
$$

Fixe então, $x_{1}<x_{0}$ e $x_{2}>x_{0}$. Em particular, dado qualquer $g \in G$ existe $n \in \mathbb{N}$ suficientemente grande, com $f^{n}\left(x_{1}\right)<g\left(x_{1}\right)$ e $f^{n}\left(x_{2}\right)>g\left(x_{2}\right)$. Portanto, os gráficos $f^{n}$ e $g$ tem sua única interseção entre $x_{1}$ e $x_{2}$. E assim, $f^{n}(x)>g(x)$, para todo $x \geq x_{2}$. Implicando em: $f^{n} \triangleright g$.

Vamos proceder de forma similar ao número de translção durante a demonstração do Teorema de Hölder. Fixando $f \in G$ com ponto fixo $x_{0}$ e tal que $f \triangleright I d$, para cada $g \in G$ e cada $n \in \mathbb{N}$ definiremos o seguinte número racional

$$
\rho_{f, n}(g):=\frac{p}{n}, \text { tal que: } f^{p} \unlhd g^{n} \triangleleft f^{p+1}
$$

que está bem definido, graças ao Lema 3.3.4 que fornece uma espécie de relação de ordem arquimediana parcial.

Os Lemas 3.3.5 e 3.3.6 têm demonstrações idênticas às feitas no Teorema de Hölder (veja os Lemas 3.1.3 e 3.1.4), e portanto serão aqui omitidas.

Lema 3.3.5 A sequência $\left\{\rho_{f, n}(g)\right\}_{n \in \mathbb{N}}$ converge quando $n \rightarrow \infty$. E denotaremos o limite como $\rho_{f}(g)$.

Lema 3.3.6 A aplicação $(G, \circ) \rightarrow(\mathbb{R},+)$, definida por $g \mapsto \rho_{f}(g)$, é um homomorfismo crescente de grupos.

Vamos agora, concluir que $\operatorname{ker}\left(\rho_{f}\right)$ é formado pelos elementos livres de pontos fixos:

Lema 3.3.7 Se $h \in \operatorname{ker}\left(\rho_{f}\right)$ então h é livre de pontos fixos.

Prova. Se $h$ possui um ponto fixo e $h \triangleright I d$, vimos no Lema 3.3.4 que existe $n \in \mathbb{N}$, tal que $h^{n} \triangleright f$. Então,

$$
1=\rho_{f}(f) \leq \rho_{f}\left(h^{n}\right)=n . \rho_{f}(h) .
$$

Em particular, $h \notin \operatorname{ker}\left(\rho_{f}\right)$. E caso $h \not I d$, temos: $h^{-1} \triangleright I d$. E assim, $h^{-1} \notin \operatorname{ker}\left(\rho_{f}\right)$, o que implica em $h \notin \operatorname{ker}\left(\rho_{f}\right)$.

Lema 3.3.8 Se $h \in G$ é livre pontos fixos então $h \in \operatorname{ker}\left(\rho_{f}\right)$.

Prova. Seja $h \in G$ livre de pontos fixos e suponha $h(x)>x$, para todo $x \in \mathbb{R}$. Vamos mostrar que $\rho_{f}(h) \leq 1$. Supomos então, por absurdo, que $\rho_{f}(h)>1$.

Afirmação. Para todo $K \in G$ e para todo $x \in \mathbb{R}$, temos:

$$
K \circ h \circ K^{-1}(x) \geq f(x) .
$$


Prova. Como $h(x)>x$ para todo $x \in \mathbb{R}$, segue que

$$
h\left(K^{-1}(x)\right)>K^{-1}(x) \Rightarrow K \circ h \circ K^{-1}(x)>x .
$$

Assim, como $f \triangleright I d$ e $f$ possui ponto fixo $x_{0}$, segue que para todo $x \leq x_{0}$

$$
f(x) \leq x<K \circ h \circ K^{-1}(x)
$$

Além disso, como $\rho_{f}$ é morfismo, temos:

$$
\rho_{f}\left(K \circ h \circ K^{-1}\right)=\rho_{f}(K)+\rho_{f}(h)-\rho_{f}(K)=\rho_{f}(h)>1 .
$$

Assim, existe $C \in \mathbb{R}$, tal que $K \circ h \circ K^{-1}(x)>f(x)$, para todo $x \geq C$.

Agora estamos prontos para provar a afirmação. Suponha então, por absurdo, que existe $y \in \mathbb{R}$ com $K \circ h \circ K^{-1}(y)<f(y)$. Necessariamente, $x_{0}<y<C$. Defina $q:=f^{-1} \circ K \circ h \circ K^{-1} \in G$, segue que:

$$
K \circ h \circ K^{-1}\left(x_{0}\right)>x_{0}=f\left(x_{0}\right) \Rightarrow f^{-1} \circ K \circ h \circ K^{-1}\left(x_{0}\right)>x_{0} \Rightarrow q\left(x_{0}\right)>x_{0},
$$

$$
K \circ h \circ K^{-1}(y)<f(y) \Rightarrow f^{-1} \circ K \circ h \circ K^{-1}(y)<y \Rightarrow q(y)<y,
$$

$K \circ h \circ K^{-1}(C)>f(C) \Rightarrow f^{-1} \circ K \circ h \circ K^{-1}(C)>C \Rightarrow q(C)>C$.

E portanto, $q(x)-x$ troca de sinal pelo menos duas vezes, e assim, $q \in G$ tem pelo menos dois pontos fixos. Concluímos assim, $q=I d$. O que é uma contradição, pois $q\left(x_{0}\right) \neq x_{0}$.

Seja agora $k \in G$ com um ponto fixo $x_{k}>x_{0}$ e $k \triangleright I d$. Por exemplo, podemos escolher $k=h \circ f \circ h^{-1}$ e $x_{k}=h\left(x_{0}\right)>x_{0}$.

Afirmação. Existem $n>0$ e $z \in \mathbb{R}$ tais que $k^{-n} \circ h \circ k^{n}(z)<f(z)$.

Prova. Escolha $w>x_{k}$ e $y=h(w)>w$. E para todo $n>0$, defina $w_{n}=k^{-n}(w)$ e $y_{n}=k^{-n}(y)$. Note que para todo $n>0$ :

$$
k^{-n} \circ h \circ k^{n}\left(w_{n}\right)=y_{n}>w_{n}>x_{k} .
$$

Sabemos que para todo $x>x_{k}, k^{-n}(x)$ converge para $x_{k}$. Assim, como $y>w>x_{k}$, temos que $w_{n}$ e $y_{n}$ também convergem para $x_{k}$. Por fim, como $x_{k}>x_{0}$, temos: $f\left(x_{k}\right)>x_{k}$ e portanto, podemos escolher $n_{0} \in \mathbb{N}$, tal que: $y_{n_{0}}<f\left(x_{k}\right)$. Em particular, temos:

$$
k^{-n_{0}} \circ h \circ k^{n_{0}}\left(w_{n_{0}}\right)=y_{n_{0}}<f\left(x_{k}\right)<f\left(w_{n_{0}}\right) .
$$


Escolhendo $n=n_{0}$ e $z=w_{n_{0}}$, a prova da afirmação está completa.

Agora, tomando $K=k^{-n_{0}} \in G$, temos uma contradição entre as duas afirmações anteriores. Portanto, para toda $h \in G$ livre de pontos fixos com $h \succ I d$, temos $\rho_{f}(h) \leq 1$. Em particular, para todo $n>0, h^{n} \in G$ é livre de pontos fixos e $h^{n} \succ I d$. Assim, temos:

$$
\rho_{f}\left(h^{n}\right) \leq 1 \Rightarrow \rho_{f}(h) \leq \frac{1}{n} .
$$

Como $h \succ I d$, temos também $\rho_{f}(h) \geq \rho_{f}(I d)=0$. Portanto, $h \in \operatorname{ker}\left(\rho_{f}\right)$.

Caso $h \prec I d$, temos $h^{-1} \succ I d$ e sem pontos fixos. Portanto: $\rho_{f}\left(h^{-1}\right)=0$. E assim

$$
\rho_{f}(h)=-\rho_{f}\left(h^{-1}\right)=0 .
$$

Concluímos portanto, a demonstração do Lema 3.3.8.

Agora, note que como não existem pontos fixos globais e $G$ tem no máximo um ponto fixo, pelo Lema 2.2.28 temos $G$ não-abeliano. Portanto, existem $h, g \in G$ que não comutam, ou seja, $q=g \circ h \circ g^{-1} \circ h^{-1} \neq I d$. Porém, temos que

$$
\rho_{f}(q)=\rho_{f}\left(g \circ h \circ g^{-1} \circ h^{-1}\right)=\rho_{f}(g)+\rho_{f}(h)-\rho_{f}(g)-\rho_{f}(h)=0 .
$$

Ou seja, o elemento $q$ pertence ao $\operatorname{ker}\left(\rho_{f}\right)$. E então, temos que $\operatorname{ker}\left(\rho_{f}\right) \subset G$ é um subgrupo não trivial. Além disso, vimos no Lema 3.3.8 e Lema 3.3.7 que

$$
h \in \operatorname{ker}\left(\rho_{f}\right) \Leftrightarrow h \text { é livre de pontos fixos. }
$$

Portanto, $\operatorname{ker}\left(\rho_{f}\right) \subset \mathrm{Homeo}_{+}(\mathbb{R})$ age sobre $\mathbb{R}$ livre de pontos fixos. E, pelo Teorema de Hölder, $\operatorname{ker}\left(\rho_{f}\right)$ é naturalmente munido de uma ordem total $\prec$, invariante por multiplicação à direita e à esquerda, definida por:

$$
h \prec g \Leftrightarrow h(x)<g(x) \text {, para todo } x \in \mathbb{R}, \operatorname{com} h, g \in \operatorname{ker}\left(\rho_{f}\right) \text {. }
$$

Lema 3.3.9 O grupo $\operatorname{ker}\left(\rho_{f}\right)$ não é gerado por um único elemento.

Prova. Se $T$ é um elemento de $\operatorname{ker}\left(\rho_{f}\right)$ com $T \succ I d$, como $f$ é não-trivial e possui um ponto fixo $x_{0}$, temos que $T$ e $f$ não comutam. Em efeito: $T\left(x_{0}\right)>x_{0}$ e $f(x)>x$ para todo $x>x_{0}$, e assim

$$
f\left(T\left(x_{0}\right)\right)>T\left(x_{0}\right)=T\left(f\left(x_{0}\right)\right) .
$$


Note que $\rho_{f}\left(f \circ T \circ f^{-1}\right)=\rho_{f}(T)=0$. Portanto, $f \circ T \circ f^{-1} \in \operatorname{ker}\left(\rho_{f}\right)$. Assim, $f \circ T \circ f^{-1}$ e $T$ são dois elementos diferentes de $\operatorname{ker}\left(\rho_{f}\right)$. Segue que:

$$
T \prec f \circ T \circ f^{-1} \quad \text { ou } \quad T \succ f \circ T \circ f^{-1} .
$$

Vamos supor $T \succ f \circ T \circ f^{-1}$.

Afirmação. Para todo $n \in \mathbb{N}$,

$$
f^{n} \circ T \circ f^{-n} \succ f^{n+1} \circ T \circ f^{-(n+1)} .
$$

Prova. Vamos demonstrar a afirmação por indução em $n$. Por hipótese, temos $T \succ f \circ T \circ f^{-1}$. Pela definição de $\prec$, temos para todo $x \in \mathbb{R}$

$$
T(x)>f \circ T \circ f^{-1}(x) .
$$

E assim, como a ordem é invariante por multiplicação à direita e à esquerda,

$$
f^{n} \circ T \circ f^{-n}(x)>f^{n} \circ\left(f \circ T \circ f^{-1}\right) \circ f^{-n}(x)=f^{(n+1)} \circ T \circ f^{-(n+1)}(x) .
$$

Ou seja, $f^{n} \circ T \circ f^{-n} \succ f^{n+1} \circ T \circ f^{-(n+1)}$.

Portanto, os homeomorfismos $\left(f^{n} \circ T \circ f^{-n}\right)$ formam uma sequência decrescente em $\operatorname{ker}\left(\rho_{f}\right)$, cotada inferiormente pela $I d$. Com efeito, para todo $n \in \mathbb{N}$ e para todo $x \in \mathbb{R}$ temos

$$
T(x)>x \Rightarrow T\left(f^{-n}(x)\right)>f^{-n}(x) \Rightarrow f^{n} \circ T \circ f^{-n}(x)>f^{n}\left(f^{-n}(x)\right)=x .
$$

Assim, é impossível que $\operatorname{ker}\left(\rho_{f}\right)$ seja isomorfo ao grupo ordenado $(\mathbb{Z},+,<)$, e assim não é gerado por um único elemento.

Caso $T \prec f \circ T \circ f^{-1}$, então $f^{-1} \circ T \circ f \prec T$. Trocando $f$ e $f^{-1}$ de papel, o mesmo resultado segue.

Lema 3.3.10 Dado $k \in G$ considere a aplicação $\psi_{k}: \operatorname{ker}\left(\rho_{f}\right) \rightarrow \operatorname{ker}\left(\rho_{f}\right)$, definida por: $g \mapsto k \circ g \circ k^{-1}$. A aplicação $\psi_{k}$ é um morfismo de grupos, crescente com relação à ordem $\prec$. Além disso, $\psi_{k_{1} \circ k_{2}}=\psi_{k_{1}} \circ \psi_{k_{2}}$.

Prova. Note que, para qualquer $g_{1}, g_{2} \in \operatorname{ker}\left(\rho_{f}\right)$, temos:

$$
\psi_{k}\left(g_{1}\right) \circ \psi_{k}\left(g_{2}\right)=k \circ g_{1} \circ k^{-1} \circ k \circ g_{2} \circ k^{-1}=k \circ g_{1} \circ g_{2} \circ k^{-1} \text {. }
$$

Logo, $\psi_{k}$ é morfismo de grupo. 
Se $g_{1} \prec g_{2}$, então para todo $x \in \mathbb{R}: g_{1}(x)<g_{2}(x)$. Assim, como $k$ é homeomorfismo crescente, temos:

$$
g_{1}\left(k^{-1}(y)\right)<g_{2}\left(k^{-1}(y)\right), \text { para todo } y \in \mathbb{R}
$$

E portanto, $k\left(g_{1}\left(k^{-1}(y)\right)\right)<k\left(g_{2}\left(k^{-1}(y)\right)\right)$, ou seja, $\psi_{k}\left(g_{1}\right)(y)<\psi_{k}\left(g_{2}\right)(y)$ para todo $y \in \mathbb{R}$. Concluímos então que $\psi_{k}\left(g_{1}\right) \prec \psi_{k}\left(g_{2}\right)$.

Por fim, note que para todo $k_{1}, k_{2} \in G$ e todo $g \in \operatorname{ker}\left(\rho_{f}\right)$ temos

$\psi_{k_{1} \circ k_{2}}(g)=\left(k_{1} \circ k_{2}\right) \circ g \circ\left(k_{1} \circ k_{2}\right)^{-1}=k_{1} \circ\left(k_{2} \circ g \circ k_{2}^{-1}\right) \circ k_{1}^{-1}=\psi_{k_{1}}\left(\psi_{k_{2}}(g)\right)$,

o que conclui a prova do lema.

Lembrando que como $\operatorname{ker}\left(\rho_{f}\right)$ age livre de pontos fixos sobre $\mathbb{R}$, pelo Teorema de Hölder, para cada $h \in \operatorname{ker}\left(\rho_{f}\right), h \succ I d$, existe $\phi: \mathbb{R} \rightarrow \mathbb{R}$ contínua, crescente e sobrejetiva, tal que a aplicação $\phi$ é uma semi-conjugação com um grupo de translações.

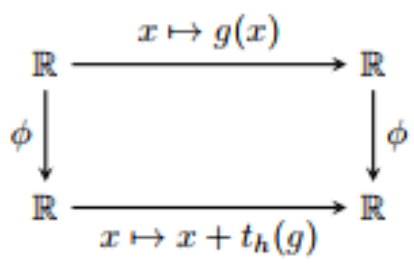

Vamos então fixar um elemento $h \in \operatorname{ker}\left(\rho_{f}\right), \operatorname{com} h \succ I d$.

Corolário 3.3.11 Para todo $k \in G$, existe $\alpha(k) \in \mathbb{R}$, estritamente positivo, tal que para todo $g \in \operatorname{ker}\left(\rho_{f}\right)$, temos:

$$
t_{h}\left(\psi_{k}(g)\right)=\alpha(k) \cdot t_{h}(g)
$$

Além disso, a aplicação $k \mapsto \alpha(k)$ é um homomorfismo de $(G, \circ)$ para $\left(\mathbb{R}_{+}^{*}, \times\right)$.

Prova. Denote por $T:=t_{h}\left(\operatorname{ker}\left(\rho_{f}\right)\right) \subset \mathbb{R}$. Pelo Lema 3.3.9, temos que $\operatorname{ker}\left(\rho_{f}\right)$ não é gerado por um único elemento, e portanto o mesmo vale para $T=t_{h}\left(\operatorname{ker}\left(\rho_{f}\right)\right)$, já que a aplicação $g \mapsto t_{h}(g)$ é um isomomorfismo (visto em 3.1.5). Agora, como $T \subset \mathbb{R}$ é subgrupo que não pode ser gerado por um único elemento, segue pelo Lema 2.2.7, que $T$ é um subgrupo denso de $\mathbb{R}$.

Como $t_{h}: \operatorname{ker}\left(\rho_{f}\right) \rightarrow T$ é um isomorfismo crescente (Lema 3.1.5), deduzimos que a aplicação $t_{h}(g) \mapsto t_{h}\left(\psi_{k}(g)\right)$ é um automorfismo crescente de $T$. 
Essa aplicação se estende para um automorfismo contínuo e crescente de $\mathbb{R}$. E pelo Lema 2.2.32, segue que é uma homotetia de razão $\alpha(k)>0$. Ou seja,

$$
t_{h}\left(\psi_{k}(g)\right)=\alpha(k) \cdot t_{h}(g)
$$

Além disso, usando o Lema 3.3.10, se verifica para todo $k_{1}, k_{2} \in G$ e todo $g \in \operatorname{ker}\left(\rho_{f}\right)$ que

$$
\begin{gathered}
\alpha\left(k_{1} \circ k_{2}\right) \cdot t_{h}(g)=t_{h}\left(\psi_{k_{1} \circ k_{2}}(g)\right)=t_{h}\left(\psi_{k_{1}}\left(\psi_{k_{2}}(g)\right)\right)= \\
=\alpha\left(k_{1}\right) \cdot t_{h}\left(\psi_{k_{2}}(g)\right)=\alpha\left(k_{1}\right) \cdot \alpha\left(k_{2}\right) \cdot t_{h}(g),
\end{gathered}
$$

provando que a aplicação é um morfismo de grupos e concluindo a prova do lema.

Lema 3.3.12 Para todo $k \triangleright I d$ com um ponto fixo, temos que $\alpha(k)>1$. Além disso, a aplicação $k \mapsto \alpha(k)$ é um morfismo crescente de $(G, \circ, \unlhd)$ sobre $((0,+\infty), \times, \leq)$.

Prova. Seja $k \triangleright I d$ com um ponto fixo $x_{k}$. Lembre-se que $h \in \operatorname{ker}\left(\rho_{f}\right)$ foi escolhido tal que $h \succ I d$ e $t_{h}(h)=1$.

Para todo $n>0$, temos $k^{-n} \circ h \circ k^{n}=\psi_{k^{-n}}(h)$. Por definição,

$$
t_{h}\left(k^{-n} \circ h \circ k^{n}\right)=t_{h}\left(\psi_{k^{-n}}(h)\right)=\alpha\left(k^{-n}\right) \cdot t_{h}(h)=\alpha\left(k^{-n}\right)=\alpha(k)^{-n} .
$$

Agora, escolha $w>x_{k}$ e $y=h(w)>w$, e para todo $n>0$, defina $w_{n}:=k^{-n}(w)$ e $y_{n}:=k^{-n}(y)$. Note que

$$
k^{-n} \circ h \circ k^{n}\left(w_{n}\right)=y_{n}>w_{n}>x_{k} .
$$

Sabemos que para todo $x>x_{k}, k^{-n}(x)$ converge para $x_{k}$. Assim, como $y>w>x_{k}$ temos: $w_{n}$ e $y_{n}$ convergem para $x_{k}$.

Por fim, como $h \succ I d$, temos $h\left(x_{k}\right)>x_{k}$ e portanto, podemos escolher $n_{0} \in \mathbb{N}$, tal que $y_{n_{0}}<h\left(x_{k}\right)$. Em particular, temos:

$$
k^{-n_{0}} \circ h \circ k^{n_{0}}\left(w_{n_{0}}\right)=y_{n_{0}}<h\left(x_{k}\right)<h\left(w_{n_{0}}\right) .
$$

Deduzimos assim, $k^{-n_{0}} \circ h \circ k^{n_{0}}\left(w_{n_{0}}\right) \prec h$. E então:

$$
1>t_{h}\left(k^{-n_{0}} \circ h \circ k^{n_{0}}\left(w_{n_{0}}\right)\right)=\alpha(k)^{-n} .
$$

Assim, temos $\alpha(k)>1$. 
Para provar a segunda pate do lema, note que se $k_{1} \triangleright k_{2}$, então:

$$
k_{2}^{-1} \circ k_{1} \triangleright I d \Rightarrow \alpha\left(k_{2}^{-1} \circ k_{1}\right)>1 \Rightarrow \alpha\left(k_{2}\right)^{-1} . \alpha\left(k_{1}\right)>1 \Rightarrow \alpha\left(k_{1}\right)>\alpha\left(k_{2}\right),
$$

provando que o morfismo é crescente.

Denote por $T_{t_{h}(k)}$ a translação $x \mapsto x+t_{h}(k)$. Assim, a semi-conjugação $\phi$ descrita após o Lema 3.3.10, satisfaz:

$$
\phi \circ k=T_{t_{h}(k)} \circ \phi
$$

Agora, estamos prontos para o último passo da demonstração do Teorema 3.3.1:

Lema 3.3.13 Para todo $g \in G$, a composição $\phi \circ g$ é uma semi-conjugação com um subgrupo de translações.

Prova. Com efeito, para todo $g \in G$ e todo $k \in \operatorname{ker}\left(\rho_{f}\right)$, temos $g \circ k \circ g^{-1}=\psi_{g}(k)$ e, pela definição de $\phi$, temos:

$$
\phi \circ\left(g \circ k \circ g^{-1}\right)=T_{t_{h}\left(\psi_{g}(k)\right)} \circ \phi=T_{\alpha(g) . t_{h}(k)} \circ \phi .
$$

Então, aplicando $g$ pela direita nos dois lados da equação, temos para todo $g \in G$ e todo $k \in \operatorname{ker}\left(\rho_{f}\right)$

$$
(\phi \circ g) \circ k=T_{\alpha(g) \cdot t_{h}(k)} \circ(\phi \circ g)
$$

Portanto, para todo $k \in \operatorname{ker}\left(\rho_{f}\right)$ a composição $\phi \circ g$ é uma semi-conjugação entre $k$ e uma translação, o que conclui a prova do lema.

Por fim, pela unicidade da semi-conjugação $\phi$ descrita no Lema 3.1.9, temos que para todo $g \in G$, existem $a(g) \in \mathbb{R}$ positivo, e $b(g) \in \mathbb{R}$, com

$$
\phi \circ g=a(g) \cdot \phi+b(g)
$$

Isso significa que para todo $g \in G$, a aplicação $\phi$ induz uma semi-conjugação de $g$ para a aplicação $x \mapsto a(g) \cdot x+b(g)$, como ilustrado no diagrama.

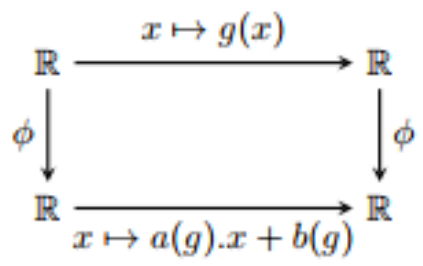

Portanto, $\phi$ é uma semi-conjugação de $G$ com um grupo de aplicações afins, concluindo a prova do Teorema de Solodov. 


\section{4}

\section{Abertura de órbita}

No intuito de estudar e classificar os grupos de homeomorfismos crescentes com no máximo $N$ pontos fixos, é essencial entender seus exemplos. E sem dúvidas, os grupos abelianos fornecem os exemplos mais simples possíveis. Porém, como já vimos no Lema 2.2.28, temos que nesse caso todos os pontos fixos são globais e isso torna a dinâmica da ação trivial demais para agradar qualquer estudioso.

Observamos que os exemplos clássicos, como o grupo das translações e o grupo das aplicações afins, são extremamente importantes para a classificação dos casos $N \leq 1$. Infelizmente, os casos $N \geq 2$ não possuem exemplos clássicos, e isso deixa a busca por grupos não-abelianos com no máximo $N \geq 2$ pontos fixos muito instigante, e nos leva ao tema desse capítulo.

Visando construir tais exemplos, vamos apresentar o conceito de abertura de órbita, que consiste em a partir de uma ação de grupos construir uma semiconjugação que abre um conjunto invariante em intervalos, ou seja, a préimagem de cada ponto do conjunto de abertura pela semi-conjugação é um intervalo compacto. E em seguida, para cada um dos intervalos assim obtidos, vamos incluir uma nova ação.

Dessa forma, um elemento que fixava um dos pontos que foram abertos vai passar a fixar os extremos de um intervalo, além de também fixar os pontos fixos da ação incluída nesse intervalo. Desta forma, criamos novos pontos fixos de forma controlada. A partir desta técnica de abertura, vamos construir exemplos de grupos de homeomorfismos crescentes não-abelianos para os casos $N \geq 2$.

\section{1}

\section{Existência da abertura de uma órbita}

Vamos nesta seção, apresentar o Teorema de Abertura de Órbita, que irá garantir a existência de uma semi-conjugação que abre um conjunto invariante, isto é, uma aplicação que tem como pré-imagem de cada ponto do conjunto um intervalo compacto. Além disso, mostraremos que toda semi-conjugação pode ser vista como uma abertura de órbita, ou seja, os conceitos de abertura de órbita e de semi-conjugação são essencialmente equivalentes. 
Definição 4.1.1 Dados um conjunto $X$ e um grupo $G$ que age sobre $X$, dizemos que dois pontos $x, y \in X$ estão na mesma classe de órbitas se existe $g \in G$, tal que $g(x)=y$. Ou, equivalentemente, $G . x=G . y$.

Observação 4.1.2 Note que a relação $x \sim y \Leftrightarrow G . x=G . y$ é uma relação de equivalência, e portanto as classes de órbitas são classes de equivalência. Como consequência, podemos tomar representantes para cada classe. Isto é, elementos $\left\{x_{k}\right\}_{k \in \Omega} \subset X$, tais que

$$
\bigcup_{k \in \Omega} G \cdot x_{k}=X \text { e, para } k_{1} \neq k_{2} \text { temos } G \cdot x_{k_{1}} \cap G \cdot x_{k_{2}}=\emptyset .
$$

Teorema 4.1.3 (Abertura de Órbita) Sejam $G \subset$ Homeo $_{+}(\mathbb{R})$ um grupo atuando em $\mathbb{R}$ e $A \subset \mathbb{R}$ um subconjunto $G$-invariante enumerável. Considere representantes das classes de órbitas $\left\{x_{k}\right\}_{k \in \Omega} \subset A$ e $A=\bigsqcup_{k \in \Omega} G . x_{k}$.

Para cada $k \in \Omega$ considere $H_{k}$ o estabilizador de $x_{k}$ e ações de grupo $\phi_{k}: H_{k} \rightarrow$ Homeo $_{+}([0,1])$. Então existem uma ação $\Psi: G \rightarrow \mathrm{Homeo}_{+}(\mathbb{R})$ e uma aplicação $h: \mathbb{R} \rightarrow \mathbb{R}$ contínua, crescente e sobrejetiva com as seguintes propriedades:

- h é semi-conjugação da ação $\Psi$ com a ação natural de $G$ : para todo $g \in G$ e todo $x \in \mathbb{R}$ se verifica

$$
h \circ \psi(g)(x)=g \circ h(x) .
$$

Ou seja, o diagrama abaixo comuta

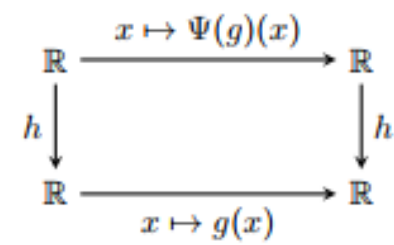

- $h^{-1}(x)$ é reduzida a um único ponto, se $x \notin A$.

- $h^{-1}(x)$ é um intervalo compacto não-trivial, se $x \in A$.

- Para cada $k \in \Omega, \Psi\left(H_{k}\right)$ é o estabilizador de $h^{-1}\left(x_{k}\right)$. E a restrição, $\left.\Psi\left(H_{k}\right)\right|_{h^{-1}\left(x_{k}\right)}$ é conjugada à ação $\phi_{k}$. Ou seja, existe $t_{k}:[0,1] \rightarrow h^{-1}\left(x_{k}\right)$ homeomorfismo crescente tal que para todo $x \in h^{-1}\left(x_{k}\right)$ e todo $w \in H_{k}$, se verifica

$$
\Psi(w)(x)=t_{k} \circ \phi_{k}(w) \circ t_{k}^{-1}(x)
$$


Definição 4.1.4 Dizemos que a ação de grupos $\Psi(G): G \times \mathbb{R} \rightarrow \mathbb{R}$, definida por $(g, x) \mapsto \Psi(g)(x)$ é obtida a partir de $G$, abrindo as órbitas de $\left\{x_{k}\right\}_{k \in \Omega} e$ incluindo $\left\{\phi_{k}\right\}_{k \in \Omega}$ no intervalo.

A prova do Teorema 4.1.3 será uma consequência direta das próximas três proposições. Elas serão divididas em duas subseções: Abertura do conjunto $A$ e extensão da ação para $\mathbb{R}$.

\subsection{1}

\section{Abertura do conjunto $A$}

Proposição 4.1.5 Dados $G \subset$ Homeo $_{+}(\mathbb{R})$ grupo atuando em $\mathbb{R}$ e $A \subset \mathbb{R}$ subconjunto enumerável, G-invariante, existe $h: \mathbb{R} \rightarrow \mathbb{R}$ crescente, contínua e sobrejetiva, tal que:

(1) $h^{-1}(x)$ é um único ponto, se $x \notin A$.

(2) $h^{-1}\left(a_{n}\right)=\left[\alpha_{n}, \beta_{n}\right]$ é um intervalo compacto, se $a_{n} \in A$. Além disso, se $a_{n}<a_{m}$ então $\beta_{n}<\alpha_{m}$.

(3) Seja $X=\mathbb{R} \backslash h^{-1}(A)$ então $\psi: G \rightarrow \operatorname{Bij}_{+}(X)$ definida por

$$
\psi(g)(x)=h^{-1} \circ g \circ h(x)
$$

é uma ação de grupos.

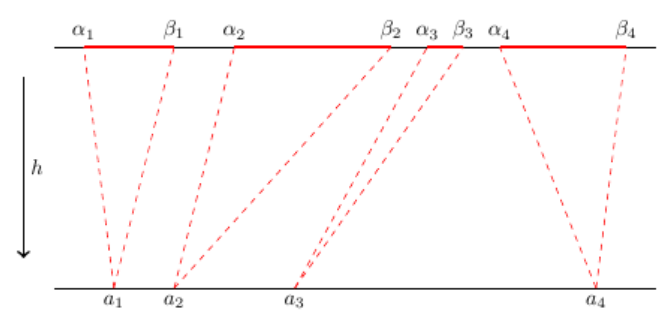

Prova. Pelo Lema 2.1.24, como $A$ é enumerável, existe $h: \mathbb{R} \rightarrow \mathbb{R}$ crescente, contínua e sobrejetiva, que satisfaz os dois primeiros itens. Vamos mostrar que esse mesmo $h$ satisfaz a terceira propriedade também. Para isso primeiro note que

$$
X=\mathbb{R} \backslash h^{-1}(A)=h^{-1}(\mathbb{R}) \backslash h^{-1}(A)=h^{-1}(\mathbb{R} \backslash A)=h^{-1}\left(A^{c}\right) .
$$

Portanto, $h(X)=A^{c}$.

Afirmação. Dado $g \in G$, a aplicação $\psi(g): X \rightarrow X$ é função bijetiva crescente e está bem definida.

Prova. Basta notar que $h: X \rightarrow A^{c}$ é bijeção, pelo item 1. E portanto, $h^{-1}: A^{c} \rightarrow X$ é função bijetiva bem definida. Além disso, como $A$ é $G-$ invariante, então $g: A^{c} \rightarrow A^{c}$ é bijeção bem definida. Temos assim: 


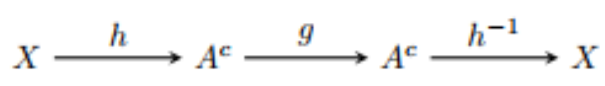

Portanto, $h^{-1} \circ g \circ h(x)=\psi(g)$ é função bijetiva bem definida.

Agora, como $h: X \rightarrow A^{c}$ e $g \circ h: X \rightarrow A^{c}$ são funções crescentes e $h^{-1} \circ g \circ h: X \rightarrow X$ é função bem definida, pelo Corolário 2.1.7, temos que $h^{-1} \circ g \circ h$ é crescente.

O fim da prova da proposição segue da seguinte afirmação.

Afirmação. $\psi: G \rightarrow \mathrm{Bij}_{+}(X)$ é ação de grupos.

Prova. Vamos calcular $\psi\left(g_{1}\right) \circ \psi\left(g_{2}\right)(x)$, lembrando neste domínio a função $h$ é bijetiva

$$
\begin{gathered}
\psi\left(g_{1}\right) \circ \psi\left(g_{2}\right)(x)=h^{-1} \circ g_{1} \circ h \circ h^{-1} \circ g_{2} \circ h(x) \\
=h^{-1} \circ g_{1} \circ g_{2} \circ h(x)=\psi\left(g_{1} \circ g_{2}\right)(x) .
\end{gathered}
$$

E também é claro que: $\psi(I d)(x)=I d$.

Portanto, $h$ satisfaz os três itens do enunciado, como desejado e a prova da proposição está concluída.

Proposição 4.1.6 Existe uma única ação de grupos $\bar{\psi}: G \rightarrow \operatorname{Bij}_{+}(\bar{X})$, que é uma extensão de $\psi$ ao fecho de $X$.

Prova. Dado $a_{n} \in A$, considere o intervalo $h^{-1}\left(a_{n}\right)=\left[\alpha_{n}, \beta_{n}\right]$. Note que

$$
\bar{X}=X \cup \bigcup_{n \in \mathbb{N}}\left\{\alpha_{n}\right\} \cup\left\{\beta_{n}\right\}
$$

Por outro lado, temos as seguintes descrições de $\alpha_{n}$ e $\beta_{n}$ :

$$
\begin{gathered}
\alpha_{n}=\sup \left\{h^{-1}(x) \mid x \notin A, x<a_{n}\right\}, \\
\beta_{n}=\inf \left\{h^{-1}(x) \mid x \notin A, x>a_{n}\right\} .
\end{gathered}
$$

Tome $g \in G$ e seja $a_{m} \in A$, tal que $g\left(a_{n}\right)=a_{m}$. Para que $\bar{\psi}(g)$ seja uma extensão crescente, é necessário que

$$
\begin{gathered}
\bar{\psi}(g)\left(\alpha_{n}\right) \geq \sup \left\{\bar{\psi}(g)\left(h^{-1}(x)\right) \mid x \notin A, x<a_{n}\right\} \\
=\sup \left\{\psi(g)\left(h^{-1}(x)\right) \mid x \notin A, x<a_{n}\right\} \\
=\sup \left\{h^{-1} \circ g \circ h\left(h^{-1}(x)\right) \mid x \notin A, x<a_{n}\right\} \\
=\sup \left\{h^{-1}(g(x)) \mid x \notin A, x<a_{n}\right\} \\
=\sup \left\{h^{-1}(y) \mid y \notin A, y<a_{m}\right\}=\alpha_{m} .
\end{gathered}
$$


E, de forma análoga,

$$
\begin{gathered}
\bar{\psi}(g)\left(\beta_{n}\right) \leq \inf \left\{\bar{\psi}(g)\left(h^{-1}(x)\right) \mid x \notin A, x>a_{n}\right\} \\
=\inf \left\{\psi(g)\left(h^{-1}(x)\right) \mid x \notin A, x>a_{n}\right\} \\
=\inf \left\{h^{-1} \circ g \circ h\left(h^{-1}(x)\right) \mid x \notin A, x>a_{n}\right\} \\
=\inf \left\{h^{-1}(g(x)) \mid x \notin A, x>a_{n}\right\} \\
=\inf \left\{h^{-1}(y) \mid y \notin A, y>a_{m}\right\}=\beta_{m} .
\end{gathered}
$$

Portanto,

$$
\alpha_{m} \leq \bar{\psi}(g)\left(\alpha_{n}\right)<\bar{\psi}(g)\left(\beta_{n}\right) \leq \beta_{m} .
$$

E como, $\left(\alpha_{m}, \beta_{m}\right) \cap \bar{X}=\emptyset$, temos $\bar{\psi}(g)\left(\alpha_{n}\right)=\alpha_{m}$ e $\bar{\psi}(g)\left(\beta_{n}\right)=\beta_{m}$. Assim o único candidato possível para extensão da ação $\psi$ deve ser da forma:

$$
\bar{\psi}(g)(x)=\left\{\begin{aligned}
h^{-1} \circ g \circ h(x) & \text { se } x \in X \\
\alpha_{m} & \text { se } x=\alpha_{n} \text { e } g\left(a_{n}\right)=a_{m} \\
\beta_{m} & \text { se } x=\beta_{n} \text { e } g\left(a_{n}\right)=a_{m}
\end{aligned}\right.
$$

Afirmação. A aplicação $\bar{\psi}: G \rightarrow \operatorname{Bij}_{+}(\bar{X})$ definida acima é uma ação de grupos.

Prova. Pela construção de $\bar{\psi}(g)\left(\alpha_{n}\right)$ e $\bar{\psi}(g)\left(\beta_{n}\right)$, e por $\bar{\psi}$ ser extensão de $\psi$ em $X$, fica claro que

$$
\bar{\psi}(g): \bar{X} \rightarrow \bar{X}
$$

é bijetiva crescente, para todo $g \in G$.

Para provar que é ação de grupos, basta verificar as propriedades para $x \in \bar{X} \backslash X$. Com efeito, sejam $a_{n} \in A$ e $g_{2}\left(a_{n}\right)=a_{m}, g_{1}\left(a_{m}\right)=a_{k}$, e portanto $g_{1} \circ g_{2}\left(a_{n}\right)=a_{k}$. Temos:

$$
\begin{aligned}
& \bar{\psi}\left(g_{1}\right) \circ \bar{\psi}\left(g_{2}\right)\left(\alpha_{n}\right)=\bar{\psi}\left(g_{1}\right)\left(\alpha_{m}\right)=\alpha_{k}=\bar{\psi}\left(g_{1} \circ g_{2}\right)\left(\alpha_{n}\right), \\
& \bar{\psi}\left(g_{1}\right) \circ \bar{\psi}\left(g_{2}\right)\left(\beta_{n}\right)=\bar{\psi}\left(g_{1}\right)\left(\beta_{m}\right)=\beta_{k}=\bar{\psi}\left(g_{1} \circ g_{2}\right)\left(\beta_{n}\right) .
\end{aligned}
$$

Além disso, tomando $g=I d$, temos $\operatorname{Id}\left(a_{n}\right)=a_{n}$, e assim

$$
\bar{\psi}(I d)\left(\alpha_{n}\right)=\alpha_{n} \text { e } \bar{\psi}(I d)\left(\beta_{n}\right)=\beta_{n}
$$

Verificando que $\bar{\psi}$ é ação de grupos bem definida.

Portanto existe uma única extensão $\bar{\psi}$, concluindo assim, a prova da Proposição 4.1.6. 


\subsection{2}

\section{Extensão da ação para $\mathbb{R}$}

Note que, como $A$ é $G$-invariante, então temos $G . a \subset A$, para todo $a \in A$. Sejam $x_{k} \in A$ os representantes de cada órbita em $A$. Isto é,

$$
A=\bigsqcup_{k \in \Omega \subset \mathbb{N}} G \cdot x_{k}
$$

Seja $H_{k} \subset G$ o estabilizador de $x_{k}$, ou seja

$$
H_{k}=\left\{g \in G \mid g\left(x_{k}\right)=x_{k}\right\}
$$

e defina os intervalos compactos

$$
h^{-1}\left(x_{k}\right)=I_{k}=\left[\alpha_{k}^{\prime}, \beta_{k}^{\prime}\right]
$$

Proposição 4.1.7 Para cada $k \in \Omega$ considere $\phi_{k}^{\prime}: H_{k} \rightarrow$ Homeo $_{+}\left(I_{k}\right)$ ações de grupo. Então existe uma $\Psi: G \rightarrow$ Homeo $_{+}(\mathbb{R})$ que é uma ação de grupos bem definida, tal que:

- $\Psi(g)(x)=\bar{\psi}(g)(x)$ para todo $x \in \bar{X}$.

- $\Psi\left(H_{k}\right)$ é o estabilizador de $I_{k}$ e a restrição $\left.\Psi\left(H_{k}\right)\right|_{I_{k}}$ é equivalente à ação $\phi_{k}^{\prime}$, isto é, para todo $w \in H_{k}$ e todo $x \in I_{k}$

$$
\Psi(w)(x)=\phi_{k}^{\prime}(w)(x)
$$

- h é uma semi-conjugação da ação $\Psi$ com a ação natural de $G$, satisfazendo para todo $g \in G$ e todo $x \in \mathbb{R}$,

$$
h \circ \Psi(g)(x)=g \circ h(x) .
$$

Ou seja, o diagrama é comutativo

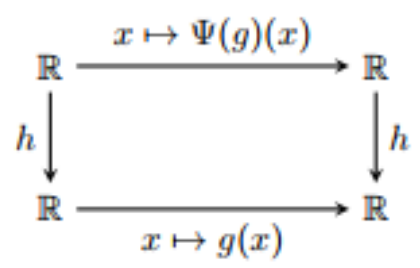

Prova. Para todo $a_{n} \in A$, existe um único $x_{k}$, com

$$
a_{n} \in G \cdot x_{k}
$$


Para cada $a_{n} \in A$ que não é o representante da sua órbita (i.e. $a_{n} \neq x_{k}$ para todo $k \in \Omega$ ) escolha um elemento $f_{n} \in G$, tal que:

$$
f_{n}\left(a_{n}\right)=x_{k}
$$

Note que: $\bar{\psi}\left(f_{n}\right)\left(\alpha_{n}\right)=\alpha_{k}^{\prime}$ e $\bar{\psi}\left(f_{n}\right)\left(\beta_{n}\right)=\beta_{k}^{\prime}$. Observe que se a extensão $\Psi$ existir, necessariamente deve verificar

$$
\Psi\left(f_{n}\right)\left(\left[\alpha_{n}, \beta_{n}\right]\right)=\left[\alpha_{k}^{\prime}, \beta_{k}^{\prime}\right]
$$

Vamos portanto, definir para todo $a_{n} \in A$ e todo $x \in\left[\alpha_{n}, \beta_{n}\right]$

$$
\Psi\left(f_{n}\right)(x):=L_{n}(x)
$$

onde $L_{n}:\left[\alpha_{n}, \beta_{n}\right] \rightarrow\left[\alpha_{k}^{\prime}, \beta_{k}^{\prime}\right]$ é a aplicação afim que transforma, de forma bijetiva e crescente, um intervalo no outro.

Fixe agora, $g \in G$ e $x \notin \bar{X}$ quaisquer. Note que,

$$
x \notin \bar{X} \Rightarrow x \in\left(h^{-1}(A)\right)^{\mathrm{o}}=\bigsqcup_{n \in \mathbb{N}}\left(\alpha_{n}, \beta_{n}\right) .
$$

Portanto, existe um único $a_{n} \in A$ tal que $x \in\left(\alpha_{n}, \beta_{n}\right)$.

Seja $g\left(a_{n}\right)=a_{m} \in A$, existe um único representante $x_{k}$ tal que: $a_{n}, a_{m} \in G . x_{k}$. Agora, note que

$$
f_{n}\left(a_{n}\right)=x_{k} \text { e } f_{m}\left(a_{m}\right)=x_{k} \Rightarrow f_{m} \circ g \circ f_{n}^{-1}\left(x_{k}\right)=x_{k}
$$

Portanto, $f_{m} \circ g \circ f_{n}^{-1} \in H_{k}$. Porém, para respeitar o segundo item da proposição, é necessário que

$$
\Psi\left(f_{m} \circ g \circ f_{n}^{-1}\right)(x)=\phi_{k}^{\prime}\left(f_{m} \circ g \circ f_{n}^{-1}\right)(x) .
$$

Mas, para que $\Psi$ seja ação de grupos, é necessário que

$$
\Psi\left(f_{m} \circ g \circ f_{n}^{-1}\right)(x)=\Psi\left(f_{m}\right) \circ \Psi(g) \circ \Psi\left(f_{n}\right)^{-1}(x) .
$$

Juntando as duas equações acima, temos:

$$
\Psi(g)(x)=\Psi\left(f_{m}\right)^{-1} \circ \phi_{k}^{\prime}\left(f_{m} \circ g \circ f_{n}^{-1}\right) \circ \Psi\left(f_{n}\right)(x) .
$$

Lembrando que $\Psi\left(f_{n}\right)(x)=L_{n}(x)$, para todo $a_{n} \in A$ e todo $x \in\left[\alpha_{n}, \beta_{n}\right]$, 
concluímos

$$
\Psi(g)(x)=L_{m}^{-1} \circ \phi_{k}^{\prime}\left(f_{m} \circ g \circ f_{n}^{-1}\right) \circ L_{n}(x) .
$$

Afirmação. Seja $\Psi: G \rightarrow \operatorname{Homeo}_{+}(\mathbb{R})$, definido para cada $\left(\alpha_{n}, \beta_{n}\right)$, por:

$$
\Psi(g)(x):=\left\{\begin{aligned}
\bar{\psi}(g)(x) & \text { se } x \in \bar{X} \\
L_{m}^{-1} \circ \phi_{k}^{\prime}\left(f_{m} \circ g \circ f_{n}^{-1}\right) \circ L_{n}(x) & \text { se } x \in\left(\alpha_{n}, \beta_{n}\right)
\end{aligned}\right.
$$

onde $a_{n} \in G$. $x_{k}$ e $g\left(a_{n}\right)=a_{m}$. É uma ação de grupos bem definida.

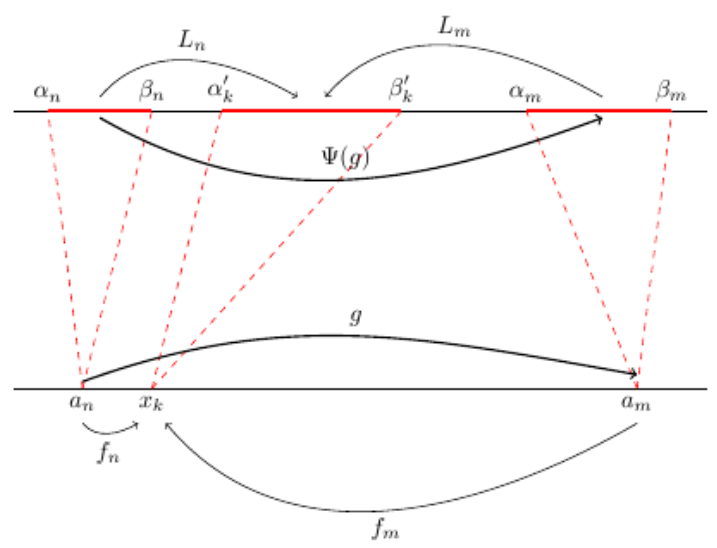

Prova. É fácil notar que $\Psi(g)$ é bijeção crescente em cada intervalo $\left[\alpha_{n}, \beta_{n}\right]$. E como:

$$
\left.\Psi(g)\right|_{\bar{X}}=\bar{\psi}(g) \in \operatorname{Bij}_{+}(\bar{X}),
$$

concluímos que $\Psi(g) \in \mathrm{Bij}_{+}(\mathbb{R})$. Portanto, pelo Lema 2.1.13, temos que $\Psi(g)$ é um homeomorfismo crescente.

Agora, vamos calcular $\Psi\left(g_{1}\right) \circ \Psi\left(g_{2}\right)(x)$. Temos que considerar dois casos:

- Caso $x \in \bar{X}$ :

$$
\Psi\left(g_{1}\right) \circ \Psi\left(g_{2}\right)(x)=\Psi\left(g_{1}\right)\left(\bar{\psi}\left(g_{2}\right)(x)\right)
$$

lembrando que $\bar{\psi}\left(g_{2}\right)(x) \in \bar{X}$, temos:

$$
\begin{gathered}
\Psi\left(g_{1}\right)\left(\bar{\psi}\left(g_{2}\right)(x)\right)=\bar{\psi}\left(g_{1}\right)\left(\bar{\psi}\left(g_{2}\right)(x)\right) \\
=\bar{\psi}\left(g_{1} \circ g_{2}\right)(x)=\Psi\left(g_{1} \circ g_{2}\right)(x) .
\end{gathered}
$$

- Caso $x \in\left(\alpha_{n}, \beta_{n}\right)$ :

Seja $g_{2}\left(a_{n}\right)=a_{m}$ e $g_{1}\left(a_{m}\right)=a_{l}$, e assim: $\left(g_{1} \circ g_{2}\right)\left(a_{n}\right)=a_{l}$. E seja $x_{k}$ o representante da órbita de $a_{n}$, e consequentemente, das órbitas de $a_{m} \mathrm{e}$ $a_{l}$ também. Lembre que $\Psi\left(g_{2}\right)(x) \in\left(\alpha_{m}, \beta_{m}\right)$. Assim, temos: 


$$
\begin{gathered}
\Psi\left(g_{1}\right) \circ \Psi\left(g_{2}\right)(x)=\Psi\left(g_{1}\right)\left(\Psi\left(g_{2}\right)(x)\right) \\
=L_{l}^{-1} \circ \phi_{k}^{\prime}\left(f_{l} \circ g_{1} \circ f_{m}^{-1}\right) \circ L_{m}\left(\Psi\left(g_{2}\right)(x)\right) \\
=L_{l}^{-1} \circ \phi_{k}^{\prime}\left(f_{l} \circ g_{1} \circ f_{m}^{-1}\right) \circ L_{m} \circ L_{m}^{-1} \circ \phi_{k}^{\prime}\left(f_{m} \circ g_{2} \circ f_{n}^{-1}\right) \circ L_{n}(x) \\
=L_{l}^{-1} \circ \phi_{k}^{\prime}\left(f_{l} \circ g_{1} \circ f_{m}^{-1}\right) \circ \phi_{k}^{\prime}\left(f_{m} \circ g_{2} \circ f_{n}^{-1}\right) \circ L_{n}(x) .
\end{gathered}
$$

Porém, $\phi_{k}^{\prime}: I_{k} \rightarrow I_{k}$ é ação de grupos, então:

$$
\begin{gathered}
=L_{l}^{-1} \circ \phi_{k}^{\prime}\left(f_{l} \circ g_{1} \circ f_{m}^{-1} \circ f_{m} \circ g_{2} \circ f_{n}^{-1}\right) \circ L_{n}(x) \\
=L_{l}^{-1} \circ \phi_{k}^{\prime}\left(f_{l} \circ g_{1} \circ g_{2} \circ f_{n}^{-1}\right) \circ L_{n}(x)=\Psi\left(g_{1} \circ g_{2}\right)(x) .
\end{gathered}
$$

Portanto, acabamos de provar que $\Psi\left(g_{1}\right) \circ \Psi\left(g_{2}\right)=\Psi\left(g_{1} \circ g_{2}\right)$. Falta verificar que $\Psi(I d)=I d$. Novamente, temos dois casos:

- Caso $x \in \bar{X}$ :

$$
\Psi(I d)(x)=\bar{\psi}(I d)(x)=I d(x)=x
$$

- Caso $x \in\left(\alpha_{n}, \beta_{n}\right)$ :

$$
\begin{gathered}
\Psi(I d)(x)=L_{n}^{-1} \circ \phi_{k}^{\prime}\left(f_{n} \circ I d \circ f_{n}^{-1}\right) \circ L_{n}(x) \\
=L_{n}^{-1} \circ \phi_{k}^{\prime}(I d) \circ L_{n}(x)=L_{n}^{-1} \circ I d \circ L_{n}(x)=I d(x)=x .
\end{gathered}
$$

Concluindo a prova da afirmação.

A ação $\Psi: G \rightarrow$ Homeo $_{+}(\mathbb{R})$ definida na afirmação anterior, claramente satisfaz os dois primeiros itens da Proposição 4.1.7. Para o terceiro item, basta observar que:

- Caso $x \in X$,

$$
\Psi(g)(x)=\bar{\psi}(g)(x)=\psi(g)(x)=h^{-1} \circ g \circ h(x) .
$$

E assim, como $h$ é bijetiva em $X$

$$
h \circ \Psi(g)(x)=h \circ h^{-1} \circ g \circ h(x)=g \circ h(x) .
$$

- Caso $x \notin X$, então existe $a_{n} \in A$ com:

$$
x \in\left[\alpha_{n}, \beta_{n}\right]=h^{-1}\left(a_{n}\right) .
$$


Seja $g\left(a_{n}\right)=a_{m}$, temos que

$$
\Psi(x) \in\left[\Psi\left(\alpha_{n}\right), \Psi\left(\beta_{n}\right)\right]=\left[\alpha_{m}, \beta_{m}\right]=h^{-1}\left(a_{m}\right) .
$$

Portanto,

$$
h(\Psi(x))=a_{m}=g\left(a_{n}\right)=g(h(x))=g \circ h(x) .
$$

Concluímos assim, a prova da proposição.

Portanto, a prova do Teorema 4.1.3 está concluída.

A próxima observação mostra que os conceitos de semi-conjugação e de abertura de órbita são equivalentes.

Observação 4.1.8 Toda semi-conjugação pode ser descrita como uma abertura de órbita. Mais precisamente:

Sejam $G^{\prime}, G \subset$ Homeo $_{+}(\mathbb{R})$ dois grupos agindo sobre $\mathbb{R}$ e $h: \mathbb{R} \rightarrow \mathbb{R}$ aplicação contínua, crescente e sobrejetiva, tal que $G^{\prime}$ é semi-conjugada a $G$ por h. Isto é, para todo $g^{\prime} \in G^{\prime}$ existe $g \in G$, satisfazendo:

$$
h \circ g^{\prime}(x)=g \circ h(x), \text { para todo } x \in \mathbb{R} .
$$

Além disso, sejam $\left\{x_{k}\right\}_{k \in \Omega}$ representantes das classes de órbitas em $G$ de

$$
A:=\left\{x \in \mathbb{R} \mid h^{-1}(x) \text { é um intervalo não-trivial }\right\}
$$

e $H_{k} \subset G$ o estabilizador de $x_{k}$. Note que $H_{k}=\left.G^{\prime}\right|_{h^{-1}\left(x_{k}\right)}$, e defina para todo $k \in \Omega$, as ações de grupos $\phi_{k}: H_{k} \times h^{-1}\left(x_{k}\right) \rightarrow h^{-1}\left(x_{k}\right)$ por:

$$
\phi_{k}(w):=\left.w^{\prime}\right|_{h^{-1}\left(x_{k}\right)} \text {, para todo } w \in H_{k} .
$$

Então, por definição, $G^{\prime}$ é obtida a partir de $G$, abrindo as órbitas de $\left\{x_{k}\right\}_{k \in \Omega}$ e incluindo $\left\{\phi_{k}\right\}_{k \in \Omega}$ no intervalo.

\section{2}

\section{Unicidade da construção}

A construção da abertura de órbita feita na seção anterior, pode deixar o leitor um pouco confuso. Afinal, foram tantas escolhas árbitrarias, que poderiamos ter dois grupos obtidos pela Abertura de Órbita, e eles não terem nada em comum, fazendo da construção, algo vago demais para ser usado de forma bem definida. Porém, esta seção vem para trazer segurança ao leitor e dar credibilidade para construção feita, pois nela será demonstrada a "unicidade" 
da abertura de órbita, concluindo que duas ações de grupos obtidas a partir de um mesmo grupo e incluindo as mesmas ações no intervalo, são conjugadas. Esse é o conteúdo do próximo teorema.

Teorema 4.2.1 Se $\theta_{1}$ e $\theta_{2}$ são duas ações de grupos, ambas obtidas a partir de $G$, abrindo as órbitas de $\left\{x_{k}\right\}_{k \in \Omega}$ e incluindo $\left\{\phi_{k}\right\}_{k \in \Omega}$ no intervalo, então $\theta_{1}$ e $\theta_{2}$ são conjugadas por um homeomorfismo crescente.

Prova. Sejam $h_{1}$ e $h_{2}$ as semi-conjugações de $\theta_{1}$ e $\theta_{2} \operatorname{com} G$, assim como no Teorema 4.1.3. E seja:

$$
A:=\bigsqcup_{k \in \Omega \subset \mathbb{N}} G \cdot x_{k}
$$

Observe que $A$ é um subconjunto enumerável, $G$-invariante e que para cada $a_{n} \in A$, existe um único $x_{k}$, tal que $a_{n} \in G . x_{k}$.

Sejam, para cada $a_{n} \in A$

$$
h_{1}^{-1}\left(a_{n}\right)=\left[\alpha_{n}, \beta_{n}\right] \text { e } h_{2}^{-1}\left(a_{n}\right)=\left[\tilde{\alpha}_{n}, \tilde{\beta}_{n}\right]
$$

os intervalos compactos, descritos no Teorema 4.1.3.

Seja $t_{k}:[0,1] \rightarrow h_{1}^{-1}\left(x_{k}\right)$ um homeomorfismo crescente que satisfaz, para todo $x \in h_{1}^{-1}\left(x_{k}\right)$ e todo $w \in H_{k}$

$$
\theta_{1}(w)(x)=t_{k} \circ \phi_{k}(w) \circ t_{k}^{-1}(x)
$$

E $\tilde{t}_{k}:[0,1] \rightarrow h_{2}^{-1}\left(x_{k}\right)$ um homeomorfismo crescente que satisfaz, para todo $x \in h_{2}^{-1}\left(x_{k}\right)$ e todo $w \in H_{k}$

$$
\theta_{2}(w)(x)=\tilde{t}_{k} \circ \phi_{k}(w) \circ \tilde{t}_{k}^{-1}(x)
$$

Fixe, para cada $a_{n} \in A, f_{n} \in G$ tais que $f_{n}\left(a_{n}\right)=x_{k}$. Defina agora:

$$
\Gamma(x)=\left\{\begin{aligned}
h_{2}^{-1} \circ h_{1}(x) & \text { se } x \notin h_{1}^{-1}(A), \\
\theta_{2}\left(f_{n}^{-1}\right) \circ \tilde{t}_{k} \circ t_{k}^{-1} \circ \theta_{1}\left(f_{n}\right)(x) & \text { se } x \in\left[\alpha_{n}, \beta_{n}\right] .
\end{aligned}\right.
$$

Observe que $\Gamma$ define uma aplicação de $\mathbb{R}$ em $\mathbb{R}$.

Afirmação. A aplicação $\Gamma: \mathbb{R} \rightarrow \mathbb{R}$ é um homeomorfismo crescente.

Prova. Por definição, $h_{1}:\left(h_{1}^{-1}(A)\right)^{c} \rightarrow(A)^{c}$ e $h_{2}^{-1}:(A)^{c} \rightarrow\left(h_{2}^{-1}(A)\right)^{c}$ são funções bijetivas e crescentes. Logo,

$$
h_{2}^{-1} \circ h_{1}:\left(h_{1}^{-1}(A)\right)^{c} \rightarrow\left(h_{2}^{-1}(A)\right)^{c}
$$


também é bijetiva e crescente. Como $f_{n}\left(a_{n}\right)=x_{k}$, temos que:

$$
\begin{gathered}
\theta_{1}\left(f_{n}\right):\left[\alpha_{n}, \beta_{n}\right] \rightarrow h_{1}^{-1}\left(x_{k}\right), \\
t_{k}^{-1}: h_{1}^{-1}\left(x_{k}\right) \rightarrow[0,1], \\
\tilde{t}_{k}:[0,1] \rightarrow h_{2}^{-1}\left(x_{k}\right), \\
\theta_{2}\left(f_{n}^{-1}\right): h_{2}^{-1}\left(x_{k}\right) \rightarrow\left[\tilde{\alpha}_{n}, \tilde{\beta}_{n}\right],
\end{gathered}
$$

são todas aplicações bijetivas e crescentes. Logo,

$$
\theta_{2}\left(f_{n}^{-1}\right) \circ \tilde{t}_{k} \circ t_{k}^{-1} \circ \theta_{1}\left(f_{n}\right):\left[\alpha_{n}, \beta_{n}\right] \rightarrow\left[\tilde{\alpha}_{n}, \tilde{\beta}_{n}\right]
$$

é bijetiva e crescente. Portanto, é claro que $\Gamma: \mathbb{R} \rightarrow \mathbb{R}$ é bijetiva.

Para concluir que é crescente, basta observar que: $\Gamma\left(\alpha_{n}\right)=\tilde{\alpha}_{n}$ Porém, $\alpha_{n}$ pode ser descrito como:

$$
\alpha_{n}=\sup \left\{h_{1}^{-1}(x) \mid x \notin A, x<a_{n}\right\}
$$

Portanto:

$$
\begin{gathered}
\Gamma\left(\alpha_{n}\right)=\tilde{\alpha}_{n}=\sup \left\{h_{2}^{-1}(x) \mid x \notin A, x<a_{n}\right\} \\
=\sup \left\{h_{2}^{-1} \circ h_{1} \circ h_{1}^{-1}(x) \mid x \notin A, x<a_{n}\right\} \\
=\sup \left\{\Gamma \circ h_{1}^{-1}(x) \mid x \notin A, x<a_{n}\right\} .
\end{gathered}
$$

E assim,

$$
\Gamma\left(\sup \left\{h_{1}^{-1}(x) \mid x \notin A, x<a_{n}\right\}\right)=\sup \left\{\Gamma \circ h_{1}^{-1}(x) \mid x \notin A, x<a_{n}\right\} .
$$

Analogamente para $\beta_{n}$ :

$$
\Gamma\left(\inf \left\{h_{1}^{-1}(x) \mid x \notin A, x>a_{n}\right\}\right)=\inf \left\{\Gamma \circ h_{1}^{-1}(x) \mid x \notin A, x>a_{n}\right\} .
$$

As duas igualdades acima, só podem ser verdade se $\Gamma$ for crescente também entre elementos de $\left[\alpha_{n}, \beta_{n}\right]$ e $h_{1}^{-1}(A)$. Portanto, $\Gamma$ é bijeção crescente. Concluímos, pelo Lema 2.1.13, que $\Gamma: \mathbb{R} \rightarrow \mathbb{R}$ é homeomorfismo crescente.

Afirmação. A aplicação $\Gamma: \mathbb{R} \rightarrow \mathbb{R}$ é uma conjugação entre $\theta_{1}$ e $\theta_{2}$. Isto é, para todo $g \in G$ e todo $x \in \mathbb{R}$ se verifica

$$
\Gamma \circ \theta_{1}(g)(x)=\theta_{2}(g) \circ \Gamma(x)
$$


Ou seja, o diagrama é comutativo

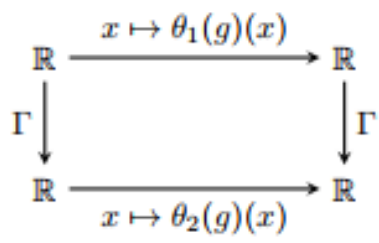

Prova. Temos novamente que considerar dois casos, $x \notin h_{1}^{-1}(A)$ e $x \in\left[\alpha_{n}, \beta_{n}\right]$ para algum $n$.

Caso $x \notin h_{1}^{-1}(A)$, observe que pela definição de $h_{1}$, temos para todo $x \in \mathbb{R}$

$$
h_{1} \circ \theta_{1}(g)(x)=g \circ h_{1}(x) .
$$

Além disso, para $h_{1}(x) \notin A, h_{2}$ é bijetiva, portanto

$$
h_{2}^{-1} \circ g\left(h_{1}(x)\right)=\theta_{2}(g) \circ h_{2}^{-1}\left(h_{1}(x)\right) \text {. }
$$

Lembrando também que $h_{1}^{-1}(A)$ é $\theta_{1}$-invariante, segue que

$$
\begin{gathered}
\Gamma \circ \theta_{1}(g)(x)=h_{2}^{-1} \circ h_{1} \circ \theta_{1}(g)(x)=h_{2}^{-1} \circ g \circ h_{1}(x) \\
=\theta_{2}(g) \circ h_{2}^{-1} \circ h_{1}(x)=\theta_{2}(g) \circ \Gamma(x) .
\end{gathered}
$$

Caso $x \in\left[\alpha_{n}, \beta_{n}\right]$, seja $g\left(a_{n}\right)=a_{m}$ e $x_{k}$ o representante da sua órbita. Então,

$$
f_{m} \circ g \circ f_{n}^{-1}\left(x_{k}\right)=x_{k} \Rightarrow f_{m} \circ g \circ f_{n}^{-1} \in H_{k} .
$$

Portanto,

$$
\theta_{1}\left(f_{m} \circ g \circ f_{n}^{-1}\right)(x)=t_{k} \circ \phi_{k}\left(f_{m} \circ g \circ f_{n}^{-1}\right) \circ t_{k}^{-1}(x) .
$$

E assim, como $\theta_{1}$ é ação de grupos, temos que

$$
\theta_{1}(g)(x)=\theta_{1}\left(f_{m}^{-1}\right) \circ t_{k} \circ \phi_{k}\left(f_{m} \circ g \circ f_{n}^{-1}\right) \circ t_{k}^{-1} \circ \theta_{1}\left(f_{n}\right)(x) .
$$

Vamos calcular $\Gamma \circ \theta_{1}(g)(x)$ :

$$
\begin{gathered}
\Gamma \circ \theta_{1}(g)(x)=\Gamma \circ \theta_{1}\left(f_{m}^{-1}\right) \circ t_{k} \circ \phi_{k}\left(f_{m} \circ g \circ f_{n}^{-1}\right) \circ t_{k}^{-1} \circ \theta_{1}\left(f_{n}\right)(x) \\
=\theta_{2}\left(f_{m}^{-1}\right) \circ \tilde{t}_{k} \circ t_{k}^{-1} \circ \theta_{1}\left(f_{m}\right) \circ \theta_{1}\left(f_{m}^{-1}\right) \circ t_{k} \circ \phi_{k}\left(f_{m} \circ g \circ f_{n}^{-1}\right) \circ t_{k}^{-1} \circ \theta_{1}\left(f_{n}\right)(x) \\
=\theta_{2}\left(f_{m}^{-1}\right) \circ \tilde{t}_{k} \circ t_{k}^{-1} \circ t_{k} \circ \phi_{k}\left(f_{m} \circ g \circ f_{n}^{-1}\right) \circ t_{k}^{-1} \circ \theta_{1}\left(f_{n}\right)(x) \\
=\theta_{2}\left(f_{m}^{-1}\right) \circ \tilde{t}_{k} \circ \phi_{k}\left(f_{m} \circ g \circ f_{n}^{-1}\right) \circ t_{k}^{-1} \circ \theta_{1}\left(f_{n}\right)(x)
\end{gathered}
$$




$$
\begin{gathered}
=\theta_{2}\left(f_{m}^{-1}\right) \circ \tilde{t}_{k} \circ \phi_{k}\left(f_{m} \circ g \circ f_{n}^{-1}\right) \circ \tilde{t}_{k}^{-1} \circ \theta_{2}\left(f_{n}^{-1}\right) \circ \theta_{2}\left(f_{n}\right) \circ \tilde{t}_{k} \circ t_{k}^{-1} \circ \theta_{1}\left(f_{n}\right)(x) \\
=\theta_{2}\left(f_{m}^{-1}\right) \circ \tilde{t}_{k} \circ \phi_{k}\left(f_{m} \circ g \circ f_{n}^{-1}\right) \circ \tilde{t}_{k}^{-1} \circ \theta_{2}\left(f_{n}^{-1}\right) \circ \Gamma(x) \\
=\theta_{2}(g) \circ \Gamma(x),
\end{gathered}
$$

concluindo desta forma a prova da afirmação.

A prova do Teorema 4.2.1 decorre imediatamente das duas afirmações anteriores.

\section{3}

\section{Construção de grupo com no máximo $\mathbf{N}$ pontos fixos}

Nesta seção, vamos aplicar o conceito de Abertura de Órbita e descrever uma maneira de construir exemplos de grupos não-abelianos de homeomorfismos com no máximo $N \geq 2$ pontos fixos.

Além disso, veremos que todos os exemplos serão semi-conjugados a um grupo com no máximo um ponto fixo. Isso vai nos direcionar, na procura por uma classificação para grupos com até dois pontos fixos, assunto que tomará forma no próximo capítulo.

E também, concluiremos que existem restrições dinâmicas, como minimalidade e transitividade da ação, dependendo exclusivamente do número $N$ de pontos fixos. Isto mostra que, mesmo sendo todos semi-conjugados a grupos com no máximo um ponto fixo, existem diferenças intrínsecas na dinâmica das ações.

Teorema 4.3.1 Sejam:

- $G \subset \mathrm{Homeo}_{+}(\mathbb{R})$ com no máximo um ponto fixo,

- um ponto $x_{0} \in \mathbb{R}$ com $G$. $x_{0}$ enumerável e $H \subset G$ seu estabilizador,

- uma ação $\phi: H \rightarrow$ Homeo $_{+}([0,1])$ com no máximo $N$ pontos fixos em $(0,1)$.

Considere $\Psi(G) \subset$ Homeo $_{+}(\mathbb{R})$ uma ação obtida a partir de $G$ abrindo a órbita do ponto $x_{0}$ e incluindo $\phi$ no intervalo, então:

- O estabilizador $H$ é abeliano.

- Todos os pontos fixos dos elementos de $\phi(H)$ são globais para a ação de $\phi(H)$.

- A ação $\Psi(G)$ tem no máximo $N+2$ pontos fixos. Além disso, existe elemento com $N+2$ pontos fixos, se e só se, $H$ é não-trivial e $\phi(H)$ contém um elemento com $N$ pontos fixos no interior do intervalo.

- Se $H$ é não-trivial e $x_{0}$ não é um ponto fixo global da ação de $G$, então o grupo $\Psi(G)$ não é abeliano. 
Prova. Os itens serão separados em afirmações e serão provados um por um, começando por:

Afirmação. O estabilizador $H$ é abeliano.

Prova. Observe que $H \subset G \subset \mathrm{Homeo}_{+}(\mathbb{R})$ é um subgrupo, portanto $H$ também tem no máximo um ponto fixo. Porém, como $H$ é o estabilizador, temos para todo $w \in H, w\left(x_{0}\right)=x_{0}$. Portanto, $H$ age com no máximo um ponto fixo e possui um ponto fixo global. Pelo Teorema de Solodov, $H$ é abeliano.

Afirmação. Todos os pontos fixos de $\phi(H)$ são globais.

Prova. Fixe $t:(0,1) \rightarrow \mathbb{R}$ homeomorfismo crescente e considere a ação $\phi^{\prime}: H \times \mathbb{R} \rightarrow \mathbb{R}$, definida por

$$
\phi^{\prime}(w)(x)=t \circ \phi(w) \circ t^{-1}(x) .
$$

Pelo Lema 2.2.18, como $\phi$ tem no máximo $N$ pontos fixos em $(0,1)$, por conjugação, $\phi^{\prime}$ também tem no máximo $N$ pontos fixos. Porém, $H$ abeliano, então, pelo Lema 2.2.28, todos os pontos fixos de $\phi^{\prime}(H)$ são pontos fixos globais. Assim, por conjugação, todos os pontos fixos de $\phi(H)$ são globais.

Afirmação. $\Psi(G)$ tem no máximo $N+2$ pontos fixos. Além disso, existe elemento com $N+2$ pontos fixos, se e só se, $H$ é não-trivial e $\phi(H)$ possui um elemento com $N$ pontos fixos.

Prova. Lembre que $h \circ \Psi(g)(x)=g \circ h(x)$ e que $g \in G$ possui no máximo um ponto fixo. Portanto, caso $g$ é sem pontos fixos, $\Psi(g)$ também é sem pontos fixos, pelo Lema 2.2.23. Ainda pelo Lema 2.2.23, caso $x \in \mathbb{R}$ é o único ponto fixo de $g$, temos:

$$
\operatorname{Fix}(\Psi(g)) \subset h^{-1}(x) .
$$

Se $x \notin G \cdot x_{0}$, então $h^{-1}(x)$ é um único ponto e portanto $\operatorname{Fix}(\Psi(g))=$ $h^{-1}(x)$, ou seja $\Psi(g)$ possui um ponto fixo.

Se $x \in G$. $x_{0}$, então existe $g \in G$ não-trivial que fixa $x$, se e só se, $H$ é não trivial. Portanto, apenas devemos considerar $H$ não-trivial para este caso.

Suponha então $H$ não trivial e um elemento $\phi(w) \in \phi(H) \operatorname{com} N$ pontos fixos no intervalo. Sabemos que a pré-imagem de $h$ é dada por um intervalo compacto, então considere $h^{-1}(x)=[\alpha, \beta]$, e temos

$$
\Psi(w)([\alpha, \beta])=[\alpha, \beta]
$$

Portanto, necessariamente: $\alpha, \beta \in \operatorname{Fix}(\Psi(w))$.

Além disso, em $[\alpha, \beta]$, temos que $\Psi(w)$ é conjugado a $\phi(w)$ que possui $N$ pontos fixos, e portanto pelo Lema 2.2.18, $\Psi(w)$ possui $N$ pontos fixos 
em $(\alpha, \beta)$, totalizando $N+2$ pontos fixos em $[\alpha, \beta]=h^{-1}(x)$. Mas, como $\operatorname{Fix}(\Psi(w)) \subset h^{-1}(x)$, concluímos, que $\Psi(g)$ possui exatamente $N+2$ pontos fixos. Fica concluída a prova da afirmação.

Afirmação. Se $H$ é não-trivial e $x_{0}$ não é um ponto fixo global da ação de $G$, então o grupo $\Psi(G)$ não é abeliano.

Prova. Observe que como $H$, o estabilizador de $x_{0}$, é não-trivial, segue que existe $h \in H$ não-trivial com $h\left(x_{0}\right)=x_{0}$. Porém, por hipótese $x_{0}$ não é um ponto fixo global da ação de $G$, o que implica que a ação de $G$ possui pontos fixos não globais, e assim pelo Lema 2.2.28, concluímos que $G$ é não-abeliano.

A ação $\Psi: G \times \mathbb{R} \rightarrow \mathbb{R}$, tem no máximo $N+2$ pontos fixos, logo pela Observação 2.2.27, $\Psi$ é fiel. Como $G$ é não-abeliano, então existem $g, f \in G$ com $g \circ f \circ g^{-1} \circ f^{-1} \neq I d$. Como a ação é fiel, segue que

$$
\Psi\left(g \circ f \circ g^{-1} \circ f^{-1}\right) \neq I d \Rightarrow \Psi(g) \circ \Psi(f) \circ \Psi(g)^{-1} \circ \Psi(f)^{-1} \neq I d
$$

e portanto, $\Psi(G)$ é não abeliano.

Assim, concluindo a prova de todos os itens do teorema.

Vamos aplicar o Teorema 4.3.1 no seguinte exemplo:

\section{Exemplo 4.3.2 Sejam:}

- G o grupo gerado por: $g(x)=2 x$ e $f(x)=x+1$,

- o ponto $x_{0}=0$,

- um homeomorfismo crescente do intervalo $q:(0,1) \rightarrow(0,1)$ com $N$ pontos fixos.

- a ação $\phi: \mathbb{Z} \rightarrow$ Homeo $_{+}([0,1])$ definida por $\phi(k)(x)=q^{k}(x)$.

Uma ação $\Psi(G)$, obtida a partir de $G$ abrindo a órbita do ponto $x_{0} e$ incluindo $\phi$ no intervalo, é não-abeliana, tem no máximo $N+2$ pontos fixos e possui um elemento com exatamente $N+2$ pontos fixos.

Prova. O grupo $G$ é um grupo de transformações afins e, portanto, ele possui no máximo um ponto fixo. Além disso, podemos descrever o grupo como:

$$
G=\left\{x \mapsto 2^{k} x+\frac{j}{2^{m}} \mid k \in \mathbb{Z}, j \in \mathbb{Z}, m \in \mathbb{Z}\right\} .
$$

Assim, a órbita do ponto 0 é dada por

$$
G .0=\left\{2^{k} 0+\frac{j}{2^{m}} \mid k \in \mathbb{Z}, j \in \mathbb{Z}, m \in \mathbb{Z}\right\}=\left\{\frac{j}{2^{m}} \mid j \in \mathbb{Z}, m \in \mathbb{Z}\right\}=\mathcal{D},
$$

o conjunto dos pontos diádicos. 
O estabilizador $H$ do ponto 0 é o subgrupo das aplicações com $j=0$,

$$
H=\left\{x \mapsto 2^{k} x \mid k \in \mathbb{Z}\right\} \simeq \mathbb{Z}
$$

Portanto, $(H$, o $)$ é isomorfo ao grupo $(\mathbb{Z},+)$.

Observe que a ação $\phi: \mathbb{Z} \rightarrow$ Homeo $_{+}([0,1])$ definida por $\phi(k)(x)=q^{k}(x)$ tem exatamente $N$ pontos fixos em $(0,1)$ (os mesmo pontos fixos de $q$ ).

Aplicando o Teorema 4.3.1, segue que $\Psi(G)$ tem no máximo $N+2$ pontos fixos, e como $H$ é não-trivial, existe elemento com $N+2$ pontos fixos. E como $f \in G$, com $f(0)=1$, temos que o ponto 0 não é ponto fixo global para ação de $G$, e assim, segue que $\Psi(G)$ é não-abeliana.

Definição 4.3.3 Uma ação de grupos $\theta: G \times X \rightarrow X$ é dita transitiva, se existe $x \in X$ cuja órbita é densa em $X$, ou seja, $\overline{G . x}=X$.

Corolário 4.3.4 Nas hipóteses do Teorema 4.3.1, se $\Psi(G)$ é ação de grupos transitiva então a ação $\phi$, incluída no intervalo, é livre de pontos fixos (caso $N=0)$.

Prova. Seja $h^{-1}\left(x_{0}\right)=[\alpha, \beta]$. Por hipótese, existe $x \in \mathbb{R}$ com $\overline{\Psi(G) \cdot x}=\mathbb{R}$. Logo,

$$
\Psi(G) . x \cap(\alpha, \beta) \neq \emptyset .
$$

E assim, escolha $y \in \Psi(G) . x \cap(\alpha, \beta)$, temos: $\Psi(G) . x=\Psi(G) . y$. Portanto, pela densidade, segue que

$$
(\alpha, \beta) \subset \overline{\Psi(G) \cdot y}
$$

Porém, $\Psi(g)(y) \in(\alpha, \beta)$ se e só se, $g\left(x_{0}\right)=x_{0}$. Em outras palavras, $g \in H$ (o estabilizador de $x_{0}$ ).

Logo, concluímos que: $(\alpha, \beta) \subset \overline{\Psi(H) . y}$, ou seja, $y$ tem órbita densa em $(\alpha, \beta)$. Porém, a ação $\Psi: H \rightarrow(\alpha, \beta)$ é conjugada a $\phi: H \rightarrow(0,1)$, que portanto, também possui órbita densa. Mas, todos os pontos fixos de $\phi(H)$ são globais, o que contradiz o fato de $\phi: H \rightarrow(0,1)$ possuir uma órbita densa, pois pontos fixos globais limitam todas as órbitas superiormente ou inferiormente. Portanto, $\phi: H \rightarrow(0,1)$ deve ser livre de pontos fixos, ou seja $N=0$.

Agora vamos apresentar um exemplo da aplicação do Teorema 4.3.1, que fornece uma ação de grupos transitiva.

\section{Exemplo 4.3.5 Sejam:}

- G o grupo das transformações afins racionais crescentes,

- o ponto $x_{0}=0$,

- a ação $\phi: \mathbb{Q}_{+} \rightarrow$ Homeo $_{+}([0,1])$ definida por $\phi(r)(x)=x^{r}$. 
Uma ação $\Psi(G)$ obtida a partir de $G$ abrindo a órbita do ponto $x_{0} e$ incluindo $\phi$ no intervalo, tem no máximo dois pontos fixos, é não-abeliana e possui uma órbita densa.

Prova. O grupo $G$ das transformações afins racionais pode ser descrito como

$$
G=\left\{x \mapsto a \cdot x+b \mid a \in \mathbb{Q}_{+}, b \in \mathbb{Q}\right\}
$$

A órbita do ponto 0 é dada por

$$
G .0=\left\{a .0+b \mid a \in \mathbb{Q}_{+}, b \in \mathbb{Q}\right\}=\mathbb{Q}
$$

e o estabilizador $H$ do ponto 0 é o subgrupo das aplicações com $b=0$.

$$
H=\left\{x \mapsto a . x \mid a \in \mathbb{Q}_{+}\right\} \simeq \mathbb{Q}_{+} .
$$

Portanto, $(H, \circ)$ é isomorfo ao grupo $\left(\mathbb{Q}_{+}, \times\right)$.

Observe que a ação $\phi: \mathbb{Q}_{+} \rightarrow$ Homeo $_{+}([0,1])$ definida por $\phi(r)(x)=x^{r}$ é livre de pontos fixos em $(0,1)$. Com efeito, existe $x \in(0,1) \operatorname{com} x^{r}=x$ só se $r=1$, e nesse caso estamos tomando a identidade do grupo.

Aplicando o Teorema 4.3.1, segue que $\Psi(G)$ tem no máximo 2 pontos fixos, e como $H$ é não-trivial, pelo terceiro item do teorema, segue que existe elemento com dois pontos fixos. E como $(x \mapsto x+1) \in G$, temos que o ponto 0 não é ponto fixo global para a ação de $G$, e assim, segue que $\Psi(G)$ é nãoabeliana.

Para concluir o exemplo, falta apenas a seguinte afirmação:

Afirmação. A ação de $\Psi(G)$ é transitiva.

Prova. Note que a ação $\phi: \mathbb{Q}_{+} \rightarrow H$ omeo $o_{+}([0,1])$ definida por $\phi(r)(x)=x^{r}$ é transitiva em $[0,1]$. Por exemplo, a órbita do ponto $\frac{1}{2}$ é densa. Com efeito:

$$
\phi(H) \cdot \frac{1}{2}=\left\{\left(\frac{1}{2}\right)^{r} \mid r \in \mathbb{Q}_{+}\right\}
$$

Tome $y \in(0,1)$ qualquer. Sabemos que: $\left(\frac{1}{2}\right)^{r^{\prime}}=y$, se $r^{\prime}=\log _{\frac{1}{2}} y \in \mathbb{R}_{+}$.

Escolha portanto, uma sequência de racionais positivos $\left\{r_{n}\right\}_{n \in \mathbb{N}}$, com $r_{n} \rightarrow r^{\prime}$, quando $n \rightarrow \infty$. E assim, temos $y \in \overline{\phi(H)}$. Como $y$ foi escolhido de forma arbitrária, temos que a órbita de $\frac{1}{2}$ é densa em $[0,1]$. E como, a ação de $\phi$ é incluída no intervalo, temos que a ação de $\Psi(G)$ é transitiva nos intervalos abertos, cuja união é dada por $h^{-1}(G .0)$ (onde $h$ é a semi-conjugação entre $\Psi(G)$ e $G)$.

Além disso, a órbita de 0 para a ação de $G$ também é densa em $\mathbb{R}$, pois $G .0=\mathbb{Q}$. Isso implica que $h^{-1}(G .0)$ é denso em $\mathbb{R}$. Portanto, $\Psi(G)$ possui 
órbita densa em $h^{-1}(G .0)$, que é denso em $\mathbb{R}$. Concluímos que $\Psi(G)$ possui uma órbita densa em $\mathbb{R}$.

Portanto, a demonstração do exemplo está completa.

Definição 4.3.6 Uma ação de grupos $\theta: G \times X \rightarrow X$ é dita minimal, se a órbita de todo ponto $x \in X$ é densa no conjuto $X$, ou seja, $\overline{G . x}=X$ para todo $x \in X$.

Corolário 4.3.7 Nas hipóteses do Teorema 4.3.1, $\Psi(G)$ não é minimal.

Prova. Por hipótese G. $x_{0}$ é enumerável e portanto, existe $y \in \mathbb{R}$, com $y \notin G . x_{0}$. Assim, a órbita de $y$ não contém $x_{0}, x_{0} \notin G$.y. E portanto, a órbita pela ação $\Psi(G)$ de $y$ não intersecta o intervalo compacto dado por $h^{-1}\left(x_{0}\right)$, concluíndo que $y$ não tem órbita densa e portanto $\Psi(G)$ não é minimal.

Observação 4.3.8 Como consequência dos Corolários 4.3.4 e 4.3.7, e do Exemplo 4.3.5 temos o seguinte cenário. Seja $\theta$ um grupo de homeomorfismos crescente de $\mathbb{R}$ com até $N \geq 2$ pontos fixos construido a partir do Teorema 4.3.1. Então

- se $N \geq 3$ : a ação não pode ser transitiva, e nem pode ser minimal,

- se $N=2$ : a ação pode ser transitiva, mas não pode ser minimal,

Além desses exemplos, temos os exemplos clássicos para $N \leq 1$, o grupo das translações e das aplicações afins. E portanto, segue que:

- se $N \leq 1$ : a ação pode ser transitiva, e pode ser minimal. 


\section{5}

\section{Ações com no máximo dois pontos fixos}

Este é o principal capítulo do trabalho. Em todo o texto, estivemos interessados nos grupos de homeomorfismos crescentes de $\mathbb{R}$ com no máximo $N$ pontos fixos. Já passamos pelos Teoremas de Hölder e de Solodov, que classificaram os grupos nos casos $N \leq 1$. Depois apresentamos a Abertura de Órbita, e com ela, desenvolvemos uma família de exemplos para os casos $N \geq 2$. Agora, vamos apresentar um resultado original que classifica, a menos de semi-conjugação, o caso $N=2$.

A prova da classificação será feita em várias etapas, que vão depender de caracteristicas dinâmicas da ação de grupos, como existência de pontos fixos, seus conjuntos minimais e seu comportamento assintótico.

\section{1}

\section{Caso existe um ponto fixo global}

Um ponto fixo global divide a reta em duas componentes invariantes, e nesse caso podemos aplicar o Teorema de Solodov em cada componente. É claro que um elemento que tem ponto fixo em uma das componentes, não pode ter na outra, se não violaria o máximo de dois pontos fixos contando com o global.

Desta forma o Teorema de Solodov implicará que uma das componentes deve ser livre de pontos fixos. Assim, basta aplicar o Teorema de Hölder nessa componente e concluir que o grupo é abeliano, e como consequência todos os pontos fixos são globais.

Segue o teorema desta subseção:

Teorema 5.1.1 Seja $G \subset \mathrm{Homeo}_{+}(\mathbb{R})$ com no máximo dois pontos fixos.

Se existe ponto fixo global então $G$ é abeliano.

Prova. Seja $x_{0} \in \mathbb{R}$ um ponto fixo global de $G$. Suponha que existam $g_{-}, g_{+}$ elementos não-triviais de $G$, tais que $g_{-}\left(y_{-}\right)=y_{-}$e $g_{+}\left(y_{+}\right)=y_{+}$, com $y_{-}<x_{0}<y_{+}$. Note que $\operatorname{Fix}\left(g_{+}\right)=\left\{x_{0}, y_{+}\right\}$e $\operatorname{Fix}\left(g_{-}\right)=\left\{y_{-}, x_{0}\right\}$, portanto $g_{+}$ não possui pontos fixos em $\left(-\infty, x_{0}\right)$ e $g_{-}$não possui pontos fixos em $\left(x_{0},+\infty\right)$.

Afirmação. O elemento $g_{+} \circ g_{-}^{-1}$ tem pelo menos 3 pontos fixos. 
Prova. Fixe $t:\left(x_{0},+\infty\right) \rightarrow \mathbb{R}$ homeomorfismo crescente, portanto a ação de $G$ restrita à $\left(x_{0},+\infty\right)$ tem no máximo um ponto fixo, e ela induz uma ação de $G$ em $\mathbb{R}$, onde $g \mapsto \bar{g}=t \circ g \circ t^{-1}$ também com no máximo um ponto fixo.

Porém, pelo Teorema de Solodov, existe $h: \mathbb{R} \rightarrow \mathbb{R}$ crescente, semiconjugação entre $G$ e um subgrupo de aplicações afins. Veja o diagrama abaixo:

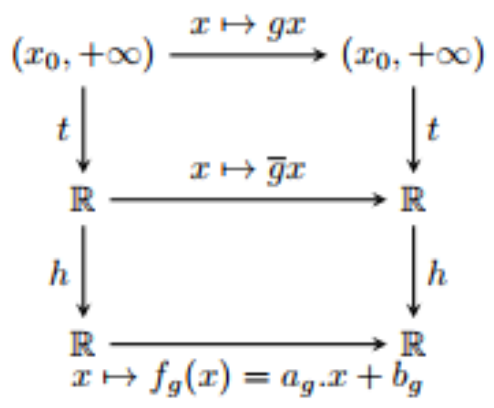

Como $g_{-}$não tem pontos fixos em $\left(x_{0},+\infty\right), \bar{g}_{-}$não tem pontos fixos em $\mathbb{R}$ e temos que $a_{g_{-}}=1$. E como $g_{+}$tem um ponto fixo em $\left(x_{0},+\infty\right), \bar{g}_{+}$tem um ponto fixo em $\mathbb{R}$ e temos que $a_{g_{+}} \neq 1$. Portanto,

$$
f_{g_{+}} \circ f_{g_{-}}^{-1}(x)=a_{g_{+}} \cdot\left(x-b_{g_{-}}\right)+b_{g_{+}} .
$$

Assim, $a_{g_{+} \circ g_{-}^{-1}}=a_{g_{+}} \neq 1$. Isto implica que $g_{+} \circ g_{-}^{-1}$ tem pontos fixos em $\left(x_{0},+\infty\right)$.

De forma análoga, podemos aplicar o Teorema de Solodov a componente $\left(-\infty, x_{0}\right)$ e concluir que $g_{+} \circ g_{-}^{-1}$ também tem pontos fixos nessa componente. $\mathrm{E}$ como $x_{0}$ é ponto fixo global, temos que $g_{+} \circ g_{-}^{-1}$ tem pontos fixos em $\left(-\infty, x_{0}\right)$, em $\left(x_{0},+\infty\right)$ e $x_{0}$. Portanto, tem no mínimo três pontos fixos.

Afirmação. Em pelo menos uma das componentes $\left(-\infty, x_{0}\right)$ e $\left(x_{0},+\infty\right)$ a ação de $G$ é livre de pontos fixos.

Prova. Como o elemento $g_{+} \circ g_{-}^{-1} \in G$ da afirmação anterior tem mais do que dois pontos fixos, segue que $g_{+} \circ g_{-}^{-1}=I d$, logo $g_{+}=g_{-}$que possui pelo menos três pontos fixos $\left\{y_{-}, x_{0}, y_{+}\right\}$e portanto $g_{+}=I d$. Absurdo, pois $g_{+}$é um elemento não-trivial.

Logo, não existem $g_{-}, g_{+} \in G$ não-triviais com pontos fixos em $\left(-\infty, x_{0}\right)$ e em $\left(x_{0},+\infty\right)$, respectivamente. Portanto, $G$ age livre de pontos fixos em $\left(-\infty, x_{0}\right)$ ou em $\left(x_{0},+\infty\right)$.

Corolário 5.1.2 Seja $G \subset \mathrm{Homeo}_{+}(\mathbb{R})$ com no máximo dois pontos fixos e um ponto fixo global então $G$ é abeliano.

Prova. Basta aplicar o Teorema de Hölder na componente em que $G$ age livre de pontos fixos. Segue daí que o grupo $G$ é abeliano. 
Concluindo a prova do Teorema 5.1.1.

\section{2}

\section{Caso sem pontos fixos globais}

Vamos enunciar o teorema principal e observar que, nas hipóteses do teorema, a ação sempre possui um conjunto minimal. Assim, separaremos a prova em casos dependendo dos tipos de conjuntos minimais que a ação possui. Cada caso será demonstrado em uma subseção distinta. Vamos também, adiar a demonstração da Proposição 5.2.4, dedicaremos mais adiante uma seção inteira a sua prova.

Segue então, o principal resultado de todo o trabalho:

Teorema 5.2.1 Seja $G \subset$ Homeo $_{+}(\mathbb{R})$ subgrupo com no máximo dois pontos fixos. Então um dos itens abaixo ocorre:

- Todos os pontos fixos de G são globais. Nesse caso, $G$ é abeliano.

- Existe um aplicação crescente e continua $\phi: \mathbb{R} \rightarrow \mathbb{R}$ e duas aplicações $a, b: G \rightarrow \mathbb{R}$, tais que para todo $g \in G$ e todo $x \in \mathbb{R}$

$$
\phi \circ g(x)=a(g) \cdot \phi(x)+b(g)
$$

Isto é, o diagrama abaixo é comutativo.

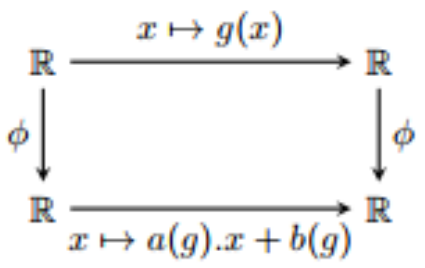

Prova. Caso $G$ possui um ponto fixo global, o resultado segue diretamente do Teorema 5.1.1. Vamos então considerar a ação de $G$ sem pontos fixos globais.

Afirmação. O grupo $G$ possui um conjunto minimal.

Prova. Tome $g \in G$ não-trivial, $g$ possui no máximo dois pontos fixos $\left\{x_{1}, x_{2}\right\}$. Como não existem pontos fixos globais, existem $g_{1}, g_{2} \in G$ tais que $g_{1}\left(x_{1}\right) \neq x_{1}$ e $g_{2}\left(x_{2}\right) \neq x_{2}$. Assim $g, g_{1}, g_{2}$ formam uma família sem um ponto fixo em comum. Pelo Corolário 2.3.6, segue que a aderência de toda órbita contém um conjunto minimal.

Como não existe ponto fixo global, pelo Teorema 2.3.4, existem três opções para os conjuntos minimais da reta. São elas,

(1) Todas as órbitas são densas em $\mathbb{R}$ e $\mathbb{R}$ é o único conjunto minimal. 
(2) Todos os conjuntos minimais são órbitas fechadas e bi-ilimitadas de $G$, que são conjuntos discretos. Além disso, existe $g \in G$ sem pontos fixos, tal que todos os conjuntos minimais são órbitas de $g$.

(3) Existe um único conjunto minimal de $G$ que é um conjunto do tipo Cantor bi-ilimitado, i.e., perfeito, bi-ilimitado e totalmente desconexo. Além disso, ele está contido na aderência de todas as órbitas.

Vamos agora provar cada um dos casos descritos pelos conjuntos minimais (na ordem (2), (1) e (3)).

\subsection{1}

\section{Prova do Teorema 5.2.1 no caso (2)}

Prova. A prova será conclusão do seguinte lema:

Lema 5.2.2 Se a ação de $G$ possui um conjunto minimal $K$ discreto biilimitado, então $G$ é livre de pontos fixos.

Prova. Suponha, por absurdo, que exista $g \in G$ com $\operatorname{Fix}(g) \neq \emptyset$. Seja $x_{m}$ o menor dos pontos fixos de $g$. Portanto, $g(y) \neq y$ para todo $y \in\left(-\infty, x_{m}\right)$, assim, ou $g(y)>y$ ou $g^{-1}(y)>y$, para todo $y \in\left(-\infty, x_{m}\right)$. Isso implica que ou $g^{n}(y) \rightarrow x_{m}$, ou $g^{-n}(y) \rightarrow x_{m}$. Logo, a órbita G.y se acumula em $x_{m}$, para todo $y \in\left(-\infty, x_{m}\right)$.

Como $K$ é bi-ilimitado, existe $x \in K \operatorname{com} x<x_{m}$. E como $K$ é invariante, $G . x \in K$. Logo, $K$ se acumula no ponto $x_{m}$ e portanto, não é um fechado discreto, o que contradiz a hipótese do lema.

Corolário 5.2.3 Seja $G \subset$ Homeo $_{+}(\mathbb{R})$ com no máximo dois pontos fixos, e $G$ possui um conjunto minimal discreto então $G$ é semi-conjugado à um subgrupo do grupo das translações.

Prova. Nessas hipóteses, pelo Lema 5.2.2 G age livre de pontos fixos. Portanto, basta aplicar o Teorema de Hölder.

Assim, concluímos a prova do teorema no caso (2) (nesse caso $a(g)=1$ para todo $g \in G)$. 


\section{2 .2}

\section{Prova do Teorema 5.2.1 no caso (1)}

Prova. Vamos apresentar uma proposição, cuja prova será adiada para a Seção 5.3 , e dela vamos concluir as demonstrações nos casos (1) e (3).

Proposição 5.2.4 Seja $g \in G$ não-trivial, com $\operatorname{Fix}(g)=\{a, b\}$. Então

$$
(G . a \cup G . b) \cap(a, b)=\emptyset .
$$

Prosseguindo com a prova do teorema no caso (1), temos que o resultado segue da seguinte afirmação.

Afirmação. Se o conjunto minimal de $G$ é $\mathbb{R}$, então os elementos não-triviais de $G$ tem no máximo um ponto fixo.

Prova. Suponha por absurdo, $g \in G \operatorname{com} \operatorname{Fix}(g)=\{a, b\}$. Pela Proposição 5.2.4, temos que:

$$
G . a \cap(a, b)=\emptyset \Rightarrow \overline{G . a} \cap(a, b)=\emptyset .
$$

Porém, $a \in \mathbb{R}$ pertence ao conjunto minimal, e portanto, pelo Lema 2.3.2. $\overline{G . a}=\mathbb{R}$. Absurdo.

Corolário 5.2.5 Se $G \subset \mathrm{Homeo}_{+}(\mathbb{R})$ com no máximo dois pontos fixos tal que $G$ tem $\mathbb{R}$ como conjunto minimal, então $G$ é semi-conjugada a um grupo de aplicações afins.

Prova. Nessas hipóteses, pela afirmação anterior, $G$ possui no máximo um ponto fixo, sem pontos fixos globais. Portanto, basta aplicar o Teorema de Solodov.

Assim, concluindo a prova do teorema no caso (1).

\subsection{3}

\section{Prova do Teorema 5.2.1 no caso (3)}

Começamos a prova desse caso com a seguinte afirmação.

Afirmação. Se o conjunto minimal de $G$ é do tipo Cantor bi-ilimitado, então todo ponto fixo pertence ao conjunto minimal.

Prova. Suponha por absurdo, que existem $g \in G$ não-trivial e $x \in \mathbb{R}$ tais que $g(x)=x$ e $x$ não pertence ao conjunto minimal $K$. Segue que $x \in K^{c}$ subconjunto aberto de $\mathbb{R}$. Seja então, $(a, b) \subset K^{c}$ a componente conexa de $K^{c}$ que contém $x$. 
Suponha primeiro que $g(a)>a$, como $g$ é crescente, temos

$$
a<g(a)<g(x)=x<b .
$$

E $a \in K$ implica que $g(a) \in K$, e assim $g(a) \in(a, b) \cap K$. Absurdo, pois $(a, b) \subset K^{c}$.

Caso $g(a)<a$, então $g^{-1}(a)>a$ o que implica no mesmo absurdo.

Portanto, $g(a)=a$ e de forma análoga, podemos concluir que $g(b)=b$. Temos $\operatorname{assim}\{a, x, b\} \in \operatorname{Fix}(g)$. Absurdo, pois $g \in G$ não-trivial.

Afirmação. Se $g \in G$ com $\operatorname{Fix}(g)=\{a, b\}$, então $(a, b) \cap K=\emptyset$.

Prova. Suponha, por absurdo, que existe $x \in(a, b) \cap K$. Pela afirmação anterior, sabemos que $a \in K$. E portanto, como $K$ é conjunto minimal, segue que $\overline{G \cdot a}=K$. Assim, temos

$$
x \in \overline{G \cdot a} \cap(a, b) \Rightarrow \overline{G \cdot a} \cap(a, b) \neq \emptyset \Rightarrow G \cdot a \cap(a, b) \neq \emptyset .
$$

Absurdo, pois pela Proposição 5.2.4, G.a $(a, b)=\emptyset$.

Lema 5.2.6 Se o minimal $K$ de $G$ é do tipo Cantor bi-ilimitado, então existem $h: \mathbb{R} \rightarrow \mathbb{R}$ crescente, contínua e sobrejetiva e $F \subset$ Homeo $_{+}(\mathbb{R})$ grupo isomorfo a $G$, agindo com no máximo um ponto fixo, satisfazendo para todo $g \in G$ e todo $x \in \mathbb{R}$

$$
h \circ g(x)=f_{g} \circ h(x) .
$$

Ou seja, o diagrama é comutativo

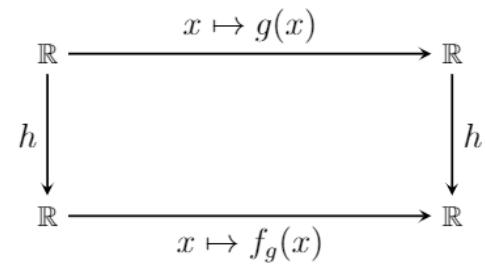

Prova. Note que $K \subset \mathbb{R}$ é um subconjunto fechado, ilimitado e sem pontos isolados. Então, tome $h: \mathbb{R} \rightarrow \mathbb{R}$, como no Lema 2.1.19, $h$ crescente, sobrejetiva e contínua. Note que $h$ é constante nas componentes do complementar $K^{c}$.

Pelo Lema 2.2.34, como $K$ é $G$-invariante, temos que $\phi: G \rightarrow$ $\mathrm{Homeo}_{+}(\mathbb{R})$ definido por

$$
\phi(g)(x):=h \circ g \circ h^{-1}(x)
$$

é homomorfismo de grupos. Portanto, $F:=\phi(G) \subset$ Homeo $_{+}(\mathbb{R})$ é grupo, bem definido. Além disso, $\phi: G \rightarrow F$ é injetiva, com efeito: 
Tome $g \in G$ não-trivial. Como cada elemento de $G$ tem no máximo dois pontos fixos, segue que $g(x)=x$ no máximo em dois pontos. Portanto, existe $y \in K \operatorname{com} g(y) \neq y$. Aplicando $\phi(g)$ no ponto $h(y)$ e lembrando que $h$ é bijetiva entre $K$ e $h(K)$, segue

$$
\phi(g)(h(y))=h \circ g \circ h^{-1}(h(y))=h \circ g(y) \neq h(y)
$$

Assim, temos $\phi(g) \neq I d$, e portanto, $\phi$ é injetiva. Concluímos também, como $F=\phi(G)$, que o grupo $F$ é isomorfo a $G$.

Para concluir que o grupo $F$ age com no máximo um ponto fixo, lembre-se que:

$$
\operatorname{Fix}(\phi(g))=h(\operatorname{Fix}(g)) .
$$

Portanto, caso \#Fix $(g) \leq 1$, então $\# \operatorname{Fix}(\phi(g)) \leq 1$. E caso $g \in G$ com dois pontos fixos, $\operatorname{Fix}(g)=\{a, b\}$. Temos que $(a, b)$ é componente do complementar $K^{c}$, e portanto $h(a)=h(b)$. Assim:

$$
\operatorname{Fix}(\phi(g))=h(\operatorname{Fix}(g))=h(\{a, b\})=\{h(a), h(b)\} .
$$

Como $h(a)=h(b)$, temos \# $\operatorname{Fix}(\phi(g))=1$. E portanto, o grupo $F$ age com no máximo um ponto fixo.

Corolário 5.2.7 Seja $G \subset$ Homeo $_{+}(\mathbb{R})$ com no máximo dois pontos fixos, e com um conjunto minimal do tipo Cantor bi-ilimitado, então $G$ é semiconjugado à um subgrupo do grupo afim.

Prova. Nessas hipóteses, a ação de $G$ é semi-conjugada a ação do grupo $F$, que age com no máximo um ponto fixo, e sem pontos fixos globais. Portanto, aplicando o Teorema de Solodov, $F$ é semi-conjugada à um grupo de aplicações afins. Assim, temos

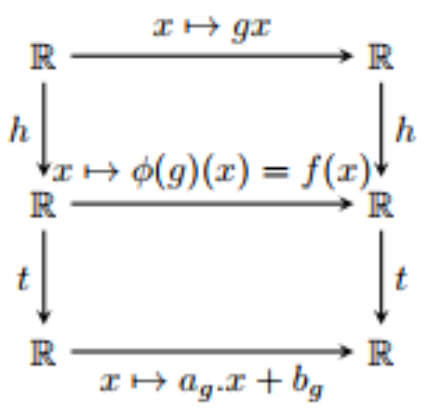

Ou seja, para todo $g \in G$ e para todo $x \in \mathbb{R}$

$$
t \circ h \circ g(x)=a_{g} . t \circ h(x)+b_{g}
$$


Portanto segue que $G$ é semi-conjugada à um grupo de aplicações afins.

Assim, concluímos o teorema no caso (3), e portanto concluímos a prova do Teorema 5.2.1, a menos da prova da Proposição 5.2.4.

\section{3}

\section{Prova da Proposição 5.2.4}

Nesse capítulo será feita a demonstração da Proposição 5.2.4. Assim, completaremos as demonstrações do Capítulo 5. Para a demonstração da proposição vamos dividir todos os homeomorfismos reais crescentes com dois pontos fixos pelo seu comportamento assintótico (veja Definição 5.3.3). E provaremos a Proposição 5.2.4 em cada um desses casos separadamente, porém, usaremos o mesmo modelo de demonstração para todos os casos.

Vamos começar com os seguintes lemas, que simplificarão bastante nossas demonstrações:

Lema 5.3.1 Seja $G \subset \mathrm{Homeo}_{+}(\mathbb{R})$ subgrupo com no máximo dois pontos fixos. Se para todo $g \in G$ com exatamente dois pontos fixos temos que

$$
G . a_{g} \cap\left(a_{g}, b_{g}\right)=\emptyset \text {, onde } \operatorname{Fix}(g)=\left\{a_{g}, b_{g}\right\} \text {, }
$$

então para todo $f \in G$ com exatamente dois pontos fixos,

$$
\left(G . a_{f} \cup G . b_{f}\right) \cap\left(a_{f}, b_{f}\right)=\emptyset .
$$

(Ou seja, basta olhar uma das órbitas na Proposição 5.2.4).

Prova. Suponha por absurdo que existe $f \in G \operatorname{com} \operatorname{Fix}(f)=\left\{a_{f}, b_{f}\right\}$ e

$$
\left(G . a_{f} \cup G . b_{f}\right) \cap\left(a_{f}, b_{f}\right) \neq \emptyset
$$

ou seja, existe $h \in G \operatorname{com} h\left(b_{f}\right) \in\left(a_{f}, b_{f}\right)$.

Pela hipótese temos $h\left(a_{f}\right) \notin\left(a_{f}, b_{f}\right)$, mas $h\left(a_{f}\right)<h\left(b_{f}\right)<b_{f}$ pois $h$ é crescente. E portanto,

$$
h\left(a_{f}\right)<a_{f}<h\left(b_{f}\right)<b_{f} .
$$

Note que, $a_{f} \in\left(h\left(a_{f}\right), h\left(b_{f}\right)\right)$ e $a_{f} \in G . h\left(a_{f}\right)$, pois $a_{f}=h^{-1}\left(h\left(a_{f}\right)\right)$. Segue então

$$
G . h\left(a_{f}\right) \cap\left(h\left(a_{f}\right), h\left(b_{f}\right)\right) \neq \emptyset .
$$


Agora considere $f^{\prime}:=h \circ f \circ h^{-1}$ e observe que $f^{\prime} \in G$ e $\operatorname{Fix}\left(f^{\prime}\right)=$ $\left\{h\left(a_{f}\right), h\left(b_{f}\right)\right\}=\left\{a_{f^{\prime}}, b_{f^{\prime}}\right\}$. Temos então

$$
G \cdot a_{f^{\prime}} \cap\left(a_{f^{\prime}}, b_{f^{\prime}}\right) \neq \emptyset
$$

Absurdo, pois contradiz nossa hipótese.

Lema 5.3.2 Sejam $G \subset \mathrm{Homeo}_{+}(\mathbb{R})$ subgrupo com no máximo dois pontos fixos e $g \in G$ não-trivial, com $\operatorname{Fix}(g)=\{a, b\}$. Se $h \in G$ é tal que $h(a) \neq a$, então $h(b) \neq b$.

Prova. Tome $h \in G$ com $h(a) \neq a$. Suponha por absurdo, que $h(b)=b$ (como $h$ é bijeção, $h(a) \neq h(b)=b$ ). Construa o subgrupo $H$ gerado por $h, g$. $H=\langle h, g\rangle \subset G$. Então $H$ tem no máximo dois pontos fixos. Porém, para todo $f \in H, f(b)=b$. Portanto, $H$ tem no máximo dois pontos fixos e possui um ponto fixo global. Pelo Teorema 5.1.1, $H$ é abeliano e portanto,

$$
g \circ h=h \circ g \Rightarrow g(h(a))=h(g(a))=h(a) .
$$

Ou seja, $g(h(a))=h(a)$. Absurdo, pois como $h(a) \neq a$ e $h(a) \neq b$, temos que $\operatorname{Fix}(g)$ contém pelo menos três pontos $\{a, h(a), b\}$, o que é impossível para $g \in G$ não-trivial.

Para prosseguir na demonstração da proposição, precisamos da seguinte definição:

Definição 5.3.3 Seja $g \in \mathrm{Homeo}_{+}(\mathbb{R})$, com exatamente dois pontos fixos $\{a, b\}$ e $a<b$. Dizemos que g é do tipo $\left(\delta_{0}, \delta_{1}, \delta_{2}\right)$, com $\delta_{i} \in\{-,+\}$, se:

- $\delta_{0}$ é sinal de $g(x)-x$, para todo $x \in(-\infty, a)$,

- $\delta_{1}$ é sinal de $g(x)-x$, para todo $x \in(a, b)$,

- $\delta_{2}$ é sinal de $g(x)-x$, para todo $x \in(b,+\infty)$.

Observação 5.3.4 $O$ sinal de $g(x)-x$ está bem definido para todo $x \in$ $\mathbb{R} \backslash\{a, b\}$, pois $\{a, b\}$ são os únicos zeros de $g(x)-x$. E pela continuidade de $g(x)-x$, o sinal deve ser constante em cada uma das componentes conexas $(-\infty, a),(a, b),(b,+\infty)$. E assim, a definição é coerente.

A partir de agora, vamos sempre considerar $g \in \mathrm{Homeo}_{+}(\mathbb{R})$ com exatamente dois pontos fixos $\{a, b\}$, onde $a<b$.

Nos Lemas 5.3.5, 5.3.6 e 5.3.7, veremos como os sinais da Definição 5.3.3 se comportam diante da inversão, potenciação e conjugação. 
Lema 5.3.5 Se $g$ do tipo $\left(\delta_{0}, \delta_{1}, \delta_{2}\right)$, então $g^{-1}$ é do tipo $\left(\delta_{0}^{\prime}, \delta_{1}^{\prime}, \delta_{2}^{\prime}\right)$, onde $\delta_{i} \neq \delta_{i}^{\prime}$. Ou seja, g e $g^{-1}$ têm todos os sinais trocados.

Prova. Seja $g \in \mathrm{Homeo}_{+}(\mathbb{R})$, com exatamente dois pontos fixos $\{a, b\}$ e $a<b$. Note que $g(x)$ e $x$ estão na mesma componente conexa de $\mathbb{R} \backslash\{a, b\}$. Caso contrário, haveria um ponto fixo entre $x$ e $g(x)$, o que é um absurdo, pois $g$ é crescente. Por fim, basta notar que:

$$
g(x)-x \text { tem o sinal contrário a } x-g(x)=g^{-1}(g(x))-g(x) .
$$

Portanto, $g(x)-x$ e $g^{-1}(x)-x$ tem sinais trocados em cada componente.

Lema 5.3.6 Seja g do tipo $\left(\delta_{0}, \delta_{1}, \delta_{2}\right)$, então, para todo $n$ inteiro positivo, $g^{n}$ também é do tipo $\left(\delta_{0}, \delta_{1}, \delta_{2}\right)$.

Prova. Note que $\operatorname{Fix}\left(g^{k}\right)=\operatorname{Fix}(g)=\{a, b\}$, para todo $k \in\{1, \ldots, n\}$. E além disso, $x, g(x), g^{2}(x), \ldots, g^{n}(x)$ estão na mesma componente conexa de $\mathbb{R} \backslash\{a, b\}$. Portanto,

$g(x)-x$ tem o mesmo sinal de $g\left(g^{k}(x)\right)-g^{k}(x)$ para todo $k \in\{1, \ldots n\}$.

Assim, o sinal se mantém após somar todos

$$
g(x)-x \text { tem o mesmo sinal de } \sum_{k=0}^{n-1} g\left(g^{k}(x)\right)-g^{k}(x)=g^{n}(x)-x
$$

Portanto, $g(x)-x$ e $g^{n}(x)-x$ tem os mesmos sinais em cada componente, e assim $g$ e $g^{n}$ são do mesmo tipo.

Lema 5.3.7 Seja g do tipo $\left(\delta_{0}, \delta_{1}, \delta_{2}\right)$, então, para todo $h \in \mathrm{Homeo}_{+}(\mathbb{R})$, $h \circ g \circ h^{1}$ também é do tipo $\left(\delta_{0}, \delta_{1}, \delta_{2}\right)$.

Prova. Note que $\operatorname{Fix}\left(h \circ g \circ h^{-1}\right)=h(\operatorname{Fix}(g))=\{h(a), h(b)\}$. E como $h$ é crescente, $x$ e $h(x)$ estão em componentes correspondentes de $\mathbb{R} \backslash\{a, b\}$ e $\mathbb{R} \backslash\{h(a), h(b)\}$. (i.e. se $x \in(-\infty, a)$ então $h(x) \in(-\infty, h(a))$, respectivamente para as outras componentes). E como $h$ é crescente, sinal de $\alpha-\beta$ é igual ao sinal de $h(\alpha)-h(\beta)$, para todos $\alpha, \beta \in \mathbb{R}$. Portanto,

$$
g(x)-x \text { tem o mesmo sinal de } h(g(x))-h(x)=h \circ g \circ h^{-1}(h(x))-h(x)
$$

Portanto, $g(x)-x$ e $h \circ g \circ h^{-1}(x)-x$ têm os mesmos sinais em cada componente correspondente, $\log g$ e $h \circ g \circ h^{-1}$ são do mesmo tipo. 
Observação 5.3.8 A definição 5.3.3 separa os homeomorfismos com exatamente dois pontos fixos, em oito tipos:

$$
(+,+,+),(+,+,-),(+,-,+),(+,-,-),(-,+,+),(-,+,-),(-,-,+) e(-,-,-) .
$$

O lema seguinte permite reduzir o estudo a apenas quatro casos.

Lema 5.3.9 Se a Proposição 5.2.4 é verdadeira para todo $g \in G$ do tipo $\left(\delta_{0}, \delta_{1}, \delta_{2}\right)$, então também é verdadeira para todo $f \in G$ do tipo $\left(\delta_{0}^{\prime}, \delta_{1}^{\prime}, \delta_{2}^{\prime}\right)$, onde $\delta_{i} \neq \delta_{i}^{\prime}$, ou seja, $f$ com todos os sinais trocados.

Prova. Tome $f \in G$, do tipo $\left(\delta_{0}^{\prime}, \delta_{1}^{\prime}, \delta_{2}^{\prime}\right)$ com $\operatorname{Fix}(f)=\{x, y\}$. Note que, pelo Lema 5.3.5, $f^{-1}$ é do tipo $\left(\delta_{0}, \delta_{1}, \delta_{2}\right)$, e $\operatorname{Fix}\left(f^{-1}\right)=\operatorname{Fix}(f)=\{x, y\}$. Portanto, aplicando a Proposição 5.2.4 em $f^{-1}$, temos que

$$
(G . x \cup G . y) \cap(x, y)=\emptyset .
$$

Assim, $f$ também satisfaz a Proposição 5.2.4. E como $f \in G$ do tipo $\left(\delta_{0}^{\prime}, \delta_{1}^{\prime}, \delta_{2}^{\prime}\right)$ foi escolhido de forma arbitrária, temos que a proposição é válida para todo $f \in G$ do tipo $\left(\delta_{0}^{\prime}, \delta_{1}^{\prime}, \delta_{2}^{\prime}\right)$.

Como consequência do Lema 5.3.9, os casos $(+,+,+),(-,+,+)$, $(-,-,+)$ e $(+,-,+)$ abrangem todos os tipos de homeomorfismos necessários para a prova da Proposição 5.2.4. Os três primeiros serão chamados de casos de tangência, e serão demonstrados na subseção adiante.

\subsection{1}

\section{Prova da Proposição 5.2.4 nos casos de tangência}

Seja $g \in G$ do tipo $\left(\delta_{0}, \delta_{1}, \delta_{2}\right)$ com pontos fixos $\{a, b\}$. Suponha por absurdo que:

$$
G . a \cap(a, b) \neq \emptyset .
$$

Portanto, existe $h \in G$, tal que $h(a) \in(a, b)$ e pelo Lema 5.3.2, $h(b) \neq b$. Defina $\tilde{a}:=h(a)$ e $\tilde{b}:=h(b)$. Temos assim, duas opções: $\tilde{b}>b$ e $\tilde{b}<b$.

Mostraremos para cada um dos casos de tangência, que ambas opções $\tilde{b}>b$ e $\tilde{b}<b$, implicam na existência de um elemento $q \in G$ não=trivial, com pelo menos três pontos fixos. O que é um absurdo, pois $G$ por hipótese age com no máximo dois pontos fixos.

- Caso $(+,+,+), \operatorname{com} \tilde{b}>b$ :

Afirmação. Existe $q \in G$ tal que:

$$
q(a)<a<\tilde{a}<q(\tilde{a})<q(b)<b<\tilde{b}<q(\tilde{b}) .
$$


Portanto, $q$ tem pelo menos três pontos fixos.

Prova. Tome $f=h \circ g \circ h^{-1} \in G$. Note que $\operatorname{Fix}(f)=\{h(a), h(b)\}=\{\tilde{a}, \tilde{b}\}$ e pelo Lema 5.3.5 $f$ também é do tipo $(+,+,+)$. E defina $q:=f^{-1} \circ g \in G$.

Como $g$ é do tipo $(+,+,+)$, temos que $g(x)>x$ para todo $x \neq a, b$. E como $f$ também é do tipo $(+,+,+)$, temos que $f(x)>x$ para todo $x \neq \tilde{a}, \tilde{b}$. Temos a seguinte figura, que esboça a posição destes elementos:

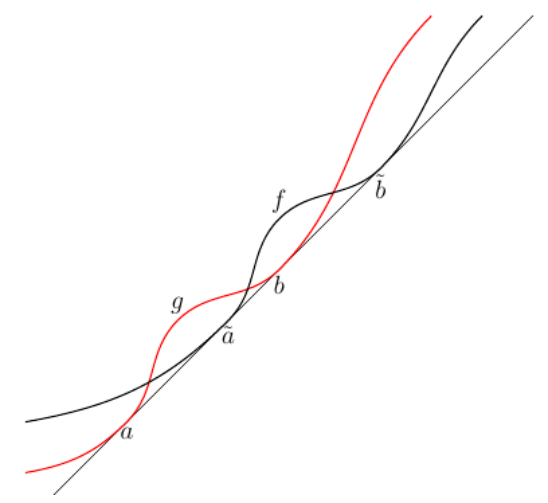

E de forma mais precisa, temos as seguintes desigualdades:

$$
\begin{gathered}
g(a)=a<f(a) \Rightarrow f^{-1} \circ g(a)<a \Rightarrow q(a)<a, \\
g(\tilde{a})>\tilde{a}=f(\tilde{a}) \Rightarrow f^{-1} \circ g(\tilde{a})>\tilde{a} \Rightarrow q(\tilde{a})>\tilde{a} \\
g(b)=b<f(b) \Rightarrow f^{-1} \circ g(b)<b \Rightarrow q(b)<b, \\
g(\tilde{b})>\tilde{b}=f(\tilde{b}) \Rightarrow f^{-1} \circ g(\tilde{b})>\tilde{b} \Rightarrow q(\tilde{b})>\tilde{b} .
\end{gathered}
$$

Por fim, lembre-se que $\tilde{a}=h(a) \in(a, b)$ e estamos no caso $\tilde{b}>b$. Portanto: $a<\tilde{a}<b<\tilde{b}$. Assim, usando o fato de que $q$ é crescente e as quatro desigualdades acima, fica claro que:

$$
q(a)<a<\tilde{a}<q(\tilde{a})<q(b)<b<\tilde{b}<q(\tilde{b}) .
$$

Além disso, o sinal de $q(x)-x$ troca pelo menos três vezes entre $a$ e $\tilde{b}$. Portanto, $q$ possui pelo menos três pontos fixos.

- Caso $(+,+,+)$, com $\tilde{b}<b$ :

Afirmação. Existe $q \in G$ e $y \in \mathbb{R}$, tais que:

$$
q(a)<a<\tilde{a}<q(\tilde{a})<q(b)<b<y<q(y) .
$$

Portanto, $q$ tem pelo menos três pontos fixos. 
Prova. Tome novamente $f=h \circ g \circ h^{-1} \in G$. Pelo Lema 5.3.6, $g^{n} \in G$ e é do tipo $(+,+,+)$ com pontos fixos $\{a, b\}$, para todo $n \in \mathbb{N}$. Além disso,

$$
\lim _{n \rightarrow+\infty} g^{n}(x)=+\infty, \text { para todo } x \in(b, \infty)
$$

Assim, fixando $y>b$, existe $n_{0} \in \mathbb{N}$, tal que: $g^{n_{0}}(y)>f(y)$. Defina então, $q:=f^{-1} \circ g^{n_{0}} \in G$.

Como $g^{n_{0}}$ é do tipo $(+,+,+)$, temos que $g^{n_{0}}(x)>x$ para todo $x \neq a, b$. E como $f$ também é do tipo $(+,+,+)$, temos que $f(x)>x$ para todo $x \neq \tilde{a}, \tilde{b}$. Segue uma figura que esboça a situação:

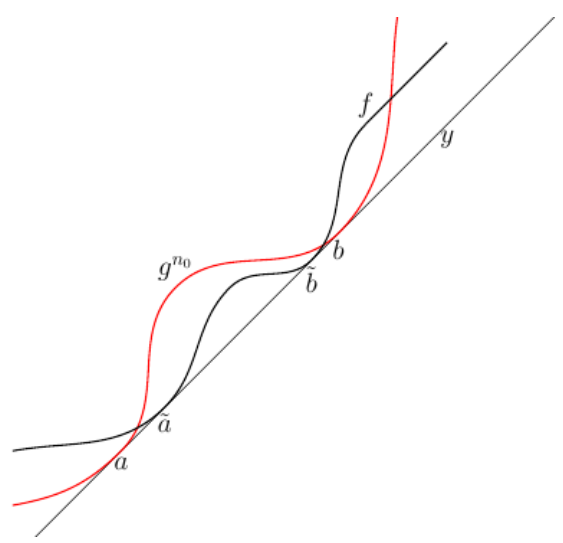

E de forma mais precisa, temos as seguintes desigualdades:

$$
\begin{gathered}
g^{n_{0}}(a)=a<f(a) \Rightarrow f^{-1} \circ g^{n_{0}}(a)<a \Rightarrow q(a)<a, \\
g^{n_{0}}(\tilde{a})>\tilde{a}=f(\tilde{a}) \Rightarrow f^{-1} \circ g^{n_{0}}(\tilde{a})>\tilde{a} \Rightarrow q(\tilde{a})>\tilde{a}, \\
g^{n_{0}}(b)=b<f(b) \Rightarrow f^{-1} \circ g^{n_{0}}(b)<b \Rightarrow q(b)<b, \\
g^{n_{0}}(y)>f(y) \Rightarrow f^{-1} \circ g^{n_{0}}(y)>y \Rightarrow q(y)>y .
\end{gathered}
$$

Por fim, lembre-se que $\tilde{a} \in(a, b)$ e que $y>b$. Portanto: $a<\tilde{a}<b<y$. Assim, usando o fato de que $q$ é crescente e as quatro desigualdades acima, fica claro que:

$$
q(a)<a<\tilde{a}<q(\tilde{a})<q(b)<b<y<q(y) .
$$

Além disso, o sinal de $q(x)-x$ troca pelo menos três vezes entre $a$ e $y$. Portanto, $q$ possui pelo menos três pontos fixos.

Concluímos assim a prova da Proposição 5.2.4 no caso $(+,+,+)$.

- Caso $(-,+,+), \operatorname{com} \tilde{b}>b$ : 
Afirmação. Existem $q \in G$ e $y \in \mathbb{R}$, tais que:

$$
\tilde{a}<q(\tilde{a})<q(b)<b<\tilde{b}<q(\tilde{b})<q(y)<y
$$

Portanto, $q$ possui pelo menos três pontos fixos.

Prova. Tome $f=h \circ g \circ h^{-1} \in G$. Note que usando os Lemas 5.3.6 e 5.3.7, temos $f^{n} \in G$ é do tipo $(-,+,+)$ e tem pontos fixos $\{h(a), h(b)\}=\{\tilde{a}, \tilde{b}$ para todo $n \in \mathbb{N}$. Além disso,

$$
\lim _{n \rightarrow+\infty} f^{n}(x)=+\infty \text {, para todo } x \in(\tilde{b},+\infty) .
$$

Assim, fixando $y>\tilde{b}$, existe $n_{0} \in \mathbb{N}$, tal que: $f^{n_{0}}(y)>g(y)$. Defina então, $q:=f^{-n_{0}} \circ g \in G$.

Como $g$ é do tipo $(-,+,+)$, temos que $g(x)>x$ para todo $x>a$ e $x \neq b$. E como $f^{n_{0}}$ também é do tipo $(-,+,+)$, temos que $f^{n_{0}}(x)>x$ para todo $x>\tilde{a}$ e $x \neq \tilde{b}$. Segue então, uma figura da situação:

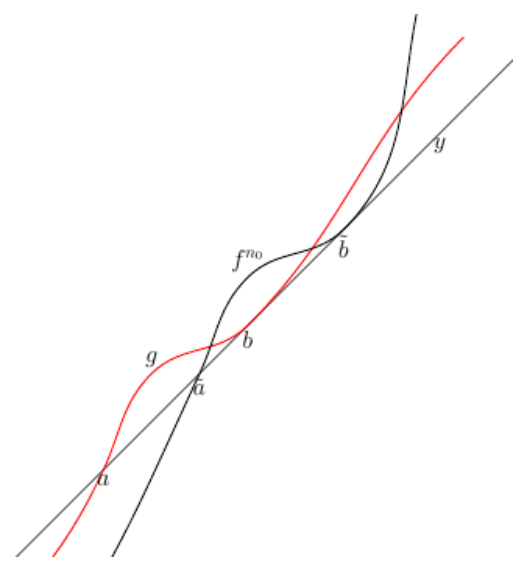

E mais precisamente, temos as seguintes desigualdades:

$$
\begin{gathered}
g(\tilde{a})>\tilde{a}=f^{n_{0}}(\tilde{a}) \Rightarrow f^{-n_{0}} \circ g(\tilde{a})>\tilde{a} \Rightarrow q(\tilde{a})>\tilde{a}, \\
g(b)=b<f^{n_{0}}(b) \Rightarrow f^{-n_{0}} \circ g(b)<b \Rightarrow q(b)<b, \\
g(\tilde{b})>\tilde{b}=f^{n_{0}}(\tilde{b}) \Rightarrow f^{-n_{0}} \circ g(\tilde{b})>\tilde{b} \Rightarrow q(\tilde{b})>\tilde{b}, \\
g(y)<f^{n_{0}}(y) \Rightarrow f^{-n_{0}} \circ g(y)<y \Rightarrow q(y)<y .
\end{gathered}
$$

Por fim, lembre-se que $\tilde{a} \in(a, b)$, que estamos no caso $b<\tilde{b}$ e que escolhemos $y>\tilde{b}$. Portanto: $\tilde{a}<b<\tilde{b}<y$. Assim, usando o fato de que $q$ é crescente e as quatro desigualdades acima, fica claro que:

$$
\tilde{a}<q(\tilde{a})<q(b)<b<\tilde{b}<q(\tilde{b})<q(y)<y
$$


Além disso, o sinal de $q(x)-x$ troca pelo menos três vezes entre $\tilde{a}$ e $y$. Portanto, $q$ possui pelo menos três pontos fixos.

- Caso $(-,+,+), \operatorname{com} \tilde{b}<b$ :

Afirmação. Existem $q \in G$ e $y_{1}, y_{2} \in \mathbb{R}$, tais que:

$$
q\left(y_{1}\right)<y_{1}<a<q(a)<q(b)<b<y_{2}<q\left(y_{2}\right) .
$$

Portanto, $q$ possui pelo menos três pontos fixos.

Prova. Tome novamente $f=h \circ g \circ h^{-1} \in G$. Note que pelo Lema 5.3.6, $g^{n} \in G$ é do tipo $(-,+,+)$ com pontos fixos $\{a, b\}$, para todo $n \in \mathbb{N}$. Além disso,

$$
\lim _{n \rightarrow+\infty} g^{n}(x)=-\infty \text {, para todo } x \in(-\infty, a) .
$$

Assim, fixando $y_{1}<a$, existe $n_{1} \in \mathbb{N}$, tal que: $g^{n}\left(y_{1}\right)<f\left(y_{1}\right)$, para todo $n \geq n_{1}$. E também temos que

$$
\lim _{n \rightarrow+\infty} g^{n}(x)=+\infty \text {, para todo } x \in(b, \infty)
$$

Assim, fixando $y_{2}>b$, existe $n_{2} \in \mathbb{N}$, tal que: $g^{n}\left(y_{2}\right)>f\left(y_{2}\right)$, para todo $n \geq n_{2}$. Defina então, $q:=f^{-1} \circ g^{n_{0}} \in G$, onde $n_{0}=\max \left\{n_{1}, n_{2}\right\}$.

Como $g^{n_{0}}$ é do tipo $(-,+,+)$, temos que $g^{n_{0}}(x)<x$ para todo $x<a$ e $g^{n_{0}}(x)>x$ para todo $x>b$. E como $f$ também é do tipo $(-,+,+)$, temos que $f(x)<x$ para todo $x<\tilde{a}$ e $f(x)>x$ para todo $x<\tilde{b}$. Em particular $f(a)<a$ e $f(b)>b$, pois $a<\tilde{a}<\tilde{b}<b$. Portanto, segue o esboço:

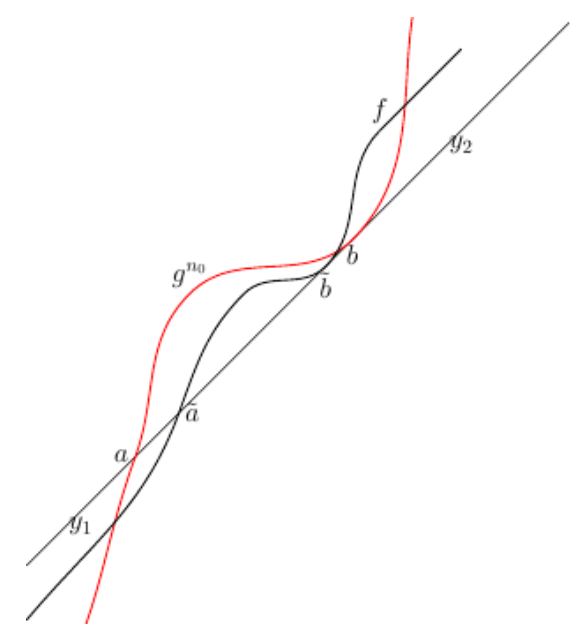

E de forma mais precisa, temos as seguintes desigualdades:

$$
\begin{gathered}
g^{n_{0}}\left(y_{1}\right)<f\left(y_{1}\right) \Rightarrow f^{-1} \circ g^{n_{0}}\left(y_{1}\right)<y_{1} \Rightarrow q\left(y_{1}\right)<y_{1}, \\
g^{n_{0}}(a)=a>f(a) \Rightarrow f^{-1} \circ g^{n_{0}}(a)>a \Rightarrow q(a)>a,
\end{gathered}
$$




$$
\begin{gathered}
g^{n_{0}}(b)=b<f(b) \Rightarrow f^{-1} \circ g^{n_{0}}(b)<b \Rightarrow q(b)<b, \\
g^{n_{0}}\left(y_{2}\right)>f\left(y_{2}\right) \Rightarrow f^{-1} \circ g^{n_{0}}\left(y_{2}\right)>y_{2} \Rightarrow q\left(y_{2}\right)>y_{2} .
\end{gathered}
$$

Por fim, lembre-se que $a<b$, e que escolhemos $y_{1}<a$ e $y_{2}>b$. Portanto: $y_{1}<a<b<y_{2}$. Assim, usando o fato de que $q$ é crescente e as quatro desigualdades acima, fica claro que:

$$
q\left(y_{1}\right)<y_{1}<a<q(a)<q(b)<b<y_{2}<q\left(y_{2}\right) .
$$

Além disso, o sinal de $q(x)-x$ troca pelo menos três vezes entre $y_{1}$ e $y_{2}$. Portanto, $q$ possui pelo menos três pontos fixos.

Concluímos assim a prova da Proposição 5.2.4 no caso $(-,+,+)$.

- Caso $(-,-,+), \operatorname{com} \tilde{b}>b$ :

Afirmação. Existem $q \in G$ e $y \in \mathbb{R}$, tais que:

$$
a<q(a)<q(\tilde{a})<\tilde{a}<b<q(b)<q(y)<y .
$$

Portanto, $q$ possui pelo menos três pontos fixos.

Prova. Tome $f=h \circ g \circ h^{-1} \in G$. Note que pelos Lemas 5.3.6 e 5.3.7 temos $f^{n} \in G$ é do tipo $(-,-,+)$ e tem pontos fixos $\{h(a), h(b)\}=\{\tilde{a}, \tilde{b}\}$ para todo $n \in \mathbb{N}$. Além disso,

$$
\lim _{n \rightarrow+\infty} f^{n}(x)=+\infty \text {, para todo } x \in(\tilde{b},+\infty) .
$$

Assim, fixando $y>\tilde{b}$, existe $n_{0} \in \mathbb{N}$, tal que: $f^{n_{0}}(y)>g(y)$. Defina então, $q:=f^{-n_{0}} \circ g \in G$.

Como $g$ é do tipo $(-,-,+)$, temos que $g(x)<x$ para todo $x<b$ e $x \neq a$. E como $f^{n_{0}}$ também é do tipo $(-,-,+)$, temos que $f^{n_{0}}(x)<x$ para todo $x<\tilde{b}$ e $x \neq \tilde{a}$. Esboço:

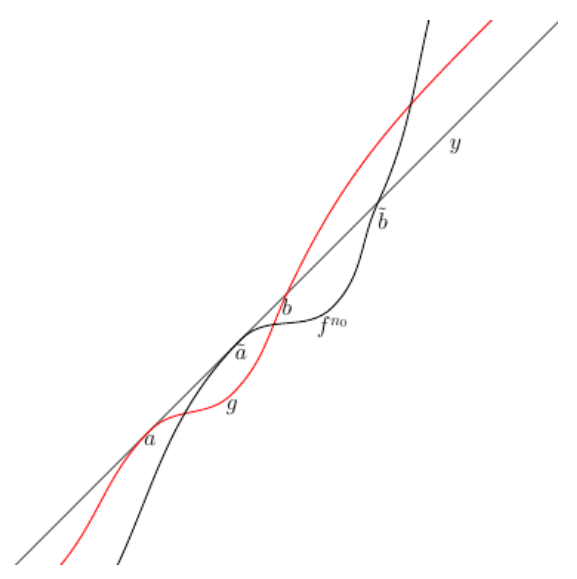


Mais precisamente, seguem as desigualdades:

$$
\begin{gathered}
g(a)=a>f^{n_{0}}(a) \Rightarrow f^{-n_{0}} \circ g(a)>a \Rightarrow q(a)>a, \\
g(\tilde{a})<\tilde{a}=f^{n_{0}}(\tilde{a}) \Rightarrow f^{-n_{0}} \circ g(\tilde{a})<\tilde{a} \Rightarrow q(\tilde{a})<\tilde{a}, \\
g(b)=b>f^{n_{0}}(b) \Rightarrow f^{-n_{0}} \circ g(b)>b \Rightarrow q(b)>b, \\
g(y)<f^{n_{0}}(y) \Rightarrow f^{-n_{0}} \circ g(y)<y \Rightarrow q(y)<y .
\end{gathered}
$$

Por fim, lembre-se que $\tilde{a} \in(a, b)$, e que escolhemos $y>b$. Portanto: $a<\tilde{a}<b<y$. Assim, usando o fato de que $q$ é crescente e as quatro desigualdades acima, fica claro que:

$$
a<q(a)<q(\tilde{a})<\tilde{a}<b<q(b)<q(y)<y .
$$

Além disso, o sinal de $q(x)-x$ troca pelo menos três vezes entre $a$ e $y$. Portanto, $q$ possui pelo menos três pontos fixos.

- Caso $(-,-,+), \operatorname{com} \tilde{b}<b$ :

Afirmação. Existem $q \in G$ e $y_{1}, y_{2} \in \mathbb{R}$, tais que:

$$
q\left(y_{1}\right)<y_{1}<a<q(a)<q(b)<b<y_{2}<q\left(y_{2}\right) .
$$

Portanto, $q$ possui pelo menos três pontos fixos.

Prova. Tome novamente $f=h \circ g \circ h^{-1} \in G$. Note que pelo Lema 5.3.4 $g^{n} \in G$ e é do tipo $(-,-,+)$ com pontos fixos $\{a, b\}$, para todo $n \in \mathbb{N}$. Além disso,

$$
\lim _{n \rightarrow+\infty} g^{n}(x)=-\infty \text {, para todo } x \in(-\infty, a)
$$

Assim, fixando $y_{1}<a$, existe $n_{1} \in \mathbb{N}$, tal que: $g^{n}\left(y_{1}\right)<f\left(y_{1}\right)$, para todo $n \geq n_{1}$. E também temos que

$$
\lim _{n \rightarrow+\infty} g^{n}(x)=+\infty, \text { para todo } x \in(b, \infty)
$$

Assim, fixando $y_{2}>b$, existe $n_{2} \in \mathbb{N}$, tal que: $g^{n}\left(y_{2}\right)>f\left(y_{2}\right)$, para todo $n \geq n_{2}$. Defina então, $q:=f^{-1} \circ g^{n_{0}} \in G$, onde $n_{0}=\max \left\{n_{1}, n_{2}\right\}$.

Como $g^{n_{0}}$ é do tipo $(-,-,+)$, temos que $g^{n_{0}}(x)<x$ para todo $x<a$ e $g^{n_{0}}(x)>x$ para todo $x>b$. E como $f$ também é do tipo $(-,-,+)$, temos que $f(x)<x$ para todo $x<\tilde{a}$ e $f(x)>x$ para todo $x>\tilde{b}$. Em particular, $f(a)<a$ e $f(b)>b$. Esboço: 


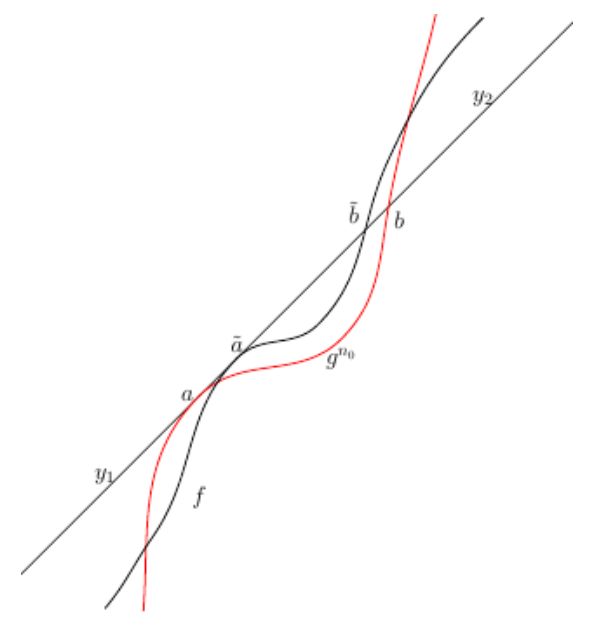

De forma mais precisa, temos as seguintes desigualdades:

$$
\begin{gathered}
g^{n_{0}}\left(y_{1}\right)<f\left(y_{1}\right) \Rightarrow f^{-1} \circ g^{n_{0}}\left(y_{1}\right)<y_{1} \Rightarrow q\left(y_{1}\right)<y_{1}, \\
g^{n_{0}}(a)=a>f(a) \Rightarrow f^{-1} \circ g^{n_{0}}(a)>a \Rightarrow q(a)>a, \\
g^{n_{0}}(b)=b<f(b) \Rightarrow f^{-1} \circ g^{n_{0}}(b)<b \Rightarrow q(b)<b, \\
g^{n_{0}}\left(y_{2}\right)>f\left(y_{2}\right) \Rightarrow f^{-1} \circ g^{n_{0}}\left(y_{2}\right)>y_{2} \Rightarrow q\left(y_{2}\right)>y_{2} .
\end{gathered}
$$

Por fim, lembre-se que $a<b$, e que escolhemos $y_{1}<a$ e $y_{2}>b$. Portanto: $y_{1}<a<b<y_{2}$. Assim, usando o fato de que $q$ é crescente e as quatro desigualdades acima, fica claro que:

$$
q\left(y_{1}\right)<y_{1}<a<q(a)<q(b)<b<y_{2}<q\left(y_{2}\right) .
$$

Além disso, o sinal de $q(x)-x$ troca pelo menos três vezes entre $y_{1}$ e $y_{2}$. Portanto, $q$ possui pelo menos três pontos fixos.

Concluímos assim a prova da Proposição 5.2.4 no caso $(-,-,+)$.

\subsection{2}

\section{Prova da Proposição 5.2.4 no caso $(+,-,+)$}

Este último caso demanda uma construção diferente das anteriores, e muito mais trabalhosa. Por isso, apresentaremos dois lemas que vão simplificar um pouco o nosso trabalho.

Lema 5.3.10 Se $g \in G$ do tipo $(+,-,+)$ com pontos fixos $\{a, b\}$, então

$$
\text { G. } a \cap(a, b) \neq \emptyset \Rightarrow \text { existe } h \in G \text { tal que } h(a) \in(a, b) \text { e } h(b)<b \text {. }
$$

Prova. Se $G . a \cap(a, b) \neq \emptyset$, então existe $p \in G \operatorname{com} p(a) \in(a, b)$. Sabemos que $p(b) \neq b$ pelo Lema 5.3.2. Temos então, dois casos: ou $p(b)<b$, ou $p(b)>b$. 
No primeiro caso, basta tomar $h:=p$, que concluí a prova do lema.

Para o segundo caso, defina

$$
h:=p \circ g \circ p^{-1},
$$

dessa forma $h$ tem pontos fixos $\{p(a), p(b)\}$ e é do tipo $(+,-,+)$, pelo Lema 5.3.7. Assim, $h(x)>x$, para todo $x \in(-\infty, p(a))$ e $h(x)<x$, para todo $x \in(p(a), p(b))$. Como $p(a) \in(a, b)$ e $p(b)>b$, temos:

$$
a<p(a)<b<p(b) \Rightarrow a \in(-\infty, p(a)) \text { e } b \in(p(a), p(b)) .
$$

Portanto, $h(a)>a$ e $h(b)<b$. Como $h$ é crescente temos $h(a)<h(b)$ e portanto:

$$
a<h(a)<h(b)<b \Rightarrow h(a) \in(a, b) \text { e } h(b)<b
$$

como desejado.

Lema 5.3.11 Seja $g \in G$ do tipo $(+,-,+)$, com pontos fixos $\{a, b\}$. Se existe $h \in G \operatorname{com} h(a), h(b) \in(a, b)$, então existem $u, v \in G \operatorname{com} u(b)<a$ e $v(a)>b$.

Prova. Defina $f=h \circ g^{-1} \circ h^{-1}$. Assim pelos Lemas 5.3.5 e 5.3.7, $f$ é do tipo $(-,+,-)$ com pontos fixos $\{h(a), h(b)\} \in(a, b)$. Portanto:

$$
\begin{gathered}
a<h(a) \Rightarrow f(a)<a<h(a), \\
h(b)<b \Rightarrow h(b)<f(b)<b,
\end{gathered}
$$

e temos assim $f(a)<a<f(b)<b$. Agora, definimos $t=f \circ g \circ f^{-1}$. Assim, pelo Lema 5.3.7, $t$ é do tipo $(+,-,+)$ com pontos fixos $\{f(a), f(b)\}$. Portanto,

$$
\begin{gathered}
a \in(f(a), f(b)) \Rightarrow t(a)<a, \\
b \in(f(b),+\infty) \Rightarrow t(b)>b,
\end{gathered}
$$

e temos $t(a)<a<b<t(b)$.

Por fim, definimos $s=t \circ g \circ t^{-1}$ que é do tipo $(+,-,+)$ com pontos fixos $\{t(a), t(b)\}$. Assim, para todo $x \in(t(a), t(b))$,

$$
\lim _{n \rightarrow+\infty} s^{n}(x)=t(a) \text { e } \lim _{n \rightarrow+\infty} s^{-n}(x)=t(b) .
$$

Como $t(a)<a$ e $b \in(t(a), t(b))$ :

$\exists n_{1} \in \mathbb{N}$ tal que $s^{n_{1}}(b)<a$. 
Definimos então $u=s^{n_{1}}$, e obtemos $u(b)<a$.

Agora, como $t(b)>b$ e $a \in(t(a), t(b))$ :

$$
\exists n_{2} \in \mathbb{N} \text { tal que } s^{-n_{2}}(a)>b \text {. }
$$

Definimos então $v=s^{-n_{2}}$, e obtemos $v(a)>b$.

Agora, estamos prontos para concluir a prova da Proposição 5.2.4 no caso $(+,-,+)$.

Prova. Seja $g \in G$ do tipo $(+,-,+)$, com pontos fixos $\{a, b\}$. Suponha por absurdo que:

$$
G . a \cap(a, b) \neq \emptyset .
$$

Portanto, pelo Lema 5.3.10, existe $h \in G$, tal que $h(a), h(b) \in(a, b)$. Defina portanto $\tilde{a}:=h(a)$ e $\tilde{b}:=h(b)$. Pelo Lema 5.3 .11 existem $u, v \in G$, tais que $u(b)<a$ e $b<v(a)$.

Afirmação. Existem $q \in G$, e $y_{1}, y_{2} \in \mathbb{R}$, tais que:

$$
q\left(y_{1}\right)<y_{1}<a<q(a)<q(b)<b<y_{2}<q\left(y_{2}\right)
$$

Portanto, $q$ possui pelo menos três pontos fixos.

Prova. Definimos os seguintes elementos de $G$ :

- $f=h \circ g \circ h^{-1}$ do tipo $(+,-,+)$, com pontos fixos $\{h(a), h(b)\}=\{\tilde{a}, \tilde{b}\}$,

$-h_{u}=u \circ g \circ u^{-1}$ do tipo $(+,-,+)$ e pontos fixos $\{u(a), u(b)\}$,

$-h_{v}=v \circ g \circ v^{-1}$ do tipo $(+,-,+)$ e pontos fixos $\{v(a), v(b)\}$.

Lembre-se que os pontos fixos estão ordenados da seguinte forma:

$$
u(a)<u(b)<a<\tilde{a}<\tilde{b}<b<v(a)<v(b) .
$$

Seja,

$$
s_{n}=f^{n} \circ h_{v} \circ f^{-n} .
$$

Note que para todo $x \in(-\infty, \tilde{a}), \lim _{n \rightarrow+\infty} f^{-n}(x)=-\infty$ e portanto existe $n_{1} \in \mathbb{N}$, tal que:

$$
f^{-n}(a)<h_{v}^{-1}(a), \text { para todo } n \geq n_{1} .
$$

Portanto, como ã é ponto fixo de $f^{n}$, temos:

$$
\begin{gathered}
s_{n}(a)=f^{n} \circ h_{v} \circ f^{-n}(a)<f^{n} \circ h_{v}\left(h_{v}^{-1}(a)\right)=f^{n}(a)<\tilde{a} \\
\Rightarrow s_{n}(a)<\tilde{a}, \text { para todo } n \geq n_{1}
\end{gathered}
$$


Note também que para todo $x \in(\tilde{b},+\infty), \lim _{n \rightarrow+\infty} f^{n}(x)=+\infty$ e como $\tilde{b}<v(a)$, segue que $h_{v}(\tilde{b})>\tilde{b}$ e portanto existe $n_{2} \in \mathbb{N}$, tal que:

$$
f^{n}\left(h_{v}(\tilde{b})\right)>b+2, \text { para todo } n \geq n_{2}
$$

E lembre que $b>\tilde{b}$. Assim:

$$
\begin{gathered}
s_{n}(b)=f^{n} \circ h_{v} \circ f^{-n}(b)>f^{n} \circ h_{v} \circ f^{-n}(\tilde{b})=f^{n} \circ h_{v}(\tilde{b})>b+2 \\
\Rightarrow s_{n}(b)>b+2, \text { para todo } n \geq n_{2} .
\end{gathered}
$$

Definimos então

$$
s:=s_{\tilde{n}} \text { onde, } \tilde{n}=\max \left\{n_{1}, n_{2}\right\}
$$

e temos: $s(a)<\tilde{a}$ e $s(b)>b+2$.

Seja também,

$$
t_{m}=g^{-m} \circ h_{u} \circ g^{m} .
$$

Note que para todo $x \in(a,+\infty), \lim _{m \rightarrow+\infty} g^{-m}(x)=b>\tilde{b}$ e como $u(b)<a$, segue que $h_{u}(a)>a$ e portanto existe $m_{1} \in \mathbb{N}$, tal que:

$$
g^{-m}\left(h_{u}(a)\right)>\tilde{b}, \text { para todo } m \geq m_{1} .
$$

Portanto, como a é ponto fixo de $g^{m}$, temos:

$$
\begin{gathered}
t_{m}(a)=g^{-m} \circ h_{u} \circ g^{m}(a)=g^{-m} \circ h_{u}(a)>\tilde{b} \\
\Rightarrow t_{m}(a)>\tilde{b}, \text { para todo } m \geq m_{1} .
\end{gathered}
$$

Note também que para todo $x \in(b,+\infty), \lim _{m \rightarrow+\infty} g^{-m}(x)=b<b+1$ e como $u(b)<b$, segue que $h_{u}(b)>b$ e portanto existe $m_{2} \in \mathbb{N}$, tal que:

$$
g^{-m}\left(h_{u}(b)\right)<b+1, \text { para todo } m \geq m_{2}
$$

E lembre que, $b$ é ponto fixo de $g^{m}$. Assim:

$$
\begin{gathered}
t_{n}(b)=g^{-m} \circ h_{u} \circ g^{m}(b)=g^{-m} \circ h_{u}(b)<b+1 \\
\Rightarrow t_{m}(b)<b+1, \text { para todo } m \geq m_{2} .
\end{gathered}
$$

Definimos então

$$
t:=t_{\tilde{m}} \text { onde, } \tilde{m}=\max \left\{m_{1}, m_{2}\right\}
$$

e temos: $t(a)>\tilde{b}$ e $t(b)<b+1$. 
Lembramos que

$$
\begin{gathered}
\operatorname{Fix}(s)=f^{\tilde{n}}\left(\operatorname{Fix}\left(h_{v}\right)\right)=\left\{f^{\tilde{n}}(v(a)), f^{\tilde{n}}(v(b))\right\} \\
\operatorname{Fix}(t)=g^{-\tilde{m}}\left(\operatorname{Fix}\left(h_{u}\right)\right)=\left\{g^{-\tilde{m}}(u(a)), g^{-\tilde{m}}(u(b))\right\}
\end{gathered}
$$

com

$$
g^{-\tilde{m}}(u(a))<g^{-\tilde{m}}(u(b))<u(b)<a<b<v(a)<f^{\tilde{n}}(v(a))<f^{\tilde{n}}(v(b)) .
$$

Defina, $y_{1}:=g^{-\tilde{m}}(u(a))$. Assim, $y_{1}$ é ponto fixo de $t$ e $y_{1}$ é menor que os dois pontos fixos de $s$, que é do tipo $(+,-,+)$. Portanto: $t\left(y_{1}\right)=y_{1}$ e $s\left(y_{1}\right)>y_{1}$.

Defina $y_{2}:=f^{\tilde{n}}(v(b))$. Assim, $y_{2}$ é ponto fixo de $s$, e $y_{2}$ é maior que os dois pontos fixos de $t$, que é do tipo $(+,-,+)$. Portanto: $s\left(y_{2}\right)=y_{2}$ e $t\left(y_{2}\right)>y_{2}$.

Por fim, defina $q:=s^{-1} \circ t \in G$. Segue o esboço de $s$ e $t$ :

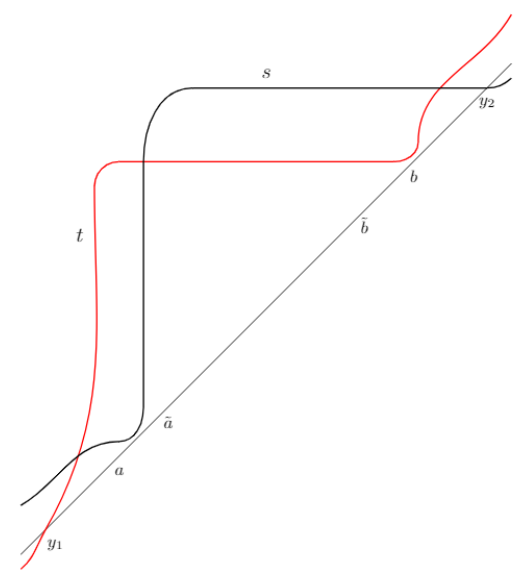

E temos as seguintes desigualdades:

$$
\begin{gathered}
t\left(y_{1}\right)=y_{1}<s\left(y_{1}\right) \Rightarrow s^{-1} \circ t\left(y_{1}\right)<y_{1} \Rightarrow q\left(y_{1}\right)<y_{1}, \\
t(a)>\tilde{b}>\tilde{a}>s(a) \Rightarrow s^{-1} \circ t(a)>a \Rightarrow q(a)>a, \\
t(b)<b+1<b+2<s(b) \Rightarrow s^{-1} \circ t(b)<b \Rightarrow q(b)<b, \\
t\left(y_{2}\right)>y_{2}=s\left(y_{2}\right) \Rightarrow s^{-1} \circ t\left(y_{2}\right)>y_{2} \Rightarrow q\left(y_{2}\right)>y_{2} .
\end{gathered}
$$

Por fim, basta lembrar que $a<b$ e que escolhemos $y_{1}<a$ e $y_{2}>b$, portanto: $y_{1}<a<b<y_{2}$. Assim, usando o fato de que $q$ é crescente e as quatro desigualdades acima, fica claro que:

$$
q\left(y_{1}\right)<y_{1}<a<q(a)<q(b)<b<y_{2}<q\left(y_{2}\right)
$$


Além disso, o sinal de $q(x)-x$ troca pelo menos três vezes entre $y_{1}$ e $y_{2}$. Portanto, $q$ possui pelo menos três pontos fixos. Concluindo a afirmação.

E portanto, temos uma contradição com o fato de $q \in G$ ser não-trivial. Concluímos assim a prova da Proposição 5.2.4 no caso $(+,-,+)$. 


\section{Referências bibliográficas}

[Bo] BONATTI, C. Groupes et dynamiques. 2016.

[Ho] HÖLDER, O. Die axiome der quantität und die lehre vom mass. ber. verh. sachs. ges. wiss. Leipzig, Math. Phys., C1. 53:1-64, 1901.

[Na] NAVAS, A. Groups of circle diffeomorphisms. Chicago Lectures in Mathematics, University of Chicago Press, 2011. 